\title{
UNIFORM CONFIDENCE BANDS FOR NONPARAMETRIC ERRORS-IN-VARIABLES REGRESSION
}

\author{
KENGO KATO AND YUYA SASAKI
}

\begin{abstract}
This paper develops a method to construct uniform confidence bands for a nonparametric regression function where a predictor variable is subject to a measurement error. We allow for the distribution of the measurement error to be unknown, but assume the availability of validation data or repeated measurements on the latent predictor variable. The proposed confidence band builds on the deconvolution kernel estimation and a novel application of the multiplier bootstrap method. We establish asymptotic validity of the proposed confidence band. To our knowledge, this is the first paper to derive asymptotically valid uniform confidence bands for nonparametric errors-in-variables regression.
\end{abstract}

\section{INTRODUCTION}

Consider the nonparametric errors-in-variables (EIV) regression model with classical measurement error

$$
Y=g(X)+\epsilon, W=X+U, \mathrm{E}[\epsilon \mid X, U]=0,
$$

where each of $Y, X, \epsilon, W$, and $U$ is a univariate random variable, and $U$ is independent from $X$. We observe $(Y, W)$, but observe neither $X$ nor $U \mathbb{1}^{1}$ Furthermore, we assume that the distribution of $U$ is unknown. The variable $X$ is a latent predictor variable, while $U$ is a measurement error. Of interest are estimation of and inference on the regression function $g(x)=\mathrm{E}[Y \mid X=x]$. In particular, we are interested in constructing uniform confidence bands for $g$. Confidence bands provide a simple graphical description of the extent to which a nonparametric estimator varies at design points, thereby quantifying uncertainties of the nonparametric estimator. However, construction of confidence bands tends to be challenging, especially for complex nonparametric models (we refer to Wasserman (2006); Hall and Horowitz (2013); Giné and Nickl (2016) as general references on confidence bands in nonparametric statistical models).

Indeed, despite the rich literature on consistent estimation of nonparametric EIV regression, the literature on pointwise or uniform confidence bands for nonparametric EIV regression is limited see below for a literature review - likely because of its complexity. Even pointwise inference on $g$ under the assumption that the measurement error distribution is known is considered by experts

Date: First arXiv version: February 11, 2017. This version: June 17, 2019.

Key words and phrases. confidence band, deconvolution, errors-in-variables regression, multiplier bootstrap.

JEL Code: C14.

${ }^{1}$ Although we state the independence and mean independence restrictions as a concise sufficient condition for the identification, we remark that they may be relaxed as in Schennach (2013). Specifically, it suffices to assume that $\mathrm{E}\left[e^{i t(X+U)}\right]=\mathrm{E}\left[e^{i t X}\right] \mathrm{E}\left[e^{i t U}\right]$ and $\mathrm{E}\left[g(X) e^{i t(X+U)}\right]=\mathrm{E}\left[g(X) e^{i t X}\right] \mathrm{E}\left[e^{i t U}\right]$ for all $t \in \mathbb{R}$. 
to be difficult..$^{2}$ This is because, as discussed in Delaigle et al. (2015): 1) the asymptotic variance of the "deconvolution kernel" estimator of $g$ is non-trivial to estimate and so inference based on limiting distributions is difficult to implement; and 2) it is not straightforward to devise a way to implement bootstrap for inference on $g$ due to the unobservability of $X$ in data.

With all these challenges recognized in the literature, the present paper attempts to solve an even more challenging problem of constructing uniform confidence bands for the regression function $g$ without assuming that the measurement error distribution is known. To deal with unknown measurement error distribution, we assume that there is an independent sample $\left\{\eta_{1}, \ldots, \eta_{m}\right\}$ from the measurement error distribution, in addition to an independent sample $\left\{\left(Y_{1}, W_{1}\right), \ldots,\left(Y_{n}, W_{n}\right)\right\}$ from the distribution of $(Y, W)$, where $m=m_{n} \rightarrow \infty$ as $n \rightarrow \infty$. (The auxiliary sample $\left\{\eta_{1}, \ldots, \eta_{m}\right\}$ need not be independent from $\left\{\left(Y_{1}, W_{1}\right), \ldots,\left(Y_{n}, W_{n}\right)\right\}$.) Real data scenarios of such additional data availability that are plausible in economics, social sciences, and biomedical sciences include: the case where validation data is available for data combination; and the case where repeated measurements on $X$ with errors one of which is symmetrically distributed are available. These patterns of data requirements are often considered in the existing literature with measurement errors that we review below.

Under this setup, we develop a method to construct confidence bands for the regression function g. Our method builds on the deconvolution kernel estimation (Fan and Truong, 1993), and a novel application of the multiplier (or wild) bootstrap method. Bootstrap methods for confidence bands for nonparametric functions have been established previously - see, for example, Hall and Horowitz (2013); Chernozhukov et al. (2014b) that also use Gausssian process approximations. Our construction of the multiplier process differs from the standard approach in the error-free case (cf. Neumann and Polzehl, 1998), and is tailored to EIV regression; see Remark 2.1 ahead. Building on non-trivial applications of the probabilistic techniques developed in Chernozhukov et al. (2014a b, 2016), we establish asymptotic validity of the proposed confidence band, i.e., the proposed confidence band contains the true regression function with probability approaching the nominal coverage probability. In the present paper, as in Bissantz et al. (2007); Schmidt-Hieber et al. (2013); Delaigle et al. (2015) that study inference in deconvolution and EIV regression, we focus for a technical reason on the case where the measurement error density is ordinary smooth, i.e., the characteristic function of the measurement error distribution decays at most polynomially fast in the tail (cf. Fan, 1991a; Fan and Truong, 1993).

In addition to these contributions, we also provide a practical guideline by adapting the regularization method of Delaigle et al. (2008) and the bandwidth selection procedures of Delaigle and Hall (2008); Delaigle et al. (2015) to our framework. We conduct simulation studies to verify the finite sample performance of the proposed confidence band. The simulation studies show that the proposed confidence band, combined with the proposed bandwidth selection rule, works well. Applying our method to a combination of the two data sets, the National Health and Nutrition Examination

\footnotetext{
2 Delaigle et al. (2015), who study pointwise confidence bands for nonparametric EIV regression under the assumption that the measurement error distribution is known, state that "despite their practical importance, to our knowledge confidence bands in nonparametric EIV regression have largely been ignored so far. We show that the problem is particularly complex, much more so than in the standard error-free setting." (Delaigle et al. 2015, p.149)
} 
Survey (NHANES) and the Panel Survey of Income Dynamics (PSID), we draw confidence bands for nonparametric regressions of medical costs on the body mass index (BMI), accounting for measurement errors in BMI. Finally, we discuss extensions of our results to specification testing, cases with additional error-free regressors, and confidence bands for conditional distribution functions.

1.1. Related Literature. In order to locate the present paper in the context of the relevant literature, it is useful to first review measurement error models and deconvolution. We refer to books by Carroll et al. (2006); Meister (2009); Horowitz (2009) and surveys by Chen et al. (2011) and Schennach (2016) for general references. The genesis of this literature features the deconvolution kernel density estimation with known error distributions (Carroll and Hall, 1988; Stefanski and Carroll, 1990; Fan, 1991a b), followed by that with unknown error distributions (Diggle and Hall, 1993; Horowitz and Markatou, 1996; Neumann, 1997; Efromovich, 1997; Li and Vuong, 1998; Delaigle et al., 2008; Johannes, 2009; Comte and Lacour, 2011). Diggle and Hall (1993); Neumann (1997); Efromovich (1997); Johannes (2009); Comte and Lacour (2011) assume the availability of an auxiliary sample from the measurement error distribution, while Horowitz and Markatou (1996); Delaigle et al. (2008) assume repeated measurements with symmetrically and identically distributed errors. For repeated measurements without symmetry of error distributions, Li and Vuong (1998) propose an alternative density estimator based on Kotlarski's lemma (cf. Kotlarski, 1967) that does not require known error distribution; see also Bohnomme and Robin (2010); Comte and Kappus (2015) for further developments. More recently, Delaigle and Hall (2016) propose a new approach of non-parametric deconvolution when the error distribution is unknown and there is no additional data available. Methods to construct confidence bands in deconvolution are developed by Bissantz et al. (2007); Bissantz and Holzmann (2008); van Es and Gugushvili (2008); Lounici and Nickl (2011); Schmidt-Hieber et al. (2013) for the case of known error distribution, and more recently by Kato and Sasaki (2016) for the case of unknown error distribution. See also Adusumilli et al. (2016) for uniform confidence bands for the distribution function in a deconvolution problem.

Similarly to the density estimation, the literature on nonparametric EIV regression estimation often takes the deconvolution kernel approach. Fan and Truong (1993) propose to substitute the deconvolution kernel in the Nadaraya-Watson estimator - also see Fan and Masry (1992) for pointwise asymptotic normality, Delaigle and Meister (2007) for extensions to heteroscedastic measurement errors, Delaigle et al. (2009) for local polynomial extensions, and Delaigle et al. (2015) for pointwise inference. Delaigle et al. (2008) estimate the error characteristic function using repeated measurements on $X$ with symmetrically and identically distributed errors, and substitute the estimated error characteristic function into the deconvolution kernel. Li (2002) and Schennach (2004) also work with cases with repeated measurements but without assuming symmetry of error distributions, and propose alternative approaches to estimate the regression function based on Kotlarski's lemma. See also Carroll et al. (1999); Schennach et al. (2012); Schennach and Hu $(2013) ; \mathrm{Hu}$ and Sasaki (2015).

Our method of inference is based on the deconvolution kernel estimation. We mainly focus on (i) the case where a sample drawn from the error distribution is available; (ii) the case where validation data is available for data combination; and (iii) the case where repeated measurements 
with errors one of which is symmetrically distributed are available. For (ii) data combination with validation data, our model shares similarities albeit different assumptions to that of the nonparametric instrumental variables (NPIV) regression, for which Horowitz and Lee (2012); Chen and Christensen (2018), and Babii (2016) develop methods to construct confidence bands as we do for nonparametric EIV regression.

We note the following two references as particularly relevant benchmarks for identifying our contributions. One reference is Schennach (2004) that derives pointwise asymptotic normality for the nonparametric EIV regression estimator different from ours, under unknown error distribution. To this existing result, our contributions are four-fold. First, we provide a method of uniform inference as opposed to a pointwise one. Second, we propose a method of bandwidth selection for valid inference. Third, while the existing result left aside the issue of variance estimation and thus are not readily applicable in practice, we provide a bootstrap method for ease of practical implementation. Fourth, we devise lower-level assumptions which are easier to verify with concrete examples of distribution and conditional moment functions. The other reference is Delaigle et al. (2015) that suggests a method of pointwise inference via bootstrap for nonparametric EIV regression with known error distribution. To this existing result, our contributions are three-fold. First, our method allows for unknown error distribution. Second, we provide a method of uniform inference as opposed to a pointwise one. Third, we provide formal theories to support the asymptotic validity of our bootstrap method. Delaigle et al. (2015) mention how to modify their methodology to the case where the measurement error distribution is unknown, and to construct uniform confidence bands. However, their theoretical results do not formally cover those cases.

Finally, Birke et al. (2010) and Proksh et al. (2015) obtain confidence bands for inverse regression with fixed equidistant designs (the fixed equidistant design assumption is substantial in their setups and analyses); the inverse regression is related to but different from our EIV regression, and our setup does not allow fixed equidistant designs because of measurement errors. The methodologies and the proof strategies are also different; e.g. both of those papers rely on Gumbel approximations for validity of the confidence bands, which we do not.

Importantly, to the best of our knowledge, none of the existing results covers uniform confidence bands for EIV regression (1), even under the simpler setting that the measurement error distribution is known. The present paper fills this important void.

1.2. Notations and Organization. For a non-empty set $T$ and a (complex-valued) function $f$ on $T$, we use the notation $\|f\|_{T}=\sup _{t \in T}|f(t)|$. Let $\ell^{\infty}(T)$ denote the Banach space of all bounded real-valued functions on $T$ with norm $\|\cdot\|_{T}$. The convolution of $f_{X}$ and $f_{U}$ is denoted and defined by $\left(f_{X} * f_{U}\right)(y)=\int_{\mathbb{R}} f_{X}(x) f_{U}(y-x) d x$. The Fourier transform of an integrable function $f$ on $\mathbb{R}$ is defined by $\varphi_{f}(t)=\int_{\mathbb{R}} e^{i t x} f(x) d x, t \in \mathbb{R}$, where $i=\sqrt{-1}$ denotes the imaginary unit throughout the paper. We refer to Folland (1999) as a basic reference on Fourier analysis. For any positive sequences $a_{n}$ and $b_{n}$, we write $a_{n} \sim b_{n}$ if $a_{n} / b_{n}$ is bounded and bounded away from zero. For any $a, b \in \mathbb{R}$, let $a \wedge b=\min \{a, b\}$ and $a \vee b=\max \{a, b\}$. For $a \in \mathbb{R}, b>0$, we use the shorthand notation $[a \pm b]=[a-b, a+b]$. Let $\stackrel{d}{=}$ denote the equality in distribution. 
The rest of the paper is organized as follows. In Section 2, we informally present our methodology to construct uniform confidence bands for $g$. In Section 3, we present asymptotic validity of the proposed confidence band under suitable regularity conditions. In Section 4 , we propose a practical method to choose the bandwidth. In Section 5, we conduct simulation studies to verify the finite sample performance of the proposed confidence band. In Section 6, we apply the proposed method to a combination of two empirical data sets. In Section 7, we discuss extensions of our results to specification testing of the conditional mean function, cases with additional regressors without measurement errors, and construction of confidence bands for the conditional distribution function. Section 8 concludes. Appendix contains all the proofs, additional simulation results, and additional details of real data analysis.

\section{Methodology}

In this section, we informally present our methodology to construct confidence bands for $g$. The formal analysis of our confidence bands will be carried out in the next section. We will also discuss some examples of situations where an auxiliary sample from the measurement error distribution is available.

2.1. Deconvolution kernel estimation. We first introduce a deconvolution kernel method to estimate $f_{X}$ and $g$ under the assumption that the distribution of $U$ is known. Let $\left\{\left(Y_{1}, W_{1}\right), \ldots,\left(Y_{n}, W_{n}\right)\right\}$ be an independent sample from the distribution of $(Y, W)$. Throughout the paper, we assume that the densities of $X$ and $U$ exist and are denoted by $f_{X}$ and $f_{U}$, respectively. Let $\varphi_{W}, \varphi_{X}$, and $\varphi_{U}$ denote the characteristic functions of $W, X$, and $U$, respectively. Let $K: \mathbb{R} \rightarrow \mathbb{R}$ be a kernel function such that $K$ is integrable on $\mathbb{R}, \int_{\mathbb{R}} K(x) d x=1$, and its Fourier transform $\varphi_{K}$ is supported in $[-1,1]$ (i.e., $\varphi_{K}(t)=0$ for all $|t|>1$ ).

When $f_{U}$ is known, the deconvolution kernel density estimator of $f_{X}$ reads

$$
\widehat{f}_{X}^{*}(x)=\frac{1}{2 \pi} \int_{\mathbb{R}} e^{-i t x} \widehat{\varphi}_{W}(t) \frac{\varphi_{K}\left(t h_{n}\right)}{\varphi_{U}(t)} d t=\frac{1}{n h_{n}} \sum_{j=1}^{n} K_{n}\left(\left(x-W_{j}\right) / h_{n}\right),
$$

where the function $K_{n}$, called the deconvolution kernel, is defined by

$$
K_{n}(x)=\frac{1}{2 \pi} \int_{\mathbb{R}} e^{-i t x} \frac{\varphi_{K}(t)}{\varphi_{U}\left(t / h_{n}\right)} d t .
$$

See Carroll and Hall (1988); Stefanski and Carroll (1990); Fan (1991a b) for asymptotic properties.

Analogously, Fan and Truong (1993) propose to estimate the regression function $g(x)$ by $\widehat{g}^{*}(x)=$ $\widehat{\mu}^{*}(x) / \widehat{f}_{X}^{*}(x)$, where

$$
\begin{aligned}
\widehat{\mu}^{*}(x) & =\frac{1}{2 \pi} \int_{\mathbb{R}} e^{-i t x}\left(n^{-1} \sum_{j=1}^{n} Y_{j} e^{i t W_{j}}\right) \frac{\varphi_{K}\left(t h_{n}\right)}{\varphi_{U}(t)} d t \\
& =\frac{1}{n h_{n}} \sum_{j=1}^{n} Y_{j} K_{n}\left(\left(x-W_{j}\right) / h_{n}\right) .
\end{aligned}
$$

For asymptotic properties, see Fan and Truong (1993); Fan and Masry (1992), among others.$^{3}$

\footnotetext{
${ }^{3}$ It is worth pointing out that estimation of $f_{X}$ and $g f_{X}$ corresponds to solving certain Fredholm integral equations of the first kind, and therefore estimation of $f_{X}$ and $g f_{X}$ (or $g$ ) is a statistical ill-posed inverse problem. In fact, $f_{X}$
} 
The discussion so far has presumed that the distribution of $U$ is known. In the present paper, we consider the case of unknown distribution of $U$, and assume that there is an independent sample $\left\{\eta_{1}, \ldots, \eta_{m}\right\}$ from the distribution of $U$, i.e., $\eta_{1}, \ldots, \eta_{m} \sim f_{U}$ i.i.d. where $m=m_{n} \rightarrow \infty$ as $n \rightarrow \infty$. We do not assume that $\eta_{1}, \ldots, \eta_{m}$ are independent from $\left\{\left(Y_{1}, W_{1}\right), \ldots,\left(Y_{n}, W_{n}\right)\right\}$. In Section 2.4 . we will discuss examples where such an auxiliary sample from the measurement error distribution is available. Given $\left\{\eta_{1}, \ldots, \eta_{m}\right\}$, we may estimate $\varphi_{U}$ by the empirical characteristic function, namely,

$$
\widehat{\varphi}_{U}(t)=\frac{1}{m} \sum_{j=1}^{m} e^{i t \eta_{j}}
$$

and estimate the deconvolution kernel $K_{n}$ by the plug-in method:

$$
\widehat{K}_{n}(x)=\frac{1}{2 \pi} \int_{\mathbb{R}} e^{-i t x} \frac{\varphi_{K}(t)}{\widehat{\varphi}_{U}\left(t / h_{n}\right)} d t .
$$

We can thus estimate $g(x)$ by $\widehat{g}(x)=\widehat{\mu}(x) / \widehat{f}_{X}(x)$, where

$$
\widehat{\mu}(x)=\frac{1}{n h_{n}} \sum_{j=1}^{n} Y_{j} \widehat{K}_{n}\left(\left(x-W_{j}\right) / h_{n}\right), \quad \widehat{f}_{X}(x)=\frac{1}{n h_{n}} \sum_{j=1}^{n} \widehat{K}_{n}\left(\left(x-W_{j}\right) / h_{n}\right) .
$$

Density estimators of the form $\widehat{f}_{X}$ are studied in Diggle and Hall (1993); Neumann (1997); Efromovich (1997), among others, and nonparametric regression estimators of the form $\widehat{g}$ are studied in Delaigle et al. (2008), among others.

2.2. Motivation for our approach. In this section, we motivate our approach based on the multiplier bootstrap and Gaussian approximation. To this end, we first introduce the multiplier bootstrap in a simple setting, and present intuition for how and why it works. Suppose that an estimator $\widehat{g}(x)$ of $g(x)$ has the influence function representation $\sqrt{n}(\widehat{g}(x)-g(x)) \approx n^{-1 / 2} \sum_{j=1}^{n} \phi_{j}(x)$, and the right-hand side is approximately normal $N\left(0, \sigma^{2}(x)\right)$. In this simple setting, we can construct a confidence interval for $g(x)$ by $\left[\widehat{g}(x) \pm \widehat{\sigma}(x) \Phi^{-1}((1-\tau) / 2)\right]$, where $\Phi$ denotes the distribution function of $N(0,1)$, and $\widehat{\sigma}(x)$ is an estimator of $\sigma(x)$. The multiplier bootstrap can be used to obtain an estimator of the approximating Gaussian distribution $N\left(0, \sigma^{2}(x)\right)$. Specifically, generate multiplier variables $\xi_{1}, \ldots, \xi_{n} \sim N(0,1)$ i.i.d. independently of the data, and consider $Z_{n}^{\xi}(x)=n^{-1 / 2} \sum_{j=1}^{n} \xi_{j} \phi_{j}(x)$. One can generate multiplier variables many times to approximate the distribution of $Z_{n}^{\xi}(x)$ conditionally on the data, which estimates the $N\left(0, \sigma^{2}(x)\right)$ distribution. This method works intuitively because $n^{-1 / 2} \sum_{j=1}^{n} \xi_{j} \phi_{j}(x)$, as a sum of normal random variables, is normal conditionally on $\phi_{1}(x), \ldots, \phi_{n}(x)$ with variance approximately $\sigma^{2}(x)$.

The above idea of the multiplier bootstrap extends to estimating the distribution of $\|\sqrt{n}(\widehat{g}(\cdot)-g(\cdot))\|_{I}$ for a compact interval $I$ of $\mathbb{R}$, where $\|f\|_{I}=\sup _{x \in I}|f(x)|$. Suppose that $\sqrt{n}(\widehat{g}(x)-g(x)) \approx$ $n^{-1 / 2} \sum_{j=1}^{n} \phi_{j}(x)$ for all $x \in I$, and the process $I \ni x \mapsto n^{-1 / 2} \sum_{j=1}^{n} \phi_{j}(x)$ is approximately Gaussian. One can generate $\xi_{1}, \ldots, \xi_{n} \sim N(0,1)$ many times to approximate the distribution of $\left\|Z_{n}^{\xi}\right\|_{I}$ conditionally on the data, which can be used as an estimator for the distribution of

and $g f_{X}$ satisfy $f_{X} * f_{U}=f_{W}$ and $\left(g f_{X}\right) * f_{U}=\mathrm{E}[Y \mid W=\cdot] f_{W}$; these are Fredholm integral equations of the first kind where the right hand side functions are directly estimable (see, e.g., Cavalier (2008) and Horowitz (2009) for an overview of statistical ill-posed inverse problems). 
$\|\sqrt{n}(\widehat{g}(\cdot)-g(\cdot))\|_{I}$. Particularly, the $(1-\tau)$-th quantile of $\left\|Z_{n}^{\xi}(\cdot)\right\|_{I}$ conditionally on the data, denoted by $\widehat{c}_{n}$, can be used to form the uniform confidence band of $g$ on $I$ as $\left[\widehat{g}(x) \pm \widehat{c}_{n}\right], x \in I$ ] We shall recommend and thus present this multiplier bootstrap approach based on the Gaussian approximation, rather than more traditional methods, for the following two reasons. First, the multiplier bootstrap can easily approximate $\widehat{c}_{n}$ by simulating standard normal multipliers, whereas one would otherwise need to compute the covariance function of $I \ni x \mapsto n^{-1 / 2} \sum_{j=1}^{n} \phi_{j}(x)$, which is an infinite-dimensional object, and compute the distribution of the corresponding Gaussian supremum, which is not easy. Second, the traditional approach to construct confidence bands is based on the Gumbel limit distribution for example (cf. Bissantz et al., 2007). Obtaining the Gumbel limit distribution requires additional regularity assumptions than our approach, as discussed in Section 3 in more detail. Furthermore, the convergence to this limit distribution occurs only at a slow log rate Hall (1991). For these reasons, we recommend our approach based on the multiplier bootstrap and Gaussian approximation, and present it in the following section.

2.3. Construction of confidence bands. We now describe our method to construct confidence bands for $g$ based on the estimator $\widehat{g}$. Under the regularity conditions stated below, we will show that $\widehat{g}(x)-g(x)$ can be approximated by $\frac{1}{f_{X}(x) n h_{n}} \sum_{j=1}^{n}\left[\left\{Y_{j}-g(x)\right\} K_{n}\left(\left(x-W_{j}\right) / h_{n}\right)-A_{n}(x)\right]$ uniformly in $x \in I$, where $I$ is a compact interval in $\mathbb{R}$ on which $f_{X}$ is bounded away from zero, and $A_{n}(x)=\mathrm{E}\left[\{Y-g(x)\} K_{n}\left((x-W) / h_{n}\right)\right]$. Let

$$
s_{n}^{2}(x)=\operatorname{Var}\left(\{Y-g(x)\} K_{n}\left((x-W) / h_{n}\right)\right),
$$

and consider the process

$$
\left.\mathrm{Z}_{n}^{*}(x)=\frac{1}{s_{n}(x) \sqrt{n}} \sum_{j=1}^{n}\left[\left\{Y_{j}-g(x)\right\} K_{n}\left(\left(x-W_{j}\right) / h_{n}\right)\right)-A_{n}(x)\right], x \in I,
$$

where $s_{n}(x)=\sqrt{s_{n}^{2}(x)}$. Note that under the regularity conditions stated below, $\inf _{x \in I} s_{n}(x)>0$ for sufficiently large $n$, so that $Z_{n}^{*}$ is well-defined. Furthermore, we will show that there exists a tight Gaussian process $Z_{n}^{G}$ in $\ell^{\infty}(I)$ with mean zero and the same covariance function as $Z_{n}^{*}$, and such that as $n \rightarrow \infty, \sup _{z \in \mathbb{R}}\left|\mathrm{P}\left\{\left\|\mathrm{Z}_{n}^{*}\right\|_{I} \leq z\right\}-\mathrm{P}\left\{\left\|\mathrm{Z}_{n}^{G}\right\|_{I} \leq z\right\}\right| \rightarrow 0$. Recall that $\|f(x)\|_{I}=\sup _{x \in I}|f(x)|$ for a function $f$ on $I$, and $\ell^{\infty}(I)$ is the Banach space of bounded real functions on $I$ equipped with norm $\|\cdot\|_{I}$. This in turn yields that

$$
\sup _{z \in \mathbb{R}}\left|\mathrm{P}\left\{\left\|\widehat{Z}_{n}\right\|_{I} \leq z\right\}-\mathrm{P}\left\{\left\|Z_{n}^{G}\right\|_{I} \leq z\right\}\right| \rightarrow 0,
$$

where $\left\{\widehat{Z}_{n}(x): x \in I\right\}$ is a process defined by

$$
\widehat{\mathrm{Z}}_{n}(x)=f_{X}(x) \sqrt{n} h_{n}(\widehat{g}(x)-g(x)) / s_{n}(x), x \in I .
$$

\footnotetext{
${ }^{4}$ In the current simplified setup for a concise motivation, the width of the band is constant, $\widehat{c}_{n}$, on $I$. We later extend this framework to self-normalized processes, and thence we obtain non-constant widths of the band on $I$.

${ }^{5}$ At first glance, this equation appears to suggest that the rate of convergence is $1 / \sqrt{n} h_{n}$, but the this is not the case - see discussions in Section 3 This equation entailing the scale of $\sqrt{n} h_{n}$ is due to our definition of $Z_{n}^{*}$ for convenience of our proofs.
} 
Therefore, if we denote by $c_{n}^{G}(1-\tau)$ the $(1-\tau)$-quantile of $\left\|Z_{n}^{G}\right\|_{I}$ for $\tau \in(0,1)$, then a band of the form

$$
\widehat{\mathcal{C}}_{1-\tau}^{*}(x)=\left[\widehat{g}(x) \pm \frac{s_{n}(x)}{f_{X}(x) \sqrt{n} h_{n}} c_{n}^{G}(1-\tau)\right], x \in I
$$

will contain $g(x), x \in I$ with probability at least $1-\tau+o(1)$ as $n \rightarrow \infty$. In fact, it holds that $\mathrm{P}\left\{g(x) \in \widehat{\mathcal{C}}_{1-\tau}^{*}(x) \forall x \in I\right\}=\mathrm{P}\left\{\left\|\widehat{\mathbf{Z}}_{n}\right\|_{I} \leq c_{n}^{G}(1-\tau)\right\}=\mathrm{P}\left\{\left\|\mathrm{Z}_{n}^{G}\right\|_{I} \leq c_{n}^{G}(1-\tau)\right\}+o(1) \geq 1-\tau+o(1)$.

In practice, $f_{X}(x), s_{n}^{2}(x)$, and $c_{n}^{G}(1-\tau)$ are all unknown, and we have to estimate them. We estimate $f_{X}(x)$ and $s_{n}^{2}(x)$ by $\widehat{f}_{X}(x)$ and

$$
\widehat{s}_{n}^{2}(x)=\frac{1}{n} \sum_{j=1}^{n}\left\{Y_{j}-\widehat{g}(x)\right\}^{2} \widehat{K}_{n}^{2}\left(\left(x-W_{j}\right) / h_{n}\right),
$$

respectively. Note that $\left(\mathrm{E}\left[A_{n}(x)\right]\right)^{2}$ is negligible relative to $s_{n}^{2}(x)$ so that we have ignored $\left(\mathrm{E}\left[A_{n}(x)\right]\right)^{2}$ in estimation of $s_{n}^{2}(x)$. Note also that $\left.\sum_{j=1}^{n}\left(Y_{j}-\widehat{g}(x)\right) \widehat{K}_{n}\left(\left(x-W_{j}\right) / h_{n}\right)\right)=0$.

Next, we estimate the quantile $c_{n}^{G}(1-\tau)$ by the Gaussian multiplier bootstrap. Generate $\xi_{1}, \ldots, \xi_{n} \sim N(0,1)$ i.i.d., independently of the data $\mathcal{D}_{n}=\left\{Y_{1}, \ldots, Y_{n}, W_{1}, \ldots, W_{n}, \eta_{1}, \ldots, \eta_{m}\right\}$, and consider

$$
\widehat{Z}_{n}^{\xi}(x)=\frac{1}{\widehat{s}_{n}(x) \sqrt{n}} \sum_{j=1}^{n} \xi_{j}\left\{Y_{j}-\widehat{g}(x)\right\} \widehat{K}_{n}\left(\left(x-W_{j}\right) / h_{n}\right),
$$

where $\widehat{s}_{n}(x)=\sqrt{\widehat{s}_{n}^{2}(x)}$. Note that under the regularity conditions stated below, $\inf _{x \in I} \widehat{s}_{n}(x)>0$ with probability approaching one. Conditionally on the data $\mathcal{D}_{n}, \widehat{Z}_{n}^{\xi}$ is a Gaussian process with mean zero and covariance function (presumably) "close" to or "estimates" that of $Z_{n}^{*}$. Hence, we estimate $c_{n}^{G}(1-\tau)$ by $\widehat{c}_{n}(1-\tau)$, defined as the conditional $(1-\tau)$-quantile of $\left\|\widehat{Z}_{n}^{\xi}\right\|_{I}$ given $\mathcal{D}_{n}$, which can be computed via simulations. Now, the resulting confidence band is defined by

$$
\widehat{\mathcal{C}}_{1-\tau}(x)=\left[\widehat{g}(x) \pm \frac{\widehat{s}_{n}(x)}{\widehat{f}_{X}(x) \sqrt{n} h_{n}} \widehat{c}_{n}(1-\tau)\right], x \in I .
$$

Note that, except for the choice of the bandwidth, this confidence band is completely data-driven. We will discuss practical choice of the bandwidth in Section 4 .

Remark 2.1. In the error-free case where we observe $\left\{\left(Y_{j}, X_{j}\right)\right\}_{j=1}^{n}$, the deviation of a traditional kernel regression estimator with kernel $K$ from its mean is approximated by $\frac{1}{f_{X}(x) n h_{n}} \sum_{j=1}^{n} \epsilon_{j} K((x-$ $\left.X_{j}\right) / h_{n}$ ) under suitable regularity conditions. On the other hand, in the EIV case, the deviation of $\widehat{g}(x)$ from its mean is approximated by $\frac{1}{f_{X}(x) n h_{n}} \sum_{j=1}^{n}\left[\left\{Y_{j}-g(x)\right\} K_{n}\left(\left(x-W_{j}\right) / h_{n}\right)\right]$. Note that $\epsilon_{j}=Y_{j}-g\left(X_{j}\right)$ is used in the former error-free case, whereas $Y_{j}-g(x)$ is used in the latter EIV case. Since the conditional variance of $\epsilon_{j}=\left(Y_{j}-g(x)\right)-\left(g(x)-g\left(X_{j}\right)\right)$ given $W_{j}=x$ is not necessarily the same as the the conditional variance of $Y_{j}-g(x)$ given $W_{j}=x$, it is essential to use $Y_{j}-g(x)$ in the EIV case rather than $\epsilon_{j}$ (even if $\epsilon_{j}$ or its estimate were available).

Remark 2.2 (Empirical bootstrap). Instead of the multiplier bootstrap, one can use (a version of) the empirical bootstrap to estimate the quantile $c_{n}^{G}(1-\tau)$, as in Adusumilli et al. (2016) (note: Adusumilli et al. (2016) consider inference on the marginal distribution function of $X$ and 
not consider infererence on $g(x))$. Namely, let $\left(Y_{1}^{*}, W_{1}^{*}\right), \ldots,\left(Y_{n}^{*}, W_{n}^{*}\right)$ be i.i.d. draws from the empirical distribution of $\left\{\left(Y_{1}, W_{1}\right), \ldots,\left(Y_{n}, W_{n}\right)\right\}$, and consider the bootstrap process

$$
\widehat{\mathrm{Z}}_{n}^{E B}(x)=\frac{1}{\widehat{s}_{n}(x) \sqrt{n}} \sum_{i=1}^{n}\left\{Y_{j}^{*}-\widehat{g}(x)\right\} \widehat{K}_{n}\left(\left(x-W_{j}^{*}\right) / h_{n}\right), x \in I .
$$

We note that the expectation of $\left\{Y_{j}^{*}-\widehat{g}(x)\right\} \widehat{K}_{n}\left(\left(x-W_{j}^{*}\right) / h_{n}\right)$ with respect to the bootstrap distribution is $n^{-1} \sum_{j^{\prime}=1}^{n}\left\{Y_{j^{\prime}}-\widehat{g}(x)\right\} \widehat{K}_{n}\left(\left(x-W_{j^{\prime}}\right) / h_{n}\right)=0$ (by the definition of $\left.\widehat{g}(x)\right)$, and so conditionally the right hand side is the sum of independent random variables with mean zero. Then we can estimate $c_{n}^{G}(1-\tau)$ by the conditional $(1-\tau)$-quantile of $\left\|\widehat{Z}_{n}^{E B}\right\|_{I}$ given $\mathcal{D}_{n}$, and so the resulting confidence band is

$$
\widehat{\mathcal{C}}_{1-\tau}^{E B}(x)=\left[\widehat{g}(x) \pm \frac{\widehat{s}_{n}(x)}{\widehat{f}_{X}(x) \sqrt{n} h_{n}} \widehat{c}_{n}^{E B}(1-\tau)\right], x \in I,
$$

which we call the empirical bootstrap confidence band. This empirical bootstrap confidence band is also asymptotically valid under the same conditions as the multiplier bootstrap confidence band. See Appendix A.5 for details.

2.4. Examples. In this section, we present a couple of examples where an auxiliary sample from the measurement error distribution is available.

Example 2.1 (Repeated measurements, Carroll et al. (2006), p.298). Suppose that we observe repeated measurements $\left(W^{(1)}, W^{(2)}\right)$ on $X$ with measurement errors $\left(U^{(1)}, U^{(2)}\right)$, where $W^{(k)}=$ $X+U^{(k)}$ for $k=1,2, X$ and $\left(U^{(1)}, U^{(2)}\right)$ are independent, and the conditional distribution of $U^{(2)}$ given $U^{(1)}$ is symmetric. Note that the unobserved regressor $X$ is common between $k=1,2$. The distribution of $U^{(1)}$ need not be symmetric (in particular, the distributions of $U^{(1)}$ and $U^{(2)}$ may be different), and independence between $U^{(1)}$ and $U^{(2)}$ is not necessary. If we define $W=$ $\left(W^{(1)}+W^{(2)}\right) / 2, U=\left(U^{(1)}+U^{(2)}\right) / 2$, and $\eta=\left(W^{(1)}-W^{(2)}\right) / 2=\left(U^{(1)}-U^{(2)}\right) / 2$, then we have that $W=X+U$ and $U \stackrel{d}{=} \eta$, where $\eta$ is observable.

Note that the constructed sample $\left(\eta_{1}, \ldots, \eta_{n}\right)=\left(\left(W_{1}^{(1)}-W_{1}^{(2)}\right) / 2, \ldots,\left(W_{n}^{(1)}-W_{n}^{(2)}\right) / 2\right)$ behaves as an auxiliary sample of size $m=n$ due to $U \stackrel{d}{=} \eta$. In this way, we transform the repeated measurement framework into the auxiliary sample framework, and therefore our proposed method outlined in Sections 2.1 2.3 for the case of an auxiliary sample directly applies to the case of repeated measurements. This approach differs from the way in which some of preceding papers use repeated measurements (e.g., Delaigle et al., 2008). One implication of this difference is that we can allow for a different set of assumption from that of Delaigle et al. (2008). First, they require conditions on the relative smoothness of $g$ with respect to the measurement error density, while we do not. Second, we only require the conditional distribution of $U^{(2)}$ given $U^{(1)}$ to be symmetric, while they require both $U^{(1)}$ and $U^{(2)}$ to be symmetrically distributed.

For a repeated measurements setup, Schennach (2004) proposes an alternative estimator of $g$ based on Kotlarski's lemma which does not require the symmetry assumption. The form of Schennach's estimator is more complex than ours, and to the best of our knowledge, there is no existing result on asymptotically valid uniform confidence bands for Schennach's estimator. 
It is worth noting that while Schennach's approach can drop the symmetry assumption, it requires another technical assumption that the characteristic function $\varphi_{X}(t)=\mathrm{E}\left[e^{i t X}\right]$ of $X$ does not vanish on the entire real line $\mathbb{R}$. Both Schennach (2004) and we (and in fact most of papers on deconvolution and EIV regression) assume that the characteristic functions of the error variables do not vanish on $\mathbb{R}$, but our approach does allow $\varphi_{X}$ to take zeros. The assumption that $\varphi_{X}$ does not vanish on $\mathbb{R}$ is not innocuous; it is non-trivial to find densities that are compactly supported and have non-vanishing characteristic functions (though these properties are not mutually exclusive; see, e.g., Schennach (2016), Footnote 4), and the assumption excludes densities convolved with distributions whose characteristic functions take zeros, and so on (e.g., convolutions of $k$ uniform densities on $[a, b]$ are piecewise polynomials with degrees $k-1$, and convex combinations of such piecewise polynomials form a rich family of densities, but their characteristic functions take zeros). So, we believe that Schennach's approach and ours are complementary to each other.

Example 2.2 (Data combination). Suppose that we have access to data on $(Y, W)$ and $(W, X)$, separately, but do not have access to data on $(Y, X)$. In this case, we can construct a sample $\left\{Y_{1}, \ldots, Y_{n}, W_{1}, \ldots, W_{n}, \eta_{1}, \ldots, \eta_{m}\right\}$ from a sample of size $n$ on $(Y, W)$ and a sample of size $m$ on $(W, X)$.

This case is often faced by empirical researchers, and various techniques are proposed to combine the two separate samples - see a survey by Ridder and Moffitt (2007). To fix ideas, consider the demand model $Y=g(X)+\epsilon$, where $Y$ denotes the quantity purchased of a product and $X$ denotes the logarithm of its price. Marketing scientists and economists often use Nielsen Homescan data for quantities and prices to analyze this demand model, but the home-scanned prices in this data are subject to imputation errors $U=W-X$. To overcome this issue, Einav et al. (2010) collect data on $(W, X)$ from a large grocery retailer by matching transaction prices $X$ that were recorded by the retailer (at the store) to the prices $W$ recorded by the Homescan panelists. Together with Nielsen Homescan data on $(Y, W)$, Einav et al. suggest to combine the two separate data sets to analyze the demand model.

In the literature, validation data are used as a way to relax the classical measurement error assumption that $X$ and $U$ are independent; see, e.g., Chen et al. (2005). While they allow for nonclassical measurement errors, Chen et al. (2005) focus on the case where the parameter of interest is finite dimensional.

It is worth noting that, when validation data on $(X, W)$ are available, the problem of estimation of $g$ can be considered as a nonparametric instrumental variable (NPIV) problem treating $X$ as an endogenous variable and $W$ as an instrumental variable (see,e.g., Newey and Powell, 2003 , Hall and Horowitz, 2005; Blundell et al., 2007; Chen and Reiss, 2011; Horowitz, 2011, for NPIV models). In fact, observe that $\mathrm{E}[Y \mid W]=\mathrm{E}[g(X) \mid W]$. For NPIV models, Horowitz and Lee (2012) and the more recent paper by Chen and Christensen (2018) develop methods to construct confidence bands for the structural function using series methods, although these papers do not formally consider cases where samples on $(Y, W)$ and $(X, W)$ are different (Babii $(2016)$ also develop methods to construct confidence bands for Tikhonov regularized estimators in NPIV models, but his confidence bands are asymptotically conservative in the sense that the coverage probabilities 
are in general strictly larger than the nominal level even asymptotically). However, we would like to point out that there are difference in underlying assumptions between series estimation of NPIV models and deconvolution kernel estimation in EIV regression. For example, in series estimation of NPIV models, it is often assumed that the distribution of $W$ is compactly supported and the density of $W$ is bounded away from zero on its support (cf. Blundell et al., 2007; Chen and Christensen, 2018). On the other hand, in EIV regression, it is commonly assumed that the characteristic function of the measurement error $U$ is non-vanishing on $\mathbb{R}$ (which leads to identification of the function $g$ ), and in many cases the measurement error $U$ then has unbounded support, which in turn implies that $W$ has unbounded support. Further, while both NPIV and EIV regressions are statistical ill-posed inverse problems, the ways in which the "ill-posedness" is defined are different; in series estimation of NPIV models, the ill-posedness is defined for given basis functions, while in EIV regression, the ill-posedness is defined via how fast the characteristic function of the measurement error distribution decays. Finally, the EIV approach captures all the restrictions implied by the classical measurement error assumption and therefore enjoys more efficiency, while the NPIV approach requires less restrictive assumptions on the measurement error. For these differences and tradeoffs, we believe that our inference results cover different situations from those developed in the NPIV literature.

\section{MAin RESUlts}

In this section, we study asymptotic validity of the proposed confidence band $(6)$. To this end, we make the following assumption. For any given constants $\beta, B>0$, let $\Sigma(\beta, B)$ denote the class of functions $f: \mathbb{R} \rightarrow \mathbb{R}$ such that $f$ is $k$-times differentiable and

$$
\left|f^{(k)}(x)-f^{(k)}(y)\right| \leq B|x-y|^{\beta-k}, \forall x, y \in \mathbb{R},
$$

where $k$ is the integer such that $k<\beta \leq k+1$, and $f^{(k)}$ denotes the $k$-th derivative of $f\left(f^{(0)}=f\right)$. Let $I$ be a compact interval in $\mathbb{R}$.

Assumption 3.1. We assume the following conditions.

(i) $\mathrm{E}\left[Y^{4}\right]<\infty$, the function $w \mapsto \mathrm{E}\left[Y^{2} \mid W=w\right] f_{W}(w)$ is bounded and continuous, and for each $\ell=1,2$, the function $w \mapsto \mathrm{E}\left[|Y|^{2+\ell} \mid W=w\right] f_{W}(w)$ is bounded.

(ii) The functions $\varphi_{X}(t)=\mathrm{E}\left[e^{i t X}\right]$ and $\psi_{X}(t)=\mathrm{E}\left[g(X) e^{i t X}\right]$ for $t \in \mathbb{R}$ are integrable on $\mathbb{R}$.

(iii) The measurement error $U$ has finite mean, $\mathrm{E}[|U|]<\infty$, and its characteristic function, $\varphi_{U}(t)=\mathrm{E}\left[e^{i t U}\right], t \in \mathbb{R}$, does not vanish on $\mathbb{R}$. Furthermore, there exist constants $C_{1}>1$ and $\alpha>0$ such that $C_{1}^{-1}|t|^{-\alpha} \leq\left|\varphi_{U}(t)\right| \leq C_{1}|t|^{-\alpha}$ and $\left|\varphi_{U}^{\prime}(t)\right| \leq C_{1}|t|^{-\alpha-1}$ for all $|t| \geq 1$.

(iv) The functions $f_{X}$ and $g f_{X}$ belong to $\Sigma(\beta, B)$ for some $\beta>1 / 2$ and $B>0$. Let $k$ denote the integer such that $k<\beta \leq k+1$.

(v) Let $K$ be a real-valued integrable function (kernel) on $\mathbb{R}$, not necessarily non-negative, such that $\int_{\mathbb{R}} K(x) d x=1$, and its Fourier transform $\varphi_{K}$ is continuously differentiable and supported in $[-1,1]$. Furthermore, the function $K$ is a $(k+1)$-th order kernel in the sense that $\int_{\mathbb{R}}|x|^{k+1}|K(x)| d x<\infty$ and $\int_{\mathbb{R}} x^{\ell} K(x) d x=0$ for $\ell=1, \ldots, k$

(vi) For all $x \in I, f_{X}(x)>0$ and $\mathrm{E}\left[\{Y-g(x)\}^{2} \mid W=x\right] f_{W}(x)>0$. 
(vii) As $n \rightarrow \infty$,

$$
\frac{\left(\log \left(1 / h_{n}\right)\right)^{2}}{(n \wedge m) h_{n}^{2 \alpha+2}} \rightarrow 0, \frac{n h_{n} \log \left(1 / h_{n}\right)}{m} \rightarrow 0, h_{n}^{\alpha+\beta} \sqrt{n h_{n} \log \left(1 / h_{n}\right)} \rightarrow 0 .
$$

Condition (i) is a moment condition on $Y$, which we believe is not restrictive. Note that, for each $\ell=0,1,2$, if $\mathrm{E}\left[|Y|^{2+\ell} \mid X, U\right]=\mathrm{E}\left[|Y|^{2+\ell} \mid X\right]$, then by comparing the Fourier transforms of both sides, we arrive at the identity

$$
\mathrm{E}\left[|Y|^{2+\ell} \mid W=w\right] f_{W}(w)=\left(\left(\Upsilon_{\ell} f_{X}\right) * f_{U}\right)(w)
$$

where $\Upsilon_{\ell}(x)=\mathrm{E}\left[|Y|^{2+\ell} \mid X=x\right]$ (cf. the proof of Lemma A.4 Case (ii)), and the right hand side is bounded and continuous if $\Upsilon_{\ell} f_{X}$ is bounded (recall that if functions $f_{1}$ and $f_{2}$ on $\mathbb{R}$ are integrable and bounded, respectively, then their convolution $f_{1} * f_{2}$ is bounded and continuous). Note that this does not require $\Upsilon_{\ell}$ to be bounded globally. For Condition (ii), we first note that $\psi_{X}$ is the Fourier transform of $g f_{X}$ (which is integrable by $\mathrm{E}[|Y|]<\infty$ ). Condition (ii) implies that

$$
f_{X} \text { and } g f_{X} \text { are bounded (and continuous), }
$$

which in turn implies that

$$
f_{W}(w)=\int_{\mathbb{R}} f_{X}(w-x) f_{U}(x) d x \text { is bounded and continuous. }
$$

Condition (ii) is satisfied if, e.g., $f_{X}$ and $g f_{X}$ are twice continuously differentiable with integrable derivatives up to the second order; in fact, under such conditions, $\left|\varphi_{X}(t)\right|=o\left(|t|^{-2}\right)$ and $\left|\psi_{X}(t)\right|=$ $o\left(|t|^{-2}\right)$ as $|t| \rightarrow \infty$. However, differentiability of $f_{X}$ and $g f_{X}$ is not strictly necessary for Condition (ii) to hold; e.g., a Laplace density is not differentiable but its Fourier transform is integrable.

Condition (iii) is concerned with the characteristic function of the measurement error. Note that finiteness of the first moment of $U$ ensures that $\varphi_{U}$ is continuously differentiable. In the present paper, as in Bissantz et al. (2007), Schmidt-Hieber et al. (2013), and Delaigle et al. (2015), we assume that the measurement error density is ordinary smooth, namely, $\left|\varphi_{U}(t)\right|$ decays at most polynomially fast as $|t| \rightarrow \infty$ (cf. Fan, 1991a). Informally, the smoother $f_{U}$ is, the faster $\left|\varphi_{U}(t)\right|$ decays as $|t| \rightarrow \infty$, so Condition (iii) restricts smoothness of $f_{U}$. Laplace and Gamma distributions, together with their convolutions, (suitable) mixtures, and symmetrizations, are typical examples of distributions satisfying Condition (iii), but normal and Cauchy distributions do not satisfy Condition (iii). Normal and Cauchy densities are examples of supersmooth densities, i.e., their characteristic functions decay exponentially fast as $|t| \rightarrow \infty$ (but mixtures of ordinary- and supersmooth densities are ordinary smooth). Furthermore, Theorem 1 in Hu and Ridder (2010) provides sufficient conditions in terms of $f_{U}$ under which the characteristic function of $U$ decays at most polynomially fast as $|t| \rightarrow \infty$. Specifically, if $f_{U}$ is range restricted of order $k$ in the sense that $f_{U}$ is supported on $[\underline{u}, \bar{u}]$ with either $\underline{u}$ or $\bar{u}$ finite, and has $k+2$ for $k \geq 0$ absolutely integrable derivatives $f_{U}^{(j)}$ with $\left|f_{U}^{(k)}(\underline{u})\right| \neq\left|f_{U}^{(k)}(\bar{u})\right|$ and $f_{U}^{(j-1)}(\underline{u})=f_{U}^{(j-1)}(\bar{u})$ for $j=1, \ldots, k$, then $\left|\varphi_{U}(t)\right|$ decays like $|t|^{-(k+1)}$ in the tail (Hu and Ridder, 2010, Theorem 1).

Condition (iv) is concerned with smoothness of the functions $f_{X}$ and $g$. Condition (v) is about a kernel function. Condition (vi) ensures that $\inf _{x \in I} f_{X}(x)>0$ (since $f_{X}$ is continuous) and 
$\inf _{x \in I} \mathrm{E}\left[\{Y-g(x)\}^{2} \mid W=x\right] f_{W}(x)>0$ (see the proof of Lemma A.4 (ii)). Note, that since $g f_{X}$ is bounded, we have that

$$
\|g\|_{I} \leq\left\|g f_{X}\right\|_{I} / \inf _{x \in I} f_{X}(x)<\infty .
$$

It is worth mentioning that under these conditions, we have that

$$
s_{n}^{2}(x)=\operatorname{Var}\left(\{Y-g(x)\} K_{n}\left((x-W) / h_{n}\right)\right) \sim h_{n}^{-2 \alpha+1}
$$

uniformly in $x \in I$ (see Lemma A.4, and the right hand side is larger by factor $h_{n}^{-2 \alpha}$ than the corresponding term in the error-free case (recall that, in standard kernel regression without measurement errors, the variance of $\epsilon K\left((x-X) / h_{n}\right)$ is $\left.\sim h_{n}\right)$. This results in slower rates of convergence of kernel regression estimators in presence of measurement errors than those in the error-free case, and the value of $\alpha$ is a key parameter that controls the difficulty of estimating $g$. Namely, the larger the value of $\alpha$ is, the more difficult estimation of $g$ will be. In other words, the value of $\alpha$ quantifies the degree of ill-posedness of estimation of $g$.

Condition (vii) restricts the bandwidth $h_{n}$ and the sample size $m$ from the measurement error distribution. The second condition in (12) restricts the growth rates of $m$, and is crucial for ensuring the negligibility of the effects of estimating the error characteristic function on the Gaussian approximation, which in turn establishes the bootstrap validity. This condition allows $m$ to be of smaller order than $n$, which in particular covers the repeated measurement setup discussed in Example 2.1. The last condition in (12) means that we are choosing undersmoothing bandwidths, that is, choosing bandwidths that are of smaller order than optimal rates for estimation of $g$ under the sup-norm loss. Inspection of the proof of Theorem 3.1 shows that without the last condition in (12), we have that

$$
\|\widehat{g}-g\|_{I}=O_{\mathrm{P}}\left\{h_{n}^{-\alpha}\left(n h_{n}\right)^{-1 / 2} \sqrt{\log \left(1 / h_{n}\right)}\right\}+O\left(h_{n}^{\beta}\right),
$$

where the $O\left(h_{n}^{\beta}\right)$ term comes from the deterministic bias. So, choosing $h_{n} \sim(n / \log n)^{-1 /(2 \alpha+2 \beta+1)}$ optimizes the rate on the right hand side, and the resulting rate of convergence of $\|\widehat{g}-g\|_{I}$ is $O_{\mathrm{P}}\left\{(n / \log n)^{-\beta /(2 \alpha+2 \beta+1)}\right\}$. The last condition in 12 requires to choose $h_{n}$ of smaller order than $(n / \log n)^{-1 /(2 \alpha+2 \beta+1)}$ (by $\log n$ factors), so that the "variance" term dominates the bias term. See Remark 3.3 for further discussions on the bias. For Condition (vii) to be non-void, we require $\beta>1 / 2$.

We first state a theorem that establishes that, under Assumption 3.1, the distribution of $\left\|\widehat{Z}_{n}\right\|_{I}=$ $\sup _{x \in I}\left|\widehat{Z}_{n}(x)\right|$, where $\left\{\widehat{Z}_{n}(x): x \in I\right\}$ is defined in $(4)$, can be approximated by that of the supremum of a certain Gaussian process, which is a building block for proving validity of the proposed confidence band. Recall that a Gaussian process $\{Z(x): x \in I\}$ indexed by $I$ is a tight random variable in $\ell^{\infty}(I)$ if and only if $I$ is totally bounded for the intrinsic $L^{2}$ pseudo-metric $\rho_{2}(x, y)=\sqrt{\mathrm{E}\left[\{Z(x)-Z(y)\}^{2}\right]}$ for $x, y \in I$, and $Z$ has sample paths almost surely uniformly $\rho_{2}$-continuous; see van der Vaart and Wellner (1996, p.41). Recall the process $Z_{n}^{*}$ defined in (3).

Theorem 3.1 (Gaussian approximation). Under Assumption 3.1, for each sufficiently large n, there exists a tight Gaussian process $Z_{n}^{G}$ in $\ell^{\infty}(I)$ with mean zero and the same covariance function 
as $\mathbf{Z}_{n}^{*}$, and such that as $n \rightarrow \infty$,

$$
\sup _{z \in \mathbb{R}}\left|\mathrm{P}\left\{\left\|\widehat{Z}_{n}\right\|_{I} \leq z\right\}-\mathrm{P}\left\{\left\|\mathrm{Z}_{n}^{G}\right\|_{I} \leq z\right\}\right| \rightarrow 0
$$

Theorem 3.1 derives an "intermediate" Gaussian approximation to the process $\widehat{Z}_{n}$, in the sense that the distribution of the approximating Gaussian process $Z_{n}^{G}$ depends on the sample size $n$. It could be possible to further show that, if $I$ is not a singleton, under additional conditions, for some sequences $a_{n}>0$ and $b_{n} \in \mathbb{R}, a_{n}\left(\left\|\widehat{Z}_{n}^{G}\right\|_{I}-b_{n}\right)$ converges in distribution to a Gumbel distribution. However, while it is mathematically intriguing, we avoid using the Gumbel approximation, since 1) the Gumbel approximation is slow and the coverage error of the resulting confidence band is of order $1 / \log n$ (see Hall, 1991), and 2) deriving the Gumbel approximation would require additional restrictive conditions on the measurement error distribution. For example, in a problem constructing confidence bands in deconvolution with known error distribution, Bissantz et al. (2007) derive a Gumbel approximation to the supremum deviation of the deconvolution kernel density estimator, thereby establishing a Smirnov-Bickel-Rosenblatt type theorem (Smirnov, 1950; Bickel and Rosenblatt, 1973) for the deconvolution kernel density estimator. But to do so, they require more restrictive conditions on the measurement error distribution than those in the present paper (see their Assumption 2).

The following theorem shows asymptotic validity of the proposed confidence band.

Theorem 3.2 (Validity of multiplier bootstrap confidence band). Under Assumption 3.1, as $n \rightarrow$ $\infty$

$$
\sup _{z \in \mathbb{R}}\left|\mathrm{P}\left\{\left\|\widehat{Z}_{n}^{\xi}\right\|_{I} \leq z \mid \mathcal{D}_{n}\right\}-\mathrm{P}\left\{\left\|\mathrm{Z}_{n}^{G}\right\|_{I} \leq z\right\}\right| \stackrel{\mathrm{P}}{\rightarrow} 0
$$

where $\mathbf{Z}_{n}^{G}$ is the Gaussian process in $\ell^{\infty}(I)$ given in Theorem 3.1. Therefore, for the confidence band $\widehat{\mathcal{C}}_{1-\tau}$ defined in (6), we have as $n \rightarrow \infty$,

$$
\mathrm{P}\left\{g(x) \in \widehat{\mathcal{C}}_{1-\tau}(x) \forall x \in I\right\}=1-\tau+o(1) .
$$

Finally, the supremum width of the band $\widehat{\mathcal{C}_{1-\tau}}$ is

$$
O_{\mathrm{P}}\left\{h_{n}^{-\alpha}\left(n h_{n}\right)^{-1 / 2} \sqrt{\log \left(1 / h_{n}\right)}\right\} .
$$

Remark 3.1. Inspection of the proof shows that the result (11) holds even when $\tau=\tau_{n} \downarrow 0$ as $n \rightarrow$ $\infty$. Furthermore, the supremum width of the band is $O_{\mathrm{P}}\left\{h_{n}^{-\alpha}\left(n h_{n}\right)^{-1 / 2} \sqrt{\log \left(1 / h_{n}\right) \vee \log \left(1 / \tau_{n}\right)}\right\}$.

Remark 3.2. For $v_{n} \sim(\log n)^{-1}$, take $h_{n}=v_{n}(n / \log n)^{-1 /(2 \alpha+2 \beta+1)}$; then the supremum width of the band $\widehat{\mathcal{C}}_{1-\tau}$ is $(n / \log n)^{-\beta /(2 \alpha+2 \beta+1)}(\log n)^{\alpha+1 / 2}$.

Remark 3.3 (Bias). For any nonparametric inference problem, how to deal with the deterministic bias is a delicate and difficult problem. See Section 5.7 in Wasserman (2006) for related discussions. In the present paper, we employ undersmoothing bandwidths so that the bias is negligible relative to the "variance" part. An alternative approach is to estimate the bias at each point, and construct a bias corrected confidence band. See, for example, Eubank and Speckman (1993) and Xia (1998) for the error-free case (more recent discussions regarding the problem of bias in nonparametric inference problems include Hall and Horowitz (2013), Chernozhukov et al. (2014b), Armstrong 
and Kolesár (2018), Calonico et al. (2018), and Schennach (2015); these papers do not cover EIV regression). However, in EIV regression, estimation of the bias is not quite attractive for a couple of reasons. First, the bias consists of higher order derivatives of $g$ and $f_{X}$, and estimation of these higher order derivatives is difficult, especially in the EIV case. This is because estimation of $g$ and $f_{X}$ is an ill-posed inverse problem and rates of convergence of the derivative estimators of $g$ and $f_{X}$ are even slower than those in the error-free case. As such, using a bias-corrected estimator would suffer from slower convergence rates and more complicated inference results. Second, one of popular kernels used in EIV regression and deconvolution is a flat top kernel (McMurry and Politis, 2004) which is an infinite order kernel, and if we use a flat top kernel, then the bias is not calculated in a closed form (e.g., Schennach (2004); Bissantz et al. (2007) use flat top kernels in their simulation studies). See Remark 1 in Bissantz et al. (2007) for a related issue in the deconvolution case.

Remark 3.4 (supersmooth case). In the present paper, we focus on the case where the measurement error density is ordinary smooth, similarly to Bissantz et al. (2007), Schmidt-Hieber et al. (2013), and Delaigle et al. (2015) that study inference in deconvolution and nonparametric EIV regression. If the measurement error density is supersmooth, i.e., its characteristic function decays exponentially fast as $|t| \rightarrow \infty$, then 1 ) in view of the pointwise asymptotic normality result in Fan and Masry (1992), the asymptotic behavior of the variance function $s_{n}^{2}(x)$ is much more complex. Indeed, as in Kato and Sasaki (2016), assume that the distribution of $U$ is supersmooth in the sense that $\varphi_{U}(t) \sim|t|^{\gamma_{0}} e^{-\nu|t|^{\gamma}}$ as $|t| \rightarrow \infty$ for some $\gamma>1, \gamma_{0} \in \mathbb{R}, \nu>0$, and the Fourier transform of the kernel function $\varphi_{K}$ is even, supported in $[-1,1]$, and $\varphi_{K}(1-t) \sim t^{\lambda}$ as $t \downarrow 0$ for some $\lambda \geq 0$; then it is expected that

$$
s_{n}^{2}(x) \sim h_{n}^{2 \gamma(1+\lambda)+2 \gamma_{0}} e^{2 \nu h_{n}^{-\gamma}} .
$$

See also van Es and Uh (2005). Importantly, the ratio of $1 / \inf _{|t| \leq h_{n}^{-1}}\left|\varphi_{U}(t)\right|$ over $\inf _{x \in I} \sigma_{n}(x)$ would be then larger in the supersmooth case than that in the ordinary smooth case; the ratio is $O\left(h_{n}^{-\gamma(1+\lambda)}\right)$ in the supersmooth case, while it is $O\left(h_{n}^{-1 / 2}\right)$ in the ordinary smooth case. This would require $m$ to be of larger order than $n$ (i.e., $m / n \rightarrow \infty$ ) to formally show validity of the confidence band in the supersmooth case. Analogous discussion can be found in Kato and Sasaki (2016) in the density deconvolution case. 2) Minimax rates of convergence for estimation of $g$ under the sup-norm loss are logarithmically slow (i.e., of the form $(\log n)^{-c}$ for some constant $c>0$ ), even when the measurement error distribution is assumed to be known (Fan and Truong, 1993). These difficulties prevent us from directly extending our analysis to the supersmooth case. Hence the supersmooth case is left for future research.

The proofs of Theorems 3.1 and 3.2 build on non-trivial applications of the intermediate Gaussian and multiplier bootstrap approximation theorems developed in Chernozhukov et al. (2014a b, 2016). However, we stress that Theorems 3.1 and 3.2 do not follow directly from the general theorems in Chernozhukov et al. (2014a b, 2016) and require substantial work. This is because 1) first of all, how to devise a multiplier bootstrap in EIV regression is not apparent, and as discussed in Remark 2.1 our construction of the multiplier process appears to be novel; 2) the "population" deconvolution kernel $K_{n}$ is implicitly defined via the Fourier inversion and substantially different 
from standard kernels in the error-free case; and 3) the deconvolution kernel $K_{n}$ is in fact unknown and estimated, so that its estimation error has to be taken into account.

An alternative standard technique to derive Gaussian approximations similar to (9) is to apply the Komlós-Major-Tusnády (KMT) strong approximation (Komlós et al., 1975). In a problem of constructing confidence bands in deconvolution with known error distribution, Bissantz et al. (2007) (and Schmidt-Hieber et al. (2013)) use the KMT approximation to derive Gaussian approximations to the deconvolution kernel density estimator. However, the KMT approximation is tailored to empirical processes indexed by univariate functions and hence is not applicable to our problem. Alternatively, we can use Rio's coupling (see Rio, 1994), but to apply Rio's coupling, we would have to assume (at least) that $Y$ is bounded (rather than finite fourth moment) and $K_{n}$ has total variation of order $h_{n}^{-\alpha}$ (which requires additional conditions on the measurement error distribution). By employing the techniques developed in Chernozhukov et al. (2014a b, 2016), we are able to avoid such restrictive conditions.

\section{Practical considerations}

4.1. Additional regularization. Estimation of the characteristic function, $\varphi_{U}$, is difficult in practice. Therefore, an additional regularization including a ridge parameter, for example, is useful for robustness. Following Delaigle et al. (2008, Sec. 4), we employ

$$
\widetilde{\varphi}_{U}(t)=\widehat{\varphi}_{U}(t) \cdot 1_{\{t \in \widehat{T}\}}+\left(1+\widehat{a}_{U} t^{2}\right)^{-\widehat{b}_{U}} \cdot 1_{\{t \notin \widehat{T}\}},
$$

where $\widehat{T}$ is the largest interval around 0 in which $\widehat{\varphi}_{U}$ is non-decreasing to the left of 0 and nonincreasing to the right of 0 , and $\left(a_{U}, b_{U}\right)=\left(\widehat{a}_{U}, \widehat{b}_{U}\right)$ fits the empirical second and fourth moments of $\eta$ with the characteristic function $t \mapsto\left(1+a_{U} t^{2}\right)^{-b_{U}}$, i.e., $\left(\widehat{a}_{U}, \widehat{b}_{U}\right)$ are chosen to satisfy $2 \widehat{a}_{U} \widehat{b}_{U}=$ $\frac{1}{m} \sum_{j=1}^{m} \eta_{j}^{2}$ and $12 \widehat{a}_{U}^{2} \widehat{b}_{U}\left(\widehat{b}_{U}+1\right)=\frac{1}{m} \sum_{j=1}^{m} \eta_{j}^{4}$. In case where these coefficients are negative, we set $\widehat{b}_{U}=1$ following Delaigle et al. (2008, Sec. 4). That is, we set

$$
\begin{aligned}
& \widehat{a}_{U}=\frac{1}{2 \widehat{b}_{U}} \cdot \frac{1}{m} \sum_{j=1}^{m} \eta_{j}^{2} \text { and } \\
& \widehat{b}_{U}= \begin{cases}1 & \text { if } \frac{1}{m} \sum_{j=1}^{m} \eta_{j}^{4} \leq 3\left(\frac{1}{m} \sum_{j=1}^{m} \eta_{j}^{2}\right)^{2} \\
\frac{3\left(\frac{1}{m} \sum_{j=1}^{m} \eta_{j}^{2}\right)^{2}}{\frac{1}{m} \sum_{j=1}^{m} \eta_{j}^{4}-3\left(\frac{1}{m} \sum_{j=1}^{m} \eta_{j}^{2}\right)^{2}} & \text { if } \frac{1}{m} \sum_{j=1}^{m} \eta_{j}^{4}>3\left(\frac{1}{m} \sum_{j=1}^{m} \eta_{j}^{2}\right)^{2}\end{cases}
\end{aligned}
$$

With this approach, the robustness is achieved by a fully data-driven manner. In simulation studies and real data analysis below, we use the estimated deconvolution kernel with this additional regularization:

$$
\widetilde{K}_{n}(x)=\frac{1}{2 \pi} \int_{\mathbb{R}} e^{-i t x} \frac{\varphi_{K}(t)}{\widetilde{\varphi}_{U}(t / h)} d t .
$$

4.2. Bandwidth Selection. For another practical consideration, we present a bandwidth selection rule based on the SIMEX methods (Delaigle and Hall, 2008; Delaigle et al., 2015). The selection

procedure consists of two steps. In the first step, we choose an optimal bandwidth $\widetilde{h}_{n}$ in terms of balancing the supremum squared bias and the supremum variance (Section 4.2.1). In the second 
step, we choose a scaling factor $\widetilde{\chi}_{n}$ such that $\widetilde{\chi}_{n} \widetilde{h}_{n}$ optimizes the coverage probability with respect to the nominal one (Section 4.2.2).

4.2.1. Optimal bandwidth selection. To clarify the dependence on a candidate bandwidth $h$, write $\left.K(x ; h)=\frac{1}{2 \pi} \int_{\mathbb{R}} e^{-i t x} \frac{\varphi_{K}(t)}{\varphi_{U}(t / h)} d t, s^{2}(x ; h)=\operatorname{Var}(\{Y-g(x)\} K(x-W) / h ; h)\right)$, and $A(x ; h)=\mathrm{E}[\{Y-$ $g(x)\} K((x-W) / h ; h)]$. An optimal choice $h_{n}$ (with the $\log n$ factor ignored) minimizes the criterion function $\Gamma_{n}$ defined by $\Gamma_{n}(h)=\left\|A^{2}(\cdot ; h)\right\|_{I}+\left\|s^{2}(\cdot ; h) / n\right\|_{I}$. If the true function $g$ were known, then a natural estimator of the optimal bandwidth would be $\widetilde{h}_{n}=\arg \min _{h>0}\left\|\widetilde{A}_{n}^{2}(\cdot ; h)\right\|_{I}+\left\|\widetilde{s}_{n}^{2}(\cdot ; h) / n\right\|_{I}$, where

$$
\begin{aligned}
& \widetilde{s}_{n}^{2}(x ; h)=\frac{1}{n} \sum_{j=1}^{n}\left\{Y_{j}-g(x)\right\}^{2} \widetilde{K}_{n}^{2}\left(\left(x-W_{j}\right) / h ; h\right)-\widetilde{A}_{n}^{2}(x ; h) \text { and } \\
& \widetilde{A}_{n}(x ; h)=\frac{1}{n} \sum_{j=1}^{n}\left\{Y_{j}-g(x)\right\} \widetilde{K}_{n}\left(\left(x-W_{j}\right) / h ; h\right) .
\end{aligned}
$$

However, this is an infeasible procedure because we do not know the true function $g$ nor the distribution of $(Y, X)$ in practice. Therefore, adapting Delaigle and Hall (2008) to our framework, we use the SIMEX method outlined below to estimate the optimal $h_{n}$.

Let $\left\{U_{1}^{\dagger}, \ldots, U_{n}^{\dagger}\right\}$ and $\left\{U_{1}^{\dagger \dagger}, \ldots, U_{n}^{\dagger \dagger}\right\}$ be independent samples drawn with replacement from $\left\{\eta_{1}, \ldots, \eta_{m}\right\}$, independently of the data $\left\{\left(Y_{1}, W_{1}\right), \ldots,\left(Y_{n}, W_{n}\right)\right\}$. Write $W_{j}^{\dagger}=W_{j}+U_{j}^{\dagger}$ and $W_{j}^{\dagger \dagger}=W_{j}+U_{j}^{\dagger}+U_{j}^{\dagger \dagger}$ for each $j \in\{1, \ldots, n\}$. As counterparts of $s^{2}(x ; h)$ and $A(x ; h)$, consider $s^{\dagger 2}(x ; h)=\operatorname{Var}\left(\left\{Y-g_{1}(x)\right\} K\left(\left(x-W^{\dagger}\right) / h ; h\right)\right), A^{\dagger}(x ; h)=\mathrm{E}\left[\left\{Y-g_{1}(x)\right\} K\left(\left(x-W^{\dagger}\right) / h ; h\right)\right]$, $s^{\dagger \dagger 2}(x ; h)=\operatorname{Var}\left(\left\{Y-g_{2}(x)\right\} K\left(\left(x-W^{\dagger \dagger}\right) / h ; h\right)\right)$, and $A^{\dagger \dagger}(x ; h)=\mathrm{E}\left[\left\{Y-g_{2}(x)\right\} K\left(\left(x-W^{\dagger \dagger}\right) / h ; h\right)\right]$, where $g_{1}(x)=\mathrm{E}[Y \mid W=x]$ and $g_{2}(x)=\mathrm{E}\left[Y \mid W^{\dagger}=x\right]$. Substituting estimates and the additional regularization suggested at the end of Section 4.1, we can compute

$$
\begin{aligned}
& \widetilde{s}_{n}^{\dagger 2}(x ; h)=\frac{1}{n} \sum_{j=1}^{n}\left\{Y_{j}-\widetilde{g}_{1}(x)\right\}^{2} \widetilde{K}_{n}^{2}\left(\left(x-W_{j}^{\dagger}\right) / h ; h\right)-\widetilde{A}_{n}^{\dagger 2}(x ; h) \text { where } \\
& \widetilde{A}_{n}^{\dagger}(x ; h)=\frac{1}{n} \sum_{j=1}^{n}\left\{Y_{j}-\widetilde{g}_{1}(x)\right\} \widetilde{K}_{n}\left(\left(x-W_{j}^{\dagger}\right) / h ; h\right), \text { and } \\
& \widetilde{s}_{n}^{\dagger \dagger}(x ; h)=\frac{1}{n} \sum_{j=1}^{n}\left\{Y_{j}-\widetilde{g}_{2}(x)\right\}^{2} \widetilde{K}_{n}^{2}\left(\left(x-W_{j}^{\dagger \dagger}\right) / h ; h\right)-\widetilde{A}_{n}^{\dagger \dagger 2}(x ; h) \text { where } \\
& \widetilde{A}_{n}^{\dagger \dagger}(x ; h)=\frac{1}{n} \sum_{j=1}^{n}\left\{Y_{j}-\widetilde{g}_{2}(x)\right\} \widetilde{K}_{n}\left(\left(x-W_{j}^{\dagger \dagger}\right) / h ; h\right),
\end{aligned}
$$

with $\widetilde{g}_{1}$ and $\widetilde{g}_{2}$ denoting error-free estimates of $g_{1}$ and $g_{2}$, respectively, which are feasible with the observed and simulated data $\left\{\left(Y_{1}, W_{1}, W_{1}^{\dagger}\right), \ldots,\left(Y_{n}, W_{n}, W_{n}^{\dagger}\right)\right\}$. Let

$$
\begin{aligned}
\widetilde{\Gamma}_{n}^{\dagger}(h) & =\left\|\widetilde{A}_{n}^{\dagger 2}(\cdot ; h)\right\|_{I}+\left\|\widetilde{s}_{n}^{\dagger 2}(\cdot ; h) / n\right\|_{I} \text { and } \\
\widetilde{\Gamma}_{n}^{\dagger \dagger}(h) & =\left\|\widetilde{A}_{n}^{\dagger \dagger 2}(\cdot ; h)\right\|_{I}+\left\|\widetilde{s}_{n}^{\dagger \dagger 2}(\cdot ; h) / n\right\|_{I} .
\end{aligned}
$$

Since $\widetilde{\Gamma}_{n}^{\dagger}(h)$ and $\widetilde{\Gamma}_{n}^{\dagger \dagger}(h)$ are affected by the simulated data, $\left\{U_{1}^{\dagger}, \ldots, U_{n}^{\dagger}\right\}$ and $\left\{U_{1}^{\dagger \dagger}, \ldots, U_{n}^{\dagger \dagger}\right\}$, we average over a large number $B$ of their versions, $\left\{\widetilde{\Gamma}_{n, b}^{\dagger}(h)\right\}_{b=1}^{B}$ and $\left\{\widetilde{\Gamma}_{n, b}^{\dagger \dagger}(h)\right\}_{b=1}^{B}$. We then estimate 
the optimal bandwidths

$$
\widetilde{h}_{n, 1}=\arg \min _{h>0} \frac{1}{B} \sum_{b=1}^{B} \widetilde{\Gamma}_{n, b}^{\dagger}(h) \quad \text { and } \quad \widetilde{h}_{n, 2}=\arg \min _{h>0} \frac{1}{B} \sum_{b=1}^{B} \widetilde{\Gamma}_{n, b}^{\dagger \dagger}(h)
$$

for $g_{1}$ and $g_{2}$, respectively. Note that " $W^{\ddagger}$ measures $W^{\dagger}$ in the same way that $W^{\dagger}$ measures $W$ and $W$ measures $X "$ (Delaigle and Hall, 2008, Sec. 2.3). We therefore expect that $\widetilde{h}_{n, 2} / \widetilde{h}_{n, 1}$ is similar to $\widetilde{h}_{n, 1} / \widetilde{h}_{n}$. This motivates the selection of $h_{n}$ by the linear backward extrapolation

$$
\widetilde{h}_{n}=\widetilde{h}_{n, 1}^{2} / \widetilde{h}_{n, 2}
$$

4.2.2. Coverage probability optimal bandwidth selection. The bandwidth chosen in Section 4.2.1 is based on balancing the bias and variance, and therefore fails to provide an asymptotically valid inference result. In this section, we discuss a fully data-driven procedure of choosing a coverageprobability optimal choice of bandwidth. To clarify the dependence on a candidate bandwidth $h$, write the confidence band by $\widehat{\mathcal{C}}_{1-\tau}(x ; h), x \in I$. We define the ideal bandwidth to be $\chi_{n} h_{n}$ that minimizes the coverage error as $\chi_{n}=\arg \min _{\chi \geq 1}\left|\mathrm{P}\left(g(x) \in \widehat{\mathcal{C}}_{1-\tau}\left(x ; \chi h_{n}\right) \forall x \in I\right)-(1-\tau)\right|$. In practice, we do not know $g$ nor the distribution of $(Y, X)$. We therefore adapt the SIMEX method of Delaigle et al. (2015, Sec. 3.2) to our framework.

The same notations for the simulated samples from Section 4.2.1 carry over to the current section. Let $\widehat{\mathcal{C}}_{1-\tau}^{\dagger}(x ; h)$ and $\widehat{\mathcal{C}}_{1-\tau}^{\dagger \dagger}(x ; h), x \in I$ denote the confidence bands for $g_{1}$ and $g_{2}$, respectively, computed using the samples $\left\{\left(Y_{1}, W_{1}^{\dagger}\right), \ldots,\left(Y_{n}, W_{n}^{\dagger}\right)\right\}$ and $\left\{\left(Y_{1}, W_{1}^{\dagger \dagger}\right), \ldots,\left(Y_{n}, W_{n}^{\dagger \dagger}\right)\right\}$, respectively. We repeat the simulation for large number $B$ of times to construct versions, $\left\{\widehat{\mathcal{C}}_{1-\tau, b}^{\dagger}(x ; h)\right\}_{b=1}^{B}$ and $\left\{\widehat{\mathcal{C}}_{1-\tau, b}^{\dagger \dagger}(x ; h)\right\}_{b=1}^{B}$, and estimate the coverage probabilities for $g_{1}$ and $g_{2}$ by

$$
\begin{aligned}
& \widehat{C P}_{1-\tau}^{\dagger}(h)=\frac{1}{B} \sum_{b=1}^{B} 1_{\left\{\widetilde{g}_{1}(x) \in \widehat{\mathcal{C}}_{1-\tau, b}^{\dagger}(x ; h) \forall x \in I\right\}} \quad \text { and } \\
& \widehat{C P}_{1-\tau}^{\dagger \dagger}(h)=\frac{1}{B} \sum_{b=1}^{B} 1_{\left\{\widetilde{g}_{2, b}(x) \in \widehat{\mathcal{C}}_{1-\tau, b}^{\dagger \dagger}(x ; h) \forall x \in I\right\}},
\end{aligned}
$$

where $\widetilde{g}_{1}$ and $\widetilde{g}_{2, b}$ denote error-free estimates of $g_{1}$ and $g_{2}$, respectively, which are feasible with the observed data $\left\{\left(Y_{1}, W_{1}\right), \ldots,\left(Y_{n}, W_{n}\right)\right\}$ and $b$-th simulated data $\left\{\left(Y_{1}, W_{1}^{\dagger}\right), \ldots,\left(Y_{n}, W_{n}^{\dagger}\right)\right\}$, respectively.

We then estimate the coverage-error-optimal bandwidths for $g_{1}$ and $g_{2}$ by $\widetilde{\chi}_{n, 1} \widetilde{h}_{n, 1}$ and $\widetilde{\chi}_{n, 2} \widetilde{h}_{n, 2}$, respectively, where

$$
\begin{aligned}
& \widetilde{\chi}_{n, 1}=\arg \min _{\chi \geq 1}\left|\widehat{C P}_{1-\tau}^{\dagger}\left(\chi \widetilde{h}_{n, 1}\right)-(1-\tau)\right| \quad \text { and } \\
& \widetilde{\chi}_{n, 2}=\arg \min _{\chi \geq 1}\left|\widehat{C P}_{1-\tau}^{\dagger \dagger}\left(\chi \widetilde{h}_{n, 2}\right)-(1-\tau)\right| .
\end{aligned}
$$

Finally, the coverage-error-optimal bandwidth for $g$ may be estimated by $\widetilde{\chi}_{n} \widetilde{h}_{n}$ where, following a similar reasoning to the one made at the end of Section 4.2.1, $\widetilde{\chi}_{n}$ is given by the linear backward extrapolation $\tilde{\chi}_{n}=\widetilde{\chi}_{n, 1}^{2} / \widetilde{\chi}_{n, 2}$. 
Remark 4.1. The bandwidth selection procedure presented above consists of two steps: the first step chooses the optimal bandwidth $\widetilde{h}_{n}$ due to Delaigle and Hall (2008), and the second step chooses the scaling factor $\widetilde{\chi}_{n}$ to optimize the coverage probability due to Delaigle et al. (2015). To our best knowledge, this data-driven selection procedure has not been formally proven to be consistent with theoretical requirements of inference methods even under the simpler settings covered in the existing literature. In order to ensure that the theoretical results of the paper still hold, we require the existence of a deterministic bandwidth $h_{n}$ such that $\widetilde{\chi}_{n} \widetilde{h}_{n} / h_{n}=1+o_{p}\left(h_{n}^{-1 / 2}\right)$. Indeed, the two-step SIMEX procedure produces a complicated stochastic sequence, and we hence remark that a theoretical investigation of this practical issue by itself deserves a future research.

\section{Simulation Studies}

5.1. Simulation Framework. We consider two data generating models, reflecting two common patterns of data availability. For the first model, the observed data $\mathcal{D}_{n}=\left\{\left(Y_{j}, W_{j}, \eta_{j}\right)\right\}_{j=1}^{n}$ is constructed by

$$
\text { Model } 1: Y_{j}=g\left(X_{j}\right)+\epsilon_{j}, W_{j}=X_{j}+U_{j}, \eta_{j} \stackrel{d}{=} U_{j}, j=1, \ldots, n
$$

where $X_{j}, \epsilon_{j}, U_{j}$, and $\eta_{j}$ are independent with $X_{j} \sim N\left(0, \sigma_{X}^{2}\right), \epsilon_{j} \sim N(0,1)$ and $U_{j} \stackrel{d}{=} \eta_{j} \sim$ Laplace $\left(0,2^{-1 / 2}\right)$. The characteristic function of $U_{j}$ is $\varphi_{U}(t)=\left(1+t^{2} / 2\right)^{-1}$, which is non-vanishing on $\mathbb{R}$ and ordinary smooth of order $\alpha=2$. The signal-to-noise ratio is $\sqrt{\operatorname{Var}(X) / \operatorname{Var}(U)}=\sigma_{X}$.

The second model considers the repeated measurement setup:

$$
\text { Model } 2: Y_{j}=g\left(X_{j}\right)+\epsilon_{j}, W_{j}^{(k)}=X_{j}+U_{j}^{(k)}, j=1, \ldots, n ; k=1,2,
$$

where $X_{j}, \epsilon_{j}, U_{j}^{(1)}$, and $U_{j}^{(2)}$ are independent with $X_{j} \sim N\left(0, \sigma_{X}^{2}\right), \epsilon_{j} \sim N(0,1)$ and $U_{j}^{(k)} \sim$ Laplace $(0,1)$. We observe $\left\{\left(Y_{j}, W_{j}^{(1)}, W_{j}^{(2)}\right)\right\}_{j=1}^{n}$. By defining $W_{j}:=\left(W_{j}^{(1)}+W_{j}^{(2)}\right) / 2$ and $\eta_{j}:=$ $\left(W_{j}^{(1)}-W_{j}^{(2)}\right) / 2$, we obtain the generated data $\mathcal{D}_{n}=\left\{\left(Y_{j}, W_{j}, \eta_{j}\right)\right\}_{j=1}^{n}$ such that $W_{j}=X_{j}+U_{j}$ with $U_{j}=\left(U_{1}+U_{2}\right) / 2 \stackrel{d}{=} \eta_{j}$. For Model 2, the characteristic function of $U_{j}$ is $\varphi_{U}(t)=\left(1+t^{2} / 4\right)^{-2}$, which is non-vanishing on $\mathbb{R}$ and ordinary smooth with order $\alpha=4$. The signal-to-noise ratio is $\sqrt{\operatorname{Var}(X) / \operatorname{Var}(U)}=\sigma_{X}$.

Simulations are run across five different specifications of $g$ and two alternative values of $\sigma_{X}$. The five specifications of $g$ are $g(x)=x, g(x)=x^{2}, g(x)=x^{3}, g(x)=\sin (x)$, and $g(x)=\cos (x)$. The two alternative values of $\sigma_{X}$ are $\{1 / \sqrt{1 / 4}, 1 / \sqrt{1 / 3}\}$ for both Model 1 and Model 2. The variances of the errors are one fourth (25\%) and one third (33\%) that of $X$ with these values of $\sigma_{X}$.

We use Monte Carlo simulations to evaluate the coverage probabilities of our confidence bands for $g$ on the interval $I=\left[-\sigma_{X}, \sigma_{X}\right]$. To assess how informative these confidence bands are, we also record and present the median statistics of the average band lengths on $I$, and also visually illustrate realized bands via figures. We use the kernel function $K$ defined by its Fourier transform $\varphi_{K}$ given by

$$
\varphi_{K}(t)=1_{\{|t| \leq c\}}+\exp \left\{\frac{-b \exp \left(-b /(|t|-c)^{2}\right)}{(|t|-1)^{2}}\right\} 1_{\{c<|t|<1\}},
$$

where $b=1$ and $c=0.05$ (cf. McMurry and Politis, 2004; Bissantz et al., 2007). The function $\varphi_{K}$ is infinitely differentiable with support $[-1,1], \varphi^{(\ell)}(0)=0$ for any $\ell \geq 1$, and its inverse 
Fourier transform $K$ is real-valued and integrable with $\int_{\mathbb{R}} K(x) d x=1$. We follow the additional regularization discussed in Section 4.1 and the bandwidth selection rule discussed in Section 4.2 .

5.2. Simulation Results. Since the simulation results are qualitatively similar for all specifications of the function $g$, we only discuss simulation results for $g(x)=x^{3}$ and $g(x)=\sin (x)$ in the present section. Results for the remaining functions, $g(x)=x, g(x)=x^{2}$, and $g(x)=\cos (x)$, can be found in Appendix B. Table 1 presents simulated uniform coverage probabilities for (A) $g(x)=x^{3}$ and (B) $g(x)=\sin (x)$ by estimated confidence bands in $I=\left[-\sigma_{X}, \sigma_{X}\right]$, and the median statistics of the average band lengths on $I$ based on 1,000 Monte Carlo iterations. Observe that the simulated coverage probabilities approaches the nominal probabilities as the sample size increases, and they are reasonably close for the sample size of $n=400$. Also observe that the median statistics of the average band length tend to decrease as the sample size increases.

Note that our method applies even when $I$ is a singleton. In this case, a uniform confidence band boils down to a pointwise one. We next run simulations for the pointwise cinfidence band with $I=\{x\}$ for each location $x \in\left[-\sigma_{X}, \sigma_{X}\right]$. The second column group of Table 2 summarizes simulation results regarding the pointwise band. For comparisons, the first column group of Table 2 also displays the simulation results regarding the uniform band copied from Table 1. For the pointwise band, in addition to the uniform coverage probabilities and the medians of the average band lengths, we also report the average fraction of the length of the subset of $I$ on which the pointwise band covers the true function $g$. Notice that the uniform coverage probabilities for the pointwise band fall short of the nominal probabilities to large extents, and they deviate further as the sample size increases. These results motivate the attractiveness of the uniform band for the purpose of assessing the global shape of the true function $g$. With this said, we also observe that average covered portions by the poitwise band are fairly close to the nominal probabilities, which is consistent with a popular (but unclear) belief in the literature. Theoretical discussions of the latter observation is out of the scope of this paper, but we would like to make this remark to suggest a directin of future research.

The informativeness of the uniform confidence bands are better assessed with visual presentations, besides the band lengths reported in the table. Figure 1 displays realizations of estimates confidence bands for functions, $g(x)=x^{3}$ and $g(x)=\sin (x)$, on $I$ for each of the two error variance ratios (1/4 and 1/3) under Model 1 (results for the remaining functions and/or model can be found in Appendix B]. Observe that the realized confidence bands are precise enough to provide us with ideas about the possible shapes of the true regression functions. We may distinguish the five functions by the realized shapes of the confidence bands. For these particular realizations, it is not possible to conclude that the band length shrink as the sample size increases, but the band lengths reported in Table 1 complement the figure to this end.

\section{Real data analysis}

In this section, we apply our method to real data, and draw confidence bands for nonparametric regressions of medical prescription expenditure on body mass index (BMI). We combine the following two data sets. The National Health and Nutrition Examination Survey (NHANES) provides 


\begin{tabular}{|c|c|c|c|c|c|c|}
\hline \multicolumn{3}{|c|}{ (A) Regression: $g(x)=x^{3}$} & \multicolumn{2}{|c|}{$\begin{array}{l}\text { Error Variance } \\
=1 / 4(25 \%)\end{array}$} & \multicolumn{2}{|c|}{$\begin{array}{l}\text { Error Variance } \\
=1 / 3(33 \%)\end{array}$} \\
\hline Model & Probability & Size $(n)$ & Coverage & Length & Coverage & Length \\
\hline \multirow[t]{3}{*}{1} & 0.900 & 100 & 0.886 & 14.802 & 0.894 & 10.294 \\
\hline & & 200 & 0.931 & 10.211 & 0.917 & 7.222 \\
\hline & & 400 & 0.914 & 7.391 & 0.912 & 5.286 \\
\hline \multirow[t]{3}{*}{1} & 0.950 & 100 & 0.903 & 16.527 & 0.919 & 11.449 \\
\hline & & 200 & 0.963 & 11.405 & 0.947 & 8.029 \\
\hline & & 400 & 0.955 & 8.215 & 0.950 & 5.883 \\
\hline \multirow[t]{3}{*}{2} & 0.900 & 100 & 0.930 & 16.587 & 0.927 & 11.554 \\
\hline & & 200 & 0.949 & 11.030 & 0.935 & 7.763 \\
\hline & & 400 & 0.925 & 7.288 & 0.893 & 5.097 \\
\hline \multirow[t]{3}{*}{2} & 0.950 & 100 & 0.951 & 18.428 & 0.950 & 12.870 \\
\hline & & 200 & 0.974 & 12.271 & 0.962 & 8.684 \\
\hline & & 400 & 0.956 & 8.149 & 0.941 & 5.691 \\
\hline
\end{tabular}

\begin{tabular}{|c|c|c|c|c|c|c|}
\hline \multicolumn{3}{|c|}{ (B) Regression: $g(x)=\sin (x)$} & \multirow{2}{*}{\multicolumn{2}{|c|}{$\begin{array}{l}\text { Error Variance } \\
=1 / 4(25 \%)\end{array}$}} & \multirow{2}{*}{\multicolumn{2}{|c|}{$\begin{array}{l}\text { Error Variance } \\
=1 / 3(33 \%)\end{array}$}} \\
\hline \multirow[b]{2}{*}{ Model } & \multirow{2}{*}{$\begin{array}{c}\text { Nominal } \\
\text { Probability }\end{array}$} & \multirow{2}{*}{$\begin{array}{l}\text { Sample } \\
\text { Size }(n)\end{array}$} & & & & \\
\hline & & & Coverage & Length & Coverage & Length \\
\hline \multirow[t]{3}{*}{1} & 0.900 & 100 & 0.862 & 2.134 & 0.866 & 2.363 \\
\hline & & 200 & 0.890 & 1.619 & 0.874 & 1.512 \\
\hline & & 400 & 0.886 & 1.194 & 0.889 & 1.142 \\
\hline \multirow[t]{3}{*}{1} & 0.950 & 100 & 0.904 & 2.369 & 0.906 & 2.621 \\
\hline & & 200 & 0.932 & 1.796 & 0.915 & 1.682 \\
\hline & & 400 & 0.940 & 1.321 & 0.934 & 1.271 \\
\hline \multirow[t]{3}{*}{2} & 0.900 & 100 & 0.849 & 2.049 & 0.863 & 2.321 \\
\hline & & 200 & 0.883 & 1.636 & 0.874 & 1.526 \\
\hline & & 400 & 0.897 & 1.139 & 0.871 & 1.068 \\
\hline \multirow[t]{3}{*}{2} & 0.950 & 100 & 0.900 & 2.273 & 0.901 & 2.572 \\
\hline & & 200 & 0.931 & 1.820 & 0.923 & 1.697 \\
\hline & & 400 & 0.944 & 1.267 & 0.918 & 1.190 \\
\hline
\end{tabular}

TABLE 1. Simulated uniform coverage probabilities of (A) $g(x)=x^{3}$ and (B) $g(x)=\sin (x)$ by estimated confidence bands in $I=\left[-\sigma_{X}, \sigma_{X}\right]$ under normally distributed $X$ and Laplace distributed $U$. Also reported are the medians of the average band lengths on $I$. The simulated probabilities and lengths are computed for each of the two nominal coverage probabilities, $90 \%$ and $95 \%$, based on 1,000 Monte Carlo iterations. 


\begin{tabular}{|c|c|c|c|c|c|c|c|}
\hline \multicolumn{3}{|c|}{ (A) Regression: $g(x)=x^{3}$} & \multicolumn{2}{|c|}{ Uniform Band } & \multicolumn{3}{|c|}{ Pointwise Band } \\
\hline Error & Nominal & Sample & Uniform & Band & Uniform & Covered & Band \\
\hline Variance & Probability & Size $(n)$ & Coverage & Length & Coverage & Portion & Length \\
\hline $1 / 4$ & 0.900 & 100 & 0.886 & 14.802 & 0.618 & 0.935 & 9.727 \\
\hline$(25 \%)$ & & 200 & 0.931 & 10.211 & 0.593 & 0.923 & 6.968 \\
\hline & & 400 & 0.914 & 7.391 & 0.555 & 0.904 & 4.953 \\
\hline $1 / 4$ & 0.950 & 100 & 0.903 & 16.527 & 0.794 & 0.973 & 11.583 \\
\hline$(25 \%)$ & & 200 & 0.963 & 11.405 & 0.780 & 0.966 & 8.295 \\
\hline & & 400 & 0.955 & 8.215 & 0.733 & 0.954 & 5.894 \\
\hline $1 / 3$ & 0.900 & 100 & 0.894 & 10.294 & 0.628 & 0.933 & 6.797 \\
\hline$(33 \%)$ & & 200 & 0.917 & 7.222 & 0.577 & 0.914 & 5.012 \\
\hline & & 400 & 0.912 & 5.286 & 0.542 & 0.899 & 3.577 \\
\hline $1 / 3$ & 0.950 & 100 & 0.919 & 11.449 & 0.802 & 0.970 & 8.131 \\
\hline$(33 \%)$ & & 200 & 0.947 & 8.029 & 0.768 & 0.962 & 5.989 \\
\hline & & 400 & 0.950 & 5.883 & 0.728 & 0.951 & 4.259 \\
\hline
\end{tabular}

\begin{tabular}{|c|c|c|c|c|c|c|c|}
\hline \multicolumn{3}{|c|}{ (B) Regression: $g(x)=\sin (x)$} & \multicolumn{2}{|c|}{ Uniform Band } & \multicolumn{3}{|c|}{ Pointwise Band } \\
\hline Error & Nominal & Sample & Uniform & Band & Uniform & Covered & Band \\
\hline Variance & Probability & Size $(n)$ & Coverage & Length & Coverage & Portion & Length \\
\hline \multirow{3}{*}{$\begin{array}{c}1 / 4 \\
(25 \%)\end{array}$} & 0.900 & 100 & 0.862 & 2.134 & 0.544 & 0.908 & 1.421 \\
\hline & & 200 & 0.890 & 1.619 & 0.538 & 0.912 & 1.053 \\
\hline & & 400 & 0.886 & 1.194 & 0.536 & 0.908 & 0.790 \\
\hline \multirow{3}{*}{$\begin{array}{c}1 / 4 \\
(25 \%)\end{array}$} & 0.950 & 100 & 0.904 & 2.369 & 0.700 & 0.948 & 1.691 \\
\hline & & 200 & 0.932 & 1.796 & 0.725 & 0.955 & 1.254 \\
\hline & & 400 & 0.940 & 1.321 & 0.716 & 0.953 & 0.939 \\
\hline \multirow{3}{*}{$\begin{array}{c}1 / 3 \\
(33 \%)\end{array}$} & 0.900 & 100 & 0.866 & 2.363 & 0.541 & 0.901 & 1.392 \\
\hline & & 200 & 0.874 & 1.512 & 0.550 & 0.903 & 1.050 \\
\hline & & 400 & 0.889 & 1.142 & 0.521 & 0.897 & 0.750 \\
\hline \multirow{3}{*}{$\begin{array}{c}1 / 3 \\
(33 \%)\end{array}$} & 0.950 & 100 & 0.906 & 2.621 & 0.692 & 0.943 & 1.654 \\
\hline & & 200 & 0.915 & 1.682 & 0.706 & 0.948 & 1.252 \\
\hline & & 400 & 0.934 & 1.271 & 0.714 & 0.947 & 0.893 \\
\hline
\end{tabular}

TABLE 2. Simulated uniform coverage probabilities of (A) $g(x)=x^{3}$ and (B) $g(x)=$ $\sin (x)$ by estimated uniform and pointwise confidence bands in $\left[-\sigma_{X}, \sigma_{X}\right]$ under normally distributed $X$ and Laplace distributed $U$. The middle column under the column group of pointwise band displays the fraction of the length of the subset of $I$ on which the pointwise band contains the true function $g$. Also reported are the medians of the average band lengths on $I$. The simulated probabilities and lengths are computed for each of the two nominal coverage probabilities, $90 \%$ and $95 \%$, based on 1,000 Monte Carlo iterations. 
$n=200$

$g(x)=x^{3}$

$\mathrm{EV}=1 / 4(25 \%)$

$g(x)=x^{3}$

$\mathrm{EV}=1 / 3(33 \%)$
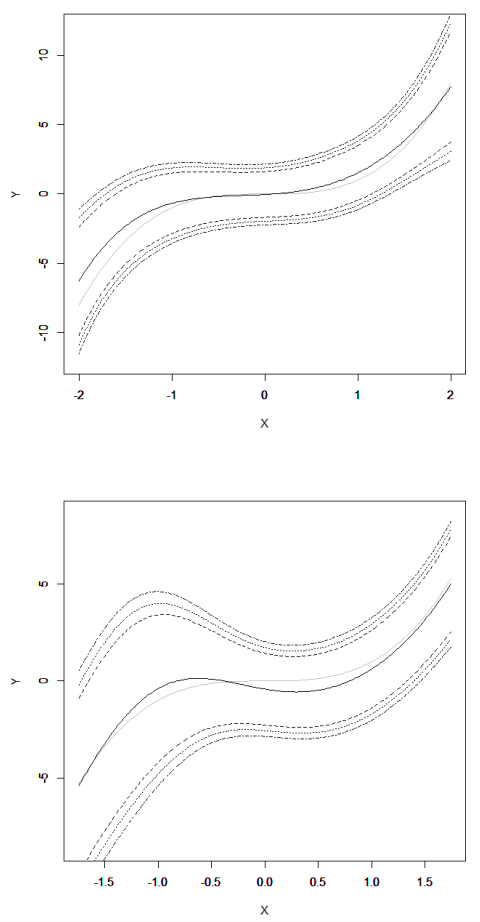

$g(x)=\sin (x)$

$\mathrm{EV}=1 / 4(25 \%)$
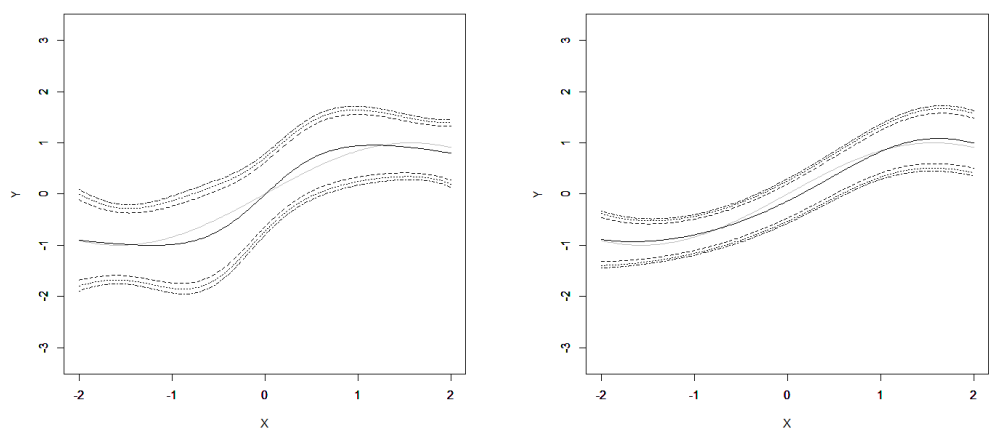

$g(x)=\sin (x)$

$\mathrm{EV}=1 / 3(33 \%)$

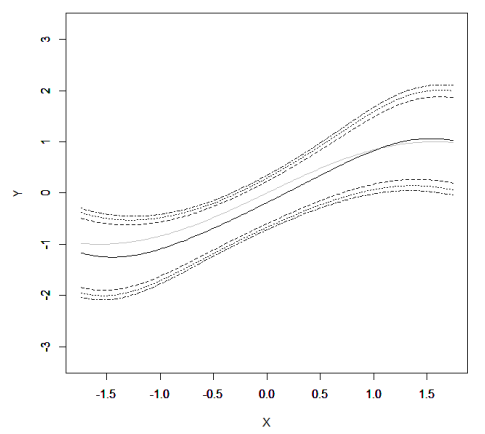

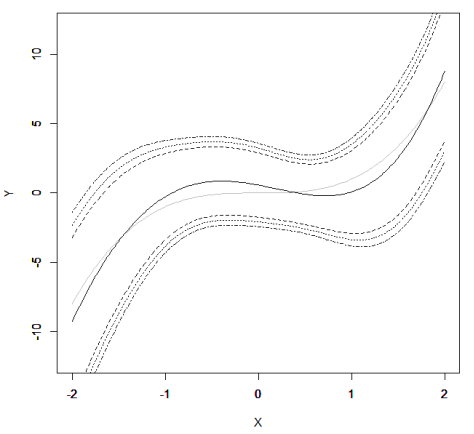

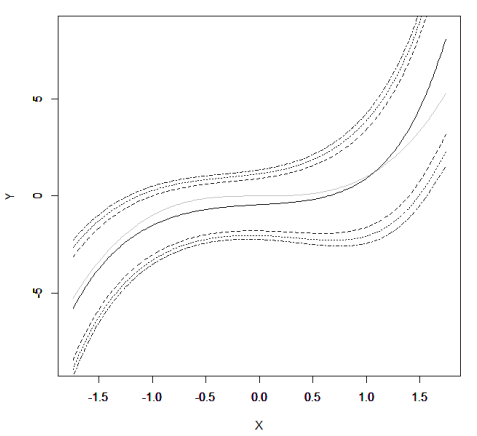

$n=400$

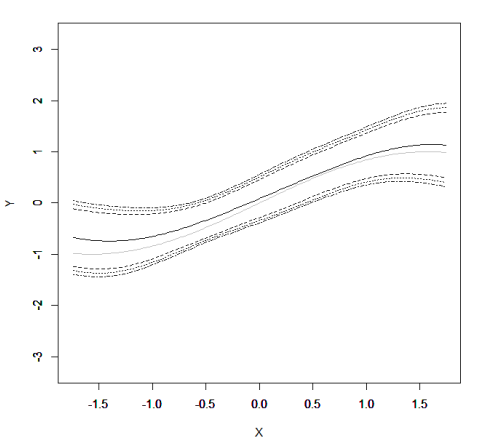

Figure 1. Confidence bands for $g(x)=\sin (x)$ and $g(x)=x^{3}$ in Model 1 for error variance ratios of $1 / 4$ and $1 / 3$. Gray curves indicate the true function, black solid curves indicate estimates, and dashed curves indicate the $80 \%, 90 \%$, and $95 \%$ confidence bands. 
(a) Men Aged 20-34

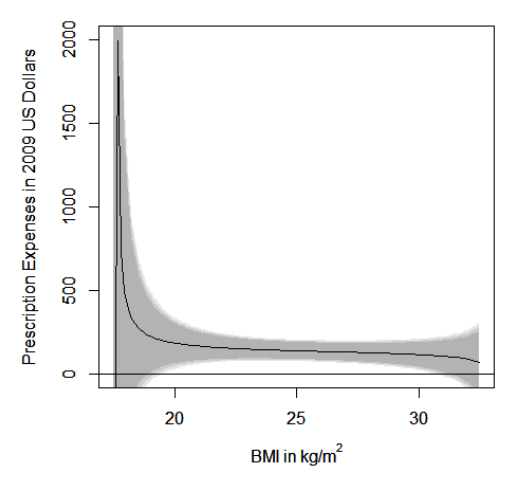

(b) Men Aged 35-49

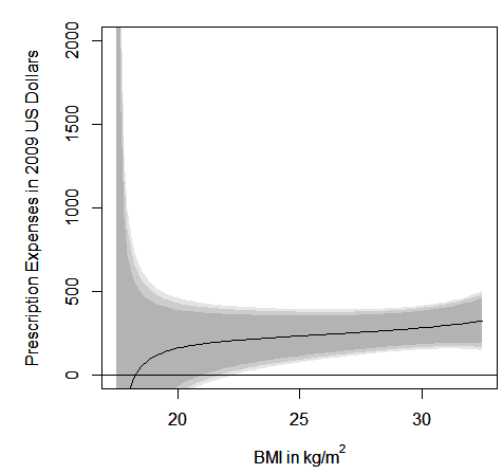

(c) Men Aged 50-64

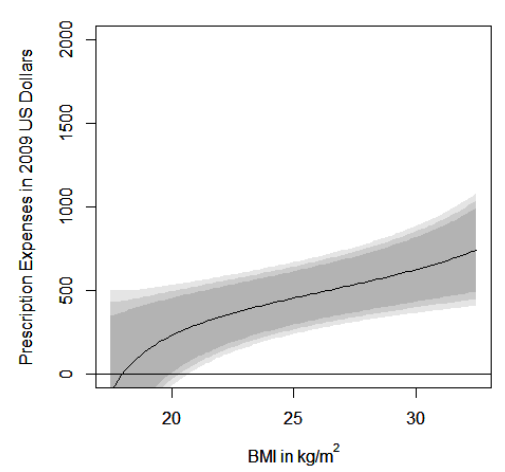

FigURE 2. Estimates and confidence bands for the nonparametric regression of prescription expenses on BMI for (a) men aged from 20 to 34, (b) men aged from 35 to 49, and (c) men aged from 50 to 64 . The horizontal axes measure the BMI in $\mathrm{kg} / \mathrm{m}^{2}$. The vertical axes measure the prescription expenses in 2009 US dollars. The estimates are indicated by solid black curves. The areas shaded by gray-scaled colors indicate $80 \%, 90 \%$, and $95 \%$ confidence bands.

self-report-based BMI $\left(\mathrm{kg} / \mathrm{m}^{2}\right)$ and clinically measured BMI $\left(\mathrm{kg} / \mathrm{m}^{2}\right)$. We denote the former by $W_{j}$ and the latter by $X_{j}$. From the NHANES as a validation data set of size $m$, we can compute $\eta_{j}=W_{j}-X_{j}$ for each $j=1, \ldots, m$. The Panel Survey of Income Dynamics (PSID) provides self-report-based BMI $\left(\mathrm{kg} / \mathrm{m}^{2}\right)$ and prescription expenses. We denote the former by $W_{j}$ and the latter by $Y_{j}$. Additional details of these two data sets can be found in Appendix C.

Combining the NHANES of size $m$ and the PSID of size $n$, we obtain the generated data $\mathcal{D}_{n}=$ $\left\{Y_{1}, \ldots, Y_{n}, W_{1}, \ldots, W_{n}, \eta_{1}, \ldots, \eta_{m}\right\}$ to which we can apply our method to draw confidence bands for the regression function $g$ of the model $Y=g(X)+\epsilon$ with $\mathrm{E}[\epsilon \mid X, U]=0$. We set $I=[15,35]$ as the interval on which we draw confidence bands. The kernel function and the bandwidth rule carry over form our simulation studies. For the sequence $\left\{c_{n}\right\}_{n=1}^{\infty}$ used for bandwidth choice, we follow the recommendation which we made from our simulation results. To account for the different medical conditions across ages, we categorize the sample into the following subsamples: (a) male individuals aged 20-34, (b) male individuals aged 35-49, and (c) male individuals aged 50-64. Details of data processing can be found in Appendix $\mathrm{C}$.

Figure 2 displays estimates and confidence bands for prescription expenses in 2009 US dollars as the dependent variable. The estimates are indicated by solid black curves. The areas shaded by gray-scaled colors indicate $80 \%, 90 \%$, and $95 \%$ confidence bands. The three parts of the figure represent (a) men aged from 20 to 34, (b) men aged from 35 to 49, and (c) men aged from 50 to 64 . We see that the levels of prescription expenses tend to increase in age, as expected. For the groups (a)-(b) of young men, prescription expenses exhibit little partial correlation with BMI. For the group (c) of middle aged men, on the other hand, the relations turn into positive ones. If we look at the $90 \%$ confidence band for the group (c) of men aged from 50 to 64, annual average 
(a) Men Aged 20-34

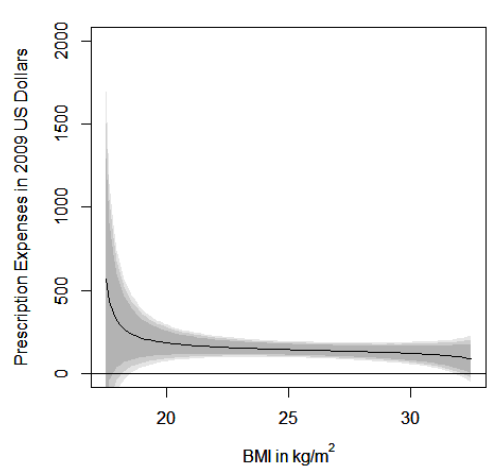

(b) Men Aged 35-49

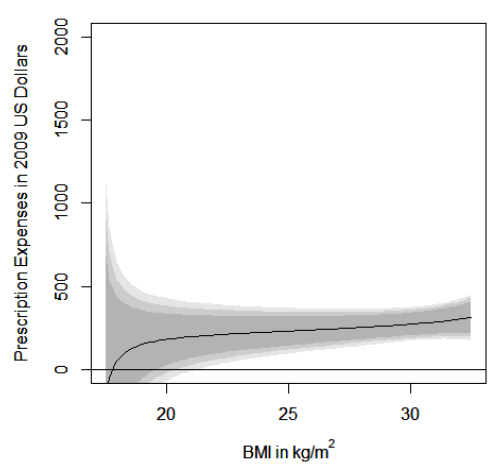

(c) Men Aged 50-64

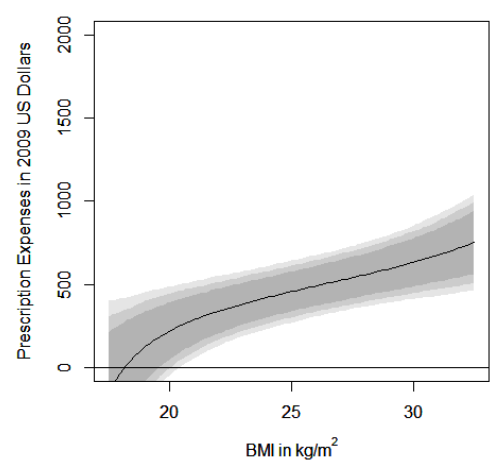

FIGURE 3. Estimates and pointwise confidence intervals for the nonparametric regression of prescription expenses on BMI for (a) men aged from 20 to 34, (b) men aged from 35 to 49 , and (c) men aged from 50 to 64 . The horizontal axes measure the BMI in $\mathrm{kg} / \mathrm{m}^{2}$. The vertical axes measure the prescription expenses in 2009 US dollars. The estimates are indicated by solid black curves. The areas shaded by gray-scaled colors indicate $80 \%, 90 \%$, and $95 \%$ confidence intervals.

prescription expenses are approximately $\$ 0-\$ 495$ if $\mathrm{BMI}=20$, approximately $\$ 266-\$ 642$ if $\mathrm{BMI}$ $=25$, and approximately $\$ 395-\$ 849$ if $\mathrm{BMI}=30$. These concrete numbers illustrate that confidence bands are useful to make interval predictions of incurred average costs, and this convenient feature has practical values added to the existing methods which only allow for reporting estimates with unknown extents of uncertainties. We also present results on total medical expenses in Appendix C.

Finally, we compare the uniform confidence bands and the pointwise confidence intervals in this application, as we did in the simulation studies. Figure 2 displays estimates and pointwise confidence intervals for prescription expenses in 2009 US dollars as the dependent variable. The estimates are indicated by solid black curves. The areas shaded by gray-scaled colors indicate $80 \%$, $90 \%$, and $95 \%$ confidence intervals. The three parts of the figure represent (a) men aged from 20 to 34 , (b) men aged from 35 to 49, and (c) men aged from 50 to 64. Compared with the uniform confidence bands displayed in Figure 2, notice that the pointwise confidence intervals displayed in Figure 2 exhibit somewhat shorter intervals. Given the results of the simulation studies, these pointwise confidence intervals may yield misleading implications about the global shapes of the true regression functions.

\section{EXTENSIONS}

7.1. Application to specification testing. The results of the present paper can be used for specification testing of the regression function $g$. Suppose that we want to test whether the regression function $g$ belongs to a parametric class $\left\{g_{\theta}: \theta \in \Theta\right\}$ where $\Theta$ is a subset of a metric space (in most cases a Euclidean space). Popular specifications of $g$ are polynomials. In cases where $g$ 
is a polynomial, it is possible to estimate the coefficients with $\sqrt{n}$-rate under suitable regularity conditions (Chan and Mak, 1985, Hausman et al., 1991; Cheng and Schneeweiss, 1998). Suppose now that $g=g_{\theta}$ for some $\theta \in \Theta$ and $\theta$ can be estimated by $\widehat{\theta}$ with a sufficiently fast rate, i.e., $\left\|g_{\widehat{\theta}}-g_{\theta}\right\|_{I}=o_{\mathrm{P}}\left\{h_{n}^{-\alpha}\left\{n h_{n} \log \left(1 / h_{n}\right)\right\}^{-1 / 2}\right\}$, and that Assumption 3.1 is satisfied with $g=g_{\theta}$. Then it is not difficult to see from the proof of Theorem 3.2 that

$$
\begin{aligned}
& \widehat{f}_{X}(x) \sqrt{n} h_{n}\left(\widehat{g}(x)-g_{\widehat{\theta}}(x)\right) / \widehat{s}_{n}(x) \\
& \quad=\widehat{f}_{X}(x) \sqrt{n} h_{n}\left(\widehat{g}(x)-g_{\theta}(x)\right) / \widehat{s}_{n}(x)+\widehat{f}_{X}(x) \sqrt{n} h_{n}\left(g_{\theta}(x)-g_{\widehat{\theta}}(x)\right) / \widehat{s}_{n}(x) \\
& \quad=\widehat{f}_{X}(x) \sqrt{n} h_{n}\left(\widehat{g}(x)-g_{\theta}(x)\right) / \widehat{s}_{n}(x)+o_{\mathrm{P}}\left\{\left(\log \left(1 / h_{n}\right)\right)^{-1 / 2}\right\},
\end{aligned}
$$

uniformly in $x \in I$, so that $\mathrm{P}\left\{g_{\widehat{\theta}}(x) \notin \widehat{\mathcal{C}}_{1-\tau}(x)\right.$ for some $\left.x \in I\right\} \rightarrow \tau$. Therefore, the test that rejects the hypothesis that $g=g_{\theta}$ for some $\theta \in \Theta$ if $g_{\widehat{\theta}}(x) \notin \widehat{\mathcal{C}_{1-\tau}}(x)$ for some $x \in I$ is asymptotically of level $\tau$.

The literature on specification testing for EIV regression is large. See Hall and Ma (2007); Song (2008); Otsu and Taylor (2016), and references therein. However, none of those papers considers $L^{\infty}$-based specification tests.

7.2. Additional regressors without measurement errors. In practical applications, we may have additional regressors $Z$, possibly vector valued, without measurement errors. Suppose that we are interested in estimation and making inference on $g(x, z)=\mathrm{E}[Y \mid X=x, Z=z]$. In this section we consider two cases: one is fully nonparametric estimation of the conditional expectation $g(x, z)$, and the other is a partially linear model.

\subsubsection{Fully nonparametric model. Consider a noparametric regression model}

$$
Y=g(X, Z)+\epsilon, W=X+U, \mathrm{E}[\epsilon \mid X, Z, U]=0,
$$

where $Y, X, \epsilon, W$, and $U$ are univariate, $Z$ is $d$-dimensional, and $U$ is independent of $(X, Z)$. We observe $(Y, W, Z)$, but do not observe $X$. Let $\left(Y_{1}, W_{1}, Z_{1}\right), \ldots,\left(Y_{n}, W_{n}, Z_{n}\right)$ be i.i.d. observations on $(Y, W, Z)$, and, as before, we assume that there is an independent sample from the measurement error distribution, $\eta_{1}, \ldots, \eta_{m} \sim f_{U}$ i.i.d. This additional sample may be dependent with $\left(Y_{1}, W_{1}, Z_{1}\right), \ldots,\left(Y_{n}, W_{n}, Z_{n}\right)$.

If $Z$ is finitely distributed, then $g(x, z)$, where $z$ is a mass point, can be estimated by using only observations $j$ for which $Z_{j}=z$. So in what follows we focus on the case where $Z$ is continuous, or more precisely, $(X, Z)$ has a joint density $f_{X Z}(x, z)$. In this case, $g(x, z)$ can be estimated by using observations $j$ for which $Z_{j}$ is "close" to $z$, which can be implemented by using kernel weights. We

will continue to use the same notations $K_{n}(x)$ and $\widehat{K}_{n}(x)$ as defined in Section 2 . Let $L: \mathbb{R}^{d} \rightarrow \mathbb{R}$ be a kernel function (a function that integrates to one) for the $Z$ variable, and consider the estimator $\widehat{g}(x, z)=\widehat{\mu}(x, z) / \widehat{f}_{X Z}(x, z)$, where

$$
\begin{aligned}
& \widehat{\mu}(x, z)=\frac{1}{n h_{n}^{d+1}} \sum_{j=1}^{n} Y_{j} \widehat{K}_{n}\left(\left(x-W_{j}\right) / h_{n}\right) L\left(\left(z-Z_{j}\right) / h_{n}\right), \\
& \widehat{f}_{X Z}(x, z)=\frac{1}{n h_{n}^{d+1}} \sum_{j=1}^{n} \widehat{K}_{n}\left(\left(x-W_{j}\right) / h_{n}\right) L\left(\left(z-Z_{j}\right) / h_{n}\right) .
\end{aligned}
$$


Similarly to the previous case, $\widehat{g}(x, z)-g(x, z)$ can be approximated as $\widehat{g}(x, z)-g(x, z) \approx \frac{1}{f_{X Z}(x, z) n h_{n}^{d+1}} \sum_{j=1}^{n}\left[\left\{Y_{j}-g(x, z)\right\} K_{n}\left(\left(x-W_{j}\right) / h_{n}\right) L\left(\left(z-Z_{j}\right) / h_{n}\right)-A_{n}(x, z)\right]$ with $A_{n}(x, z)=\mathrm{E}\left[\{Y-g(x, z)\} K_{n}\left((x-W) / h_{n}\right) L\left((z-Z) / h_{n}\right)\right]$. So we will modify the multiplier bootstrap process as follows: for $\xi_{1}, \ldots, \xi_{n} \sim N(0,1)$ i.i.d. independent of the data $\mathcal{D}_{n}$, set

$$
\widehat{Z}_{n}^{\xi}(x, z)=\frac{1}{\widehat{s}_{n}(x, z) \sqrt{n}} \sum_{j=1}^{n} \xi_{j}\left\{Y_{j}-\widehat{g}(x, z)\right\} \widehat{K}_{n}\left(\left(x-W_{j}\right) / h_{n}\right) L\left(\left(z-Z_{j}\right) / h_{n}\right),
$$

where $\widehat{s}_{n}(x, z)=\sqrt{\widehat{s}_{n}^{2}(x, z)}$ is defined by

$$
\widehat{s}_{n}^{2}(x, z)=\frac{1}{n} \sum_{j=1}^{n}\left\{Y_{j}-\widehat{g}(x, z)\right\}^{2} \widehat{K}_{n}\left(\left(x-W_{j}\right) / h_{n}\right) L\left(\left(z-Z_{j}\right) / h_{n}\right) .
$$

Let $I \times J$ be a compact rectangle in $\mathbb{R} \times \mathbb{R}^{d}$ on which the joint density $f_{X Z}$ is bounded away from zero, and compute the conditional $(1-\tau)$-quantile of $\left\|\widehat{Z}_{n}^{\xi}\right\|_{I \times J}$ given $\mathcal{D}_{n}$, denoted by $\widehat{c}_{n}(1-\tau)$. Then a confidence confidence band for $g(x, z)$ is given by

$$
\widehat{\mathcal{C}}_{1-\tau}(x, z)=\left[\widehat{g}(x, z) \pm \frac{\widehat{s}_{n}(x, z)}{\widehat{f}_{X Z}(x, z) \sqrt{n} h_{n}^{d+1}} \widehat{c}_{n}(1-\tau)\right],(x, z) \in I \times J .
$$

We make the following assumption, which is analogous to Assumption 3.1. Denote by $f_{W Z}(w, z)$ the joint density of $(W, Z)$. For a multiindex $\alpha=\left(\alpha_{1}, \alpha_{2}, \ldots, \alpha_{d+1}\right)$ (a vector of nonnegative integers), define the differential operator

$$
D^{\alpha}=\frac{\partial^{|\alpha|}}{\partial x^{\alpha_{1}} \partial z_{1}^{\alpha_{2}} \cdots \partial z_{d}^{\alpha_{d+1}}}
$$

with $|\alpha|=\sum_{j=1}^{d+1} \alpha_{j}$. For a given $\beta, B>0$, let $\Sigma_{d+1}(\beta, B)$ denote the class of functions $f:(x, z) \mapsto$ $f(x, z), \mathbb{R} \times \mathbb{R}^{d} \rightarrow \mathbb{R}$ such that $f$ is $k$-times continuously differentiable and

$$
\left|D^{\alpha} f(x, z)-D^{\alpha} f(\bar{x}, \bar{z})\right| \leq B\|(x, z)-(\bar{x}, \bar{z})\|^{\beta-k}, \forall(x, z),(\bar{x}, \bar{z}) \in \mathbb{R} \times \mathbb{R}^{d}
$$

for any multiindex $\alpha=\left(\alpha_{1}, \alpha_{2}, \ldots, \alpha_{d+1}\right)$ with $|\alpha|=k$, where $k$ is the integer such that $k<\beta \leq$ $k+1$. For $t \in \mathbb{R}^{d+1}=\mathbb{R} \times \mathbb{R}^{d}$, we decompose $t$ as $t=\left(t_{1}, t_{-1}\right) \in \mathbb{R} \times \mathbb{R}^{d}$ (we use $\left(t_{1}, t_{-1}\right)$ instead of more precise $\left(t_{1}, t_{-1}^{T}\right)^{T}$ for the sake of notational simplicity).

Assumption 7.1. We assume the following conditions.

(i) $\mathrm{E}\left[Y^{4}\right]<\infty$, the function $w \mapsto \mathrm{E}\left[Y^{2} \mid W=w, Z=z\right] f_{W Z}(w, z)$ is bounded and continuous, and for each $\ell=1,2$, and the function $w \mapsto \mathrm{E}\left[|Y|^{2+\ell} \mid W=w, Z=z\right] f_{W Z}(w, z)$ is bounded.

(ii) The functions $\varphi_{X Z}(t)=\mathrm{E}\left[e^{i\left(t_{1} X+t_{-1}^{T} Z\right)}\right]$ and $\psi_{X Z}(t)=\mathrm{E}\left[g(X, Z) e^{i\left(t_{1} X+t_{-1}^{T} Z\right)}\right]$ for $t=$ $\left(t_{1}, t_{-1}\right) \in \mathbb{R} \times \mathbb{R}^{d}$ are integrable on $\mathbb{R} \times \mathbb{R}^{d}$.

(iii) Condition (iii) in Assumption 3.1 holds.

(iv) The functions $f_{X Z}$ and $g f_{X Z}$ belong to $\Sigma_{d+1}(\beta, B)$ for some $\beta>(d+1) / 2$ and $B>0$. Let $k$ denote the integer such that $k<\beta \leq k+1$. 
(v) Condition ( $v$ ) in Assumption 3.1 is satisfied for the kernel function $K: \mathbb{R} \rightarrow \mathbb{R}$. In addition, let $L: \mathbb{R}^{d} \rightarrow \mathbb{R}$ be a $(k+1)$-th order kernel function such that its Fourier transform $\varphi_{L}\left(t_{-1}\right)$ for $t_{-1} \in \mathbb{R}^{d}$ is supported in $[-1,1]^{d}$, and the function class $\mathcal{L}=\{z \mapsto L(a z+b): a>0, b \in$ $\left.\mathbb{R}^{d}\right\}$ is a VC type class in the sense that, for some positive constants $A$ and $v$,

$$
\sup _{Q} N\left(\mathcal{L},\|\cdot\|_{Q}, \delta\|L\|_{\mathbb{R}}\right) \leq(A / \delta)^{v}, 0<\forall \delta \leq 1
$$

where $\sup _{Q}$ is taken over all finitely discrete distributions on $\mathbb{R}^{d} \cdot{ }^{6}$

(vi) For all $(x, z) \in I \times J, f_{X Z}(x, z)>0$ and $\mathrm{E}\left[\{Y-g(x, z)\}^{2} \mid W=x, Z=z\right] f_{W Z}(x, z)>0$.

(vii) As $n \rightarrow \infty$,

$$
\frac{\left(\log \left(1 / h_{n}\right)\right)^{2}}{(n \wedge m) h_{n}^{2 \alpha+2 d+2}} \rightarrow 0, \frac{n h_{n}^{d+1} \log \left(1 / h_{n}\right)}{m} \rightarrow 0, \text { and } h_{n}^{\alpha+\beta} \sqrt{n h_{n}^{d+1} \log \left(1 / h_{n}\right)} \rightarrow 0 .
$$

Theorem 7.1 (Validity of multiplier bootstrap confidence band). Under Assumption 7.1, we have

$$
\mathrm{P}\left\{g(x, z) \in \widehat{\mathcal{C}}_{1-\tau}(x, z) \forall(x, z) \in I \times J\right\}=1-\tau+o(1)
$$

as $n \rightarrow \infty$. The supremum width of the band $\widehat{\mathcal{C}}_{1-\tau}$ is $O_{\mathrm{P}}\left\{h_{n}^{-\alpha}\left(n h_{n}^{d+1}\right)^{-1 / 2} \sqrt{\log \left(1 / h_{n}\right)}\right\}$.

7.2.2. Partially linear model. Alternatively, we can consider a partially linear model of the form

$$
Y=Z^{T} \beta+g(X)+\epsilon, W=X+U, \mathrm{E}[\epsilon \mid X, Z, U]=0,
$$

where $Z \in \mathbb{R}^{d}$ is observed without measurement errors (observable is $(Y, Z, W)$ ). If we can estimate $\beta$ at a sufficiently fast rate, say, the parametric (i.e., $\sqrt{n}$ ) rate, by some estimator $\widehat{\beta}$, then we can modify the multiplier bootstrap confidence band by replacing $Y_{j}$ with $Y_{j}-Z_{j}^{T} \widehat{\beta}$, and the asymptotic validity of the modified confidence band will go through under similar regularity conditions to those of Theorem 3.2 .

However, obtaining a $\sqrt{n}$-rate estimator for $\beta$ is challenging in the presence of measurement error in $X$. One might be tempted to modify the Robinson (1988) estimator by simply using the deconvolution kernel, but this naive modification does not work. To understand this, assume first that $X$ can be observed; the idea of Robinson (1988) to estimate $\beta$ is 1$)$ to regress $Z$ on $X$ to obtain an estimate of the conditional expectation $m(x)=\mathrm{E}[Z \mid X=x]$ by e.g. a kernel method, and then 2 ) to regress $Y$ on $Z-m(X)$ with $m(\cdot)$ replaced by its estimate. This leads to a $\sqrt{n}$-rate estimator of $\beta$ since

$$
Y=(Z-m(X))^{T} \beta+\left\{m(X)^{T} \beta+g(X)\right\}+\epsilon,
$$

and by construction $Z-m(X)$ is orthogonal to $m(X)^{T} \beta+g(X)$. In the presence of measurement error in $X$, one can estimate the conditional mean $m(x)=\mathrm{E}[Z \mid X=x]$ by using the deconvolution kernel, i.e.,

$$
\widehat{m}(x)=\frac{\left(n h_{n}\right)^{-1} \sum_{j=1}^{n} Z_{j} \widehat{K}_{n}\left(\left(x-W_{j}\right) / h_{n}\right)}{\left(n h_{n}\right)^{-1} \sum_{j=1}^{n} \widehat{K}_{n}\left(\left(x-W_{j}\right) / h_{n}\right)} .
$$

\footnotetext{
${ }^{6}$ For a probability measure $Q$ on a measurable space $(S, \mathcal{S})$ and a class of measurable functions $\mathcal{F}$ on $S$ such that $\mathcal{F} \subset L^{2}(Q)$, let $N\left(\mathcal{F},\|\cdot\|_{Q, 2}, \delta\right)$ denote the $\delta$-covering number for $\mathcal{F}$ with respect to the $L^{2}(Q)$-seminorm $\|\cdot\|_{Q, 2}$.
} 
The problem happens in the second step, because we can not observe $X$ and so there is no way to regress $Y$ on $Z-\widehat{m}(X)$. One could naively use $W$ instead of $X$, but then $Z-\widehat{m}(W) \approx Z-m(W)=$ $Z-\left.\mathrm{E}[Z \mid X=x]\right|_{x=W}$, and so regressing $Y$ on $Z-\widehat{m}(W)$ leads to an inconsistent estimator of $\beta$.

One way to circumvent this fundamental difficulty intrinsic to the measurement error model is to make inference on $g$ without estimating $\beta$. This is possible when $Z$ is finitely discrete. To gain the intuition, suppose first that $Z$ is binary, $Z \in\{0,1\}$. Then the function $g$ can be identified by

$$
g(x)=\mathrm{E}[Y \mid Z=0, X=x]
$$

and so we can estimate $g$ by applying the deonvolution kernel method to the subsample $\left\{j: Z_{j}=0\right\}$, i.e.,

$$
\widehat{g}(x)=\frac{\left(n h_{n}\right)^{-1} \sum_{j=1}^{n} Y_{j} 1\left(Z_{j}=0\right) \widehat{K}_{n}\left(\left(x-W_{j}\right) / h_{n}\right)}{\left(n h_{n}\right)^{-1} \sum_{j=1}^{n} 1\left(Z_{j}=0\right) \widehat{K}_{n}\left(\left(x-W_{j}\right) / h_{n}\right)} .
$$

The multiplier bootstrap confidence band can be constructed by applying the bootstrap process to the subsample $\left\{j: Z_{j}=0\right\}$. It is not hard to see that asymptotic validity of the resulting confidence band follows if the assumption of Theorem 3.2 holds "conditionally on $Z=0$ ".

Consider a more general situation where $Z$ is possibly multidimensional and finitely discrete, i.e., $Z \in\left\{z_{1}, \ldots, z_{L}\right\}$. Assume that there exist weights $\lambda_{1}, \ldots, \lambda_{L} \in \mathbb{R}$ such that $\sum_{\ell=1}^{L} \lambda_{\ell}=1$ and $\sum_{\ell=1}^{L} \lambda_{\ell} z_{\ell}=0$. For instance if $Z \in\{1,2\}$, then we can choose $\lambda_{1}=2$ and $\lambda_{2}=-1$. Such weights need not be unique. Then the function $g$ can be identified by

$$
g(x)=\sum_{\ell=1}^{L} \lambda_{\ell} \mathrm{E}\left[Y \mid Z=z_{\ell}, X=x\right] .
$$

In this case, we can estimate $g(x)$ by

$$
\widehat{g}(x)=\frac{\left(n h_{n}\right)^{-1} \sum_{j=1}^{n} Y_{j} \mathbb{I}\left(Z_{j}\right) \widehat{K}_{n}\left(\left(x-W_{j}\right) / h_{n}\right)}{\left(n h_{n}\right)^{-1} \sum_{j=1}^{n} \mathbb{I}\left(Z_{j}\right) \widehat{K}_{n}\left(\left(x-W_{j}\right) / h_{n}\right)},
$$

where $\mathbb{I}(z)=\sum_{\ell=1}^{L} \lambda_{\ell} 1\left(z=z_{\ell}\right)$. The multiplier bootstrap process should be modified accordingly, and asymptotic validity of the resulting confidence band follows if the assumption of Theorem 3.2 holds conditionally on $Z=z$ for each $z \in\left\{z_{\ell}: \lambda_{\ell}>0\right\}$.

7.3. Confidence bands for conditional distribution functions. The techniques used to derive confidence bands for the conditional mean in EIV regression can be extended to the conditional distribution function. Suppose now that we are interested in constructing confidence bands for the conditional distribution function $g(y, x)=\mathrm{P}(Y \leq y \mid X=x)$ on a compact rectangle $J \times I$ where $J$ and $I$ are compact intervals, and where we do not observe $X$ but instead observe $W=X+U$ with $U$ (measurement error) being independent of $(Y, X)$. As before, we assume that in addition to an independent sample $\left\{\left(Y_{1}, W_{1}\right), \ldots,\left(Y_{n}, W_{n}\right)\right\}$ on $(Y, W)$, there is an independent sample $\left\{\eta_{1}, \ldots, \eta_{m}\right\}$ from the measurement error distribution. Since $g(y, x)=\mathrm{E}[1(Y \leq y) \mid X=x]$ where $1(\cdot)$ denotes the indicator function, we may estimate $g(y, x)$ by $\widehat{g}(y, x)=\widehat{\mu}(y, x) / \widehat{f}_{X}(x)$, where $\widehat{\mu}(y, x)=\left(n h_{n}\right)^{-1} \sum_{j=1}^{n} 1\left(Y_{j} \leq y\right) \widehat{K}_{n}\left(\left(x-W_{j}\right) / h_{n}\right)$. To construct a confidence band for $g(y, x)$, we apply the methodology developed in Section 2 with $Y_{j}$ replaced by $1\left(Y_{j} \leq y\right)$ for each $y$. Let $\widehat{s}_{n}^{2}(y, x)=\frac{1}{n} \sum_{j=1}^{n}\left\{1\left(Y_{j} \leq y\right)-\widehat{g}(y, x)\right\}^{2} \widehat{K}_{n}^{2}\left(\left(x-W_{j}\right) / h_{n}\right)$, and generate independent standard 
normal random variables $\xi_{1}, \ldots, \xi_{n}$ independent of the data $\mathcal{D}_{n}$. Consider the multiplier process $\widehat{Z}_{n}^{\xi}(y, x)=n^{-1 / 2} \sum_{j=1}^{n} \xi_{j}\left\{1\left(Y_{j} \leq y\right)-\widehat{g}(y, x)\right\} \widehat{K}_{n}\left(\left(x-W_{j}\right) / h_{n}\right) / \widehat{s}_{n}(y, x)$, and for $\tau \in(0,1)$, let

$$
\widehat{c}_{n}(1-\tau)=\text { conditional }(1-\tau) \text {-quantile of }\left\|\widehat{Z}_{n}^{\xi}\right\|_{J \times I} \text { given } \mathcal{D}_{n} \text {. }
$$

Then the resulting confidence band for $g(y, x)$ on $J \times I$ is given by

$$
\widehat{\mathcal{C}}_{1-\tau}(y, x)=\left[\widehat{g}(y, x) \pm \frac{\widehat{s}_{n}(y, x)}{\widehat{f}_{X}(x) \sqrt{n} h_{n}} \widehat{c}_{n}(1-\tau)\right],(y, x) \in J \times I .
$$

We make the following assumption, which is analogous to Assumption 3.1.

Assumption 7.2. Let $I, J$ be compact intervals in $\mathbb{R}$. (i) The function $(y, w) \mapsto \mathrm{P}(Y \leq y \mid W=w)$ is continuous in $w$ uniformly in $y \in J$. (ii) The characteristic function of $X, \varphi_{X}(t)=\mathrm{E}\left[e^{i t X}\right], t \in$ $\mathbb{R}$, is integrable on $\mathbb{R}$. Furthermore, $\sup _{y \in J} \int_{\mathbb{R}}\left|\mathrm{E}\left[g(y, X) e^{i t X}\right]\right| d t<\infty$. (iii) Condition (iii) in Assumption 3.1. (iv) The functions $f_{X}$ and $g(y, \cdot) f_{X}(\cdot)$ belong to $\Sigma(\beta, B)$ for some $\beta>1 / 2$ and $B>0$ for all $y \in J$. Let $k$ denote the integer such that $k<\beta \leq k+1$. (v) Condition (v) in Assumption 3.1. (vi) For all $x \in I, f_{X}(x)>0$, and $\inf _{(y, x) \in J \times I} \mathrm{E}\left[\{1(Y \leq y)-g(y, x)\}^{2} \mid W=\right.$ $x] f_{W}(x)>0$. (vii) Condition (vii) in Assumption 3.1.

Theorem 7.2. Under Assumption 7.2. $\mathrm{P}\left\{g(y, x) \in \widehat{\mathcal{C}}_{1-\tau}(y, x) \forall(y, x) \in J \times I\right\} \rightarrow 1-\tau$ as $n \rightarrow \infty$. Furthermore, the supremum width of the band $\widehat{\mathcal{C}}_{1-\tau}$ is $O_{\mathrm{P}}\left\{h_{n}^{-\alpha}\left(n h_{n}\right)^{-1 / 2} \sqrt{\log \left(1 / h_{n}\right)}\right\}$.

Theorem 7.2 is also a new result.

\section{Conclusion}

In this paper, we develop a method to construct uniform confidence bands for nonparametric EIV regression function $g$. We consider the practically relevant case where the distribution of the measurement error is unknown. We assume that there is an independent sample from the measurement error distribution, where the sample from the measurement error distribution need not be independent from the sample on response and predictor variables. Such a sample from the measurement error distribution is available if there is, for example, either 1) validation data or 2) repeated measurements on the latent predictor variable with measurement errors, one of which is symmetrically distributed. We establish asymptotic validity of the proposed confidence band for ordinary smooth measurement error densities, showing that the proposed confidence band contains the true regression function with probability approaching the nominal coverage probability. To the best of our knowledge, this is the first paper to derive asymptotically valid uniform confidence bands for nonparametric EIV regression. We also suggest a two-step SIMEX method for coverageprobability optimal bandwidth choice. Simulation studies verify the finite sample performance of the proposed confidence band. Finally, we discuss extensions of our results to specification testing, cases with additional regressors without measurement errors, and confidence bands for conditional distribution functions.

There are a couple of directions for future research. First, our bootstrap procedure exploits only the first order, and improvements may be possible by exploiting higher orders. For a heuristic investigation, we ran additional simulations of a method that bootstraps $s_{n}(x)$ and $\widehat{\varphi}_{U}(t)$, in 
addition to simulations of our method. These additional simulations turned out exhibit slight improvements for some data generating models. A theoretical investigation of such improvements deserves a topic of future research. Second, as mentioned in Remark 4.1, the data-driven SIMEX bandwidth selection procedure (Delaigle et al. 2015) has not been formally proven to be consistent with theoretical requirements of inference methods even under the simpler settings covered in the existing literature. A theoretical investigation of this practical issue also deserves another topic of future research.

\section{Appendix A. Proofs}

A.1. Technical tools. In this section, we collect technical tools that will be used in the proofs of Theorems 3.1 and 3.2 . The proofs rely on modern empirical process theory. For a probability measure $Q$ on a measurable space $(S, \mathcal{S})$ and a class of measurable functions $\mathcal{F}$ on $S$ such that $\mathcal{F} \subset L^{2}(Q)$, let $N\left(\mathcal{F},\|\cdot\|_{Q, 2}, \delta\right)$ denote the $\delta$-covering number for $\mathcal{F}$ with respect to the $L^{2}(Q)$ seminorm $\|\cdot\|_{Q, 2}$. The class $\mathcal{F}$ is said to be pointwise measurable if there exists a countable subclass $\mathcal{G} \subset \mathcal{F}$ such that for every $f \in \mathcal{F}$ there exists a sequence $g_{m} \in \mathcal{G}$ with $g_{m} \rightarrow f$ pointwise. A function $F: S \rightarrow[0, \infty)$ is said to be an envelope for $\mathcal{F}$ if $F(x) \geq \sup _{f \in \mathcal{F}}|f(x)|$ for all $x \in S$. See Section 2.1 in van der Vaart and Wellner (1996) for details.

Lemma A.1 (A useful maximal inequality). Let $X, X_{1}, \ldots, X_{n}$ be i.i.d. random variables taking values in a measurable space $(S, \mathcal{S})$, and let $\mathcal{F}$ be a pointwise measurable class of (measurable) real-valued functions on $S$ with measurable envelope $F$. Suppose that there exist constants $A \geq e$ and $V \geq 1$ such that

$$
\sup _{Q} N\left(\mathcal{F},\|\cdot\|_{Q, 2}, \delta\|F\|_{Q, 2}\right) \leq(A / \delta)^{V}, 0<\forall \delta \leq 1,
$$

where $\sup _{Q}$ is taken over all finitely discrete distributions on $S$. Furthermore, suppose that $0<$ $\mathrm{E}\left[F^{2}(X)\right]<\infty$, and let $\sigma^{2}>0$ be any positive constant such that $\sup _{f \in \mathcal{F}} \mathrm{E}\left[f^{2}(X)\right] \leq \sigma^{2} \leq$ $\mathrm{E}\left[F^{2}(X)\right]$. Define $B=\sqrt{\mathrm{E}\left[\max _{1 \leq j \leq n} F^{2}\left(X_{j}\right)\right]}$. Then

$$
\begin{aligned}
& \mathrm{E}\left[\left\|\frac{1}{\sqrt{n}} \sum_{j=1}^{n}\left\{f\left(X_{j}\right)-\mathrm{E}[f(X)]\right\}\right\|_{\mathcal{F}}\right] \\
& \quad \leq C\left[\sqrt{V \sigma^{2} \log \left(\frac{A \sqrt{\mathrm{E}\left[F^{2}(X)\right]}}{\sigma}\right)}+\frac{V B}{\sqrt{n}} \log \left(\frac{A \sqrt{\mathrm{E}\left[F^{2}(X)\right]}}{\sigma}\right)\right],
\end{aligned}
$$

where $C>0$ is a universal constant.

Proof. See Corollary 5.1 in Chernozhukov et al. (2014a).

Lemma A.2 (An auxiliary maximal inequality). Let $\zeta_{1}, \ldots, \zeta_{n}$ be random variables such that $\mathrm{E}\left[\left|\zeta_{j}\right|^{r}\right]<\infty$ for all $j=1, \ldots, n$ for some $r \geq 1$. Then

$$
\mathrm{E}\left[\max _{1 \leq j \leq n}\left|\zeta_{j}\right|\right] \leq n^{1 / r} \max _{1 \leq j \leq n}\left(\mathrm{E}\left[\left|\zeta_{j}\right|^{r}\right]\right)^{1 / r}
$$


Proof. This inequality is well known, and follows from Jensen's inequality. Indeed, $\mathrm{E}\left[\max _{1 \leq j \leq n}\left|\zeta_{j}\right|\right] \leq$ $\left(\mathrm{E}\left[\max _{1 \leq j \leq n}\left|\zeta_{j}\right|^{r}\right]\right)^{1 / r} \leq\left(\sum_{j=1}^{n} \mathrm{E}\left[\left|\zeta_{j}\right|^{r}\right]\right)^{1 / r} \leq n^{1 / r} \max _{1 \leq j \leq n}\left(\mathrm{E}\left[\left|\zeta_{j}\right|^{r}\right]\right)^{1 / r}$.

The following anti-concentration inequality for the supremum of a Gaussian process will play a crucial role in the proofs of Theorems 3.1 and 3.2 .

Lemma A.3 (Anti-concentration for the supremum of a Gaussian process). Let $T$ be a nonempty set, and let $X=\left(X_{t}: t \in T\right)$ be a tight Gaussian random variable in $\ell^{\infty}(T)$ with mean zero and $\mathrm{E}\left[X_{t}^{2}\right]=1$ for all $t \in T$. Then for any $h>0$,

$$
\sup _{x \in \mathbb{R}} \mathrm{P}\left\{\left|\|X\|_{T}-x\right| \leq h\right\} \leq 4 h\left(1+\mathrm{E}\left[\|X\|_{T}\right]\right) .
$$

Proof. See Corollary 2.1 in Chernozhukov et al. (2014b); see also Theorem 3 in Chernozhukov et al. (2015).

A.2. Proof of Theorem 3.1. In what follows, we always assume Assumption 3.1. We first prove some preliminary lemmas. Recall that $A_{n}(x)=\mathrm{E}\left[\{Y-g(x)\} K_{n}\left((x-W) / h_{n}\right)\right]$ and $s_{n}^{2}(x)=$ $\operatorname{Var}\left(\{Y-g(x)\} K_{n}\left((x-W) / h_{n}\right)\right)$. Observe that $\left\|K_{n}\right\|_{\mathbb{R}}=O\left(h_{n}^{-\alpha}\right)$ under our assumption. In what follows, the notation $\lesssim$ signifies that the left hand side is bounded by the right hand side up to a positive constant independent of $n$ and $x$.

Lemma A.4. The following bounds hold: (i) $\left\|A_{n}\right\|_{I}=O\left(h_{n}^{\beta+1}\right)$. (ii) For sufficiently large $n$, $\inf _{x \in I} s_{n}^{2}(x) \gtrsim h_{n}^{-2 \alpha+1}$. (iii) For $\ell=0,1,2$, we have $\sup _{x \in \mathbb{R}} \mathrm{E}\left[\left|Y K_{n}\left((x-W) / h_{n}\right)\right|^{2+\ell}\right]=O\left(h_{n}^{-(2+\ell) \alpha+1}\right)$.

Proof. (i). Since $\mathrm{E}\left[Y e^{i t W}\right]=\mathrm{E}\left[\{g(X)+U\} e^{i t(X+U)}\right]=\psi_{X}(t) \varphi_{U}(t)$,

$$
\mathrm{E}\left[Y K_{n}\left((x-W) / h_{n}\right)\right]=\frac{h_{n}}{2 \pi} \int_{\mathbb{R}} e^{-i t x} \psi_{X}(t) \varphi_{K}\left(t h_{n}\right) d t .
$$

Since $\psi_{X}(\cdot)$ and $\varphi_{K}\left(\cdot h_{n}\right)$ are the Fourier transforms of $g f_{X}$ and $h_{n}^{-1} K\left(\cdot / h_{n}\right)$, respectively, the Fourier inversion formula yields that

$$
\frac{h_{n}}{2 \pi} \int_{\mathbb{R}} e^{-i t x} \psi_{X}(t) \varphi_{K}\left(t h_{n}\right) d t=\int_{\mathbb{R}} g(w) f_{X}(w) K\left((x-w) / h_{n}\right) d w .
$$

Note that the far left and right hand sides are continuous in $x$, and so the equality holds for all $x \in \mathbb{R}$. Likewise, we have $\mathrm{E}\left[K_{n}\left((x-W) / h_{n}\right)\right]=\int_{\mathbb{R}} f_{X}(w) K\left((x-w) / h_{n}\right) d w$ for all $x \in \mathbb{R}$, so that

$$
A_{n}(x)=h_{n} \int_{\mathbb{R}}\left\{g\left(x-h_{n} w\right)-g(x)\right\} f_{X}\left(x-h_{n} w\right) K(w) d w .
$$

By the Taylor expansion, for any $x, w \in \mathbb{R}$,

$$
\begin{gathered}
\left\{g\left(x-h_{n} w\right)-g(x)\right\} f_{X}\left(x-h_{n} w\right)=\sum_{j=0}^{k-1} \frac{\left(g f_{X}\right)^{(j)}(x)-g(x) f_{X}^{(j)}(x)}{j !}\left(-h_{n} w\right)^{j} \\
+\frac{\left(g f_{X}\right)^{(k)}\left(x-\theta h_{n} w\right)-g(x) f_{X}^{(k)}\left(x-\theta h_{n} w\right)}{k !}\left(-h_{n} w\right)^{k}
\end{gathered}
$$


for some $\theta \in[0,1]$. Since $\int_{\mathbb{R}} w^{j} K(w) d w=0$ for $j=1, \ldots, k$ and $f_{X}, g f_{X} \in \Sigma(\beta, B)$, we have

$$
\begin{aligned}
\left|A_{n}(x)\right|= & h_{n} \mid \int_{\mathbb{R}}\left[\left\{g\left(x-h_{n} w\right)-g(x)\right\} f_{X}\left(x-h_{n} w\right)\right. \\
& \left.-\frac{\left(g f_{X}\right)^{(k)}(x)-g(x) f_{X}^{(k)}(x)}{k !}\left(-h_{n} w\right)^{k}\right] K(w) d w \mid \\
\leq & \frac{\left(1+\|g\|_{I}\right) B h_{n}^{\beta+1}}{k !} \int_{\mathbb{R}}|w|^{\beta}|K(w)| d w .
\end{aligned}
$$

This shows that $\left\|A_{n}\right\|_{I}=O\left(h_{n}^{\beta+1}\right)$.

(ii). Since $A_{n}(x)=\mathrm{E}\left[\{Y-g(x)\} K_{n}\left((x-W) / h_{n}\right)\right]=O\left(h_{n}^{\beta+1}\right)$ uniformly in $x \in I$, it suffices to show that $\inf _{x \in I} \mathrm{E}\left[\{Y-g(x)\}^{2} K_{n}^{2}\left((x-W) / h_{n}\right)\right] \gtrsim(1-o(1)) h_{n}^{-2 \alpha+1}$. We first verify that

$$
\mathrm{E}[Y \mid W=w] f_{W}(w)=\left(\left(g f_{X}\right) * f_{U}\right)(w) .
$$

To this end, it is enough to verify that the Fourier transforms of both sides agree. The Fourier transform of the right hand is $\psi_{X}(t) \varphi_{U}(t)$. On the other hand, the Fourier transform of the left hand side is

$$
\begin{aligned}
\mathrm{E}\left[\mathrm{E}[Y \mid W] e^{i t W}\right] & =\mathrm{E}\left[\mathrm{E}[\underbrace{\mathrm{E}[Y \mid X, U]}_{=g(X)} \mid W] e^{i t W}\right]=\mathrm{E}\left[\mathrm{E}[g(X) \mid W] e^{i t W}\right]=\mathrm{E}\left[g(X) e^{i t W}\right] \\
& =\mathrm{E}\left[g(X) e^{i t(X+U)}\right]=\mathrm{E}\left[g(X) e^{i t X}\right] \mathrm{E}\left[e^{i t U}\right]=\psi_{X}(t) \varphi_{U}(t) .
\end{aligned}
$$

Define

$$
\begin{aligned}
V(x, w) & =\mathrm{E}\left[\{Y-g(x)\}^{2} \mid W=w\right] f_{W}(w) \\
& =\left(\mathrm{E}\left[Y^{2} \mid W=w\right]+g^{2}(x)\right) f_{W}(w)-2 g(x)\left(\left(g f_{X}\right) * f_{\varepsilon}\right)(w) .
\end{aligned}
$$

The function $\left(g f_{X}\right) * f_{\varepsilon}$ is bounded and continuous by boundedness of $g f_{X}$. Since $\mathrm{E}\left[Y^{2} \mid W=\right.$ · ], $f_{W}$, and $\left(g f_{X}\right) * f_{\varepsilon}$ are bounded and continuous on $\mathbb{R}$, and $g$ is bounded and continuous on $I$, we have that the function $(x, w) \mapsto V(x, w)$ is bounded and continuous on $I \times \mathbb{R}$. In particular, since $V(x, x)>0$ for all $x \in I$ under our assumption, we have that $\inf _{x \in I} V(x, x)>0$.

Now, observe that

$$
\begin{aligned}
\mathrm{E}\left[\{Y-g(x)\}^{2} K_{n}^{2}\left((x-W) / h_{n}\right)\right] & =\int_{\mathbb{R}} V(x, w) K_{n}^{2}\left((x-w) / h_{n}\right) d w \\
& =h_{n} \int_{\mathbb{R}} V\left(x, x-h_{n} w\right) K_{n}^{2}(w) d w .
\end{aligned}
$$

Furthermore, we have that

$$
\int_{\mathbb{R}} K_{n}^{2}(w) d w=\frac{1}{2 \pi} \int_{\mathbb{R}} \frac{\left|\varphi_{K}(t)\right|^{2}}{\left|\varphi_{\varepsilon}\left(t / h_{n}\right)\right|^{2}} d t \sim h_{n}^{-2 \alpha}
$$

by Plancherel's theorem. Hence, it suffices to show that

$$
\sup _{x \in I}\left|h_{n}^{2 \alpha} \int_{\mathbb{R}}\left\{V\left(x, x-h_{n} w\right)-V(x, x)\right\} K_{n}^{2}(w) d w\right| \rightarrow 0 .
$$


From the proof of Lemma 3 in Kato and Sasaki (2016), we have that $h_{n}^{2 \alpha} K_{n}^{2}(x) \lesssim \min \left\{1, x^{-2}\right\}$. By the definition of $V(x, w)$, for any $\rho>0$, there exists sufficiently small $\delta>0$ such that $\mid V(x, x+$ $w)-V(x, x) \mid \leq \rho$ for all $x \in I$ whenever $|w| \leq \delta$. Therefore,

$$
\begin{aligned}
& \sup _{x \in I} \int_{\mathbb{R}}\left|V\left(x, x-h_{n} w\right)-V(x, x)\right| h_{n}^{2 \alpha} K_{n}^{2}(w) d w \\
& \quad \lesssim \rho \int_{|w| \leq \delta / h_{n}} \min \left\{1, w^{-2}\right\} d w+2\|V\|_{I \times \mathbb{R}} \int_{|w|>\delta / h_{n}} w^{-2} d w \lesssim \rho+o(1) .
\end{aligned}
$$

(iii). Pick any $\ell=0,1,2$. Since $\left\|K_{n}\right\|_{\mathbb{R}} \lesssim h_{n}^{-\alpha}$, we have

$$
\begin{aligned}
\mathrm{E}\left[\left|Y K_{n}\left((x-W) / h_{n}\right)\right|^{2+\ell}\right] & =h_{n} \int_{\mathbb{R}} \mathrm{E}\left[|Y|^{2+\ell} \mid W=x-h_{n} w\right]\left|K_{n}(w)\right|^{2+\ell} f_{W}\left(x-h_{n} w\right) d w \\
& \lesssim h_{n}^{-\ell \alpha+1} \int_{\mathbb{R}} V_{\ell}\left(x-h_{n} w\right) K_{n}^{2}(w) d w \\
& \leq h_{n}^{-\ell \alpha+1}\left\|V_{\ell}\right\|_{\mathbb{R}} \int_{\mathbb{R}} K_{n}^{2}(w) d w \lesssim h_{n}^{-(2+\ell) \alpha+1},
\end{aligned}
$$

where $V_{\ell}(w)=\mathrm{E}\left[|Y|^{2+\ell} \mid W=w\right] f_{W}(w)$. This completes the proof.

Lemma A.5. $\left\|\widehat{\varphi}_{U}-\varphi_{U}\right\|_{\left[-h_{n}^{-1}, h_{n}^{-1}\right]}=O_{\mathrm{P}}\left\{m^{-1 / 2} \log \left(1 / h_{n}\right)\right\}$.

Proof. See Lemma 4 in Kato and Sasaki (2016); see also Theorem 4.1 in Neumann and Reiß (2009).

Consider the following classes of functions

$$
\left\{\begin{array}{l}
\mathcal{F}_{n}^{(1)}=\left\{(y, w) \mapsto y K_{n}\left((x-w) / h_{n}\right): x \in \mathbb{R}\right\}, \\
\mathcal{F}_{n}^{(2)}=\left\{(y, w) \mapsto \frac{1}{s_{n}(x)}\{y-g(x)\} K_{n}\left((x-w) / h_{n}\right): x \in I\right\}, \\
\mathcal{F}_{n}^{(3)}=\left\{(y, w) \mapsto\{y-g(x)\} K_{n}^{2}\left((x-w) / h_{n}\right): x \in I\right\}, \\
\mathcal{F}_{n}^{(4)}=\left\{(y, w) \mapsto \frac{1}{s_{n}^{2}(x)}\{y-g(x)\}^{2} K_{n}^{2}\left((x-w) / h_{n}\right): x \in I\right\} .
\end{array}\right.
$$

In view of the fact that $\left\|K_{n}\right\|_{\mathbb{R}} \lesssim h_{n}^{-\alpha}$ and $\inf _{x \in I} s_{n}(x) \gtrsim h_{n}^{-\alpha+1 / 2}$, choose constants $D_{1}, D_{2}>$ 0 (independent of $n$ ) such that $\left\|K_{n}\right\|_{\mathbb{R}} \leq D_{1} h_{n}^{-\alpha}$ and $\left\|1 / s_{n}\right\|_{I} \leq D_{2} h_{n}^{\alpha-1 / 2}$. Let $F_{n}^{(1)}(y, w)=$ $D_{1}|y| h_{n}^{-\alpha}, F_{n}^{(2)}(y, w)=D_{1} D_{2}\left(|y|+\|g\|_{I}\right) / \sqrt{h_{n}}, F_{n}^{(3)}(y, w)=D_{1}\left(|y|+\|g\|_{I}\right) h_{n}^{-2 \alpha}$, and $F_{n}^{(4)}(y, w)=$ $\left\{F_{n}^{(2)}(y, w)\right\}^{2}$. Note that $F_{n}^{(\ell)}$ is an envelope function for $\mathcal{F}_{n}^{(\ell)}$ for each $\ell=1, \ldots, 4$.

Lemma A.6. There exist constants $A, v \geq e$ independent of $n$ such that

$$
\sup _{Q} N\left(\mathcal{F}_{n}^{(\ell)},\|\cdot\|_{Q, 2}, \delta\left\|F_{n}^{(\ell)}\right\|_{Q, 2}\right) \leq(A / \delta)^{v}, 0<\forall \delta \leq 1, \ell=1, \ldots, 4
$$

where $\sup _{Q}$ is taken over all finitely discrete distributions on $\mathbb{R}^{2}$.

Proof. Consider the following classes of functions: $\mathcal{K}_{n}=\left\{w \mapsto K_{n}\left((x-w) / h_{n}\right): x \in \mathbb{R}\right\}$ and $\mathcal{K}_{n}^{2}=\left\{f^{2}: f \in \mathcal{K}_{n}\right\}$. Lemma 1 in Kato and Sasaki (2016) and Corollary A.1 in Chernozhukov et al. (2014a) yield that there exist constants $A_{1}, v_{1} \geq e$ independent of $n$ such that $\sup _{Q} N\left(\mathcal{K}_{n}, \|\right.$. $\left.\|_{Q, 2}, D_{1} h_{n}^{-\alpha} \delta\right) \leq\left(A_{1} / \delta\right)^{v_{1}}$ and $\sup _{Q} N\left(\mathcal{K}_{n}^{2},\|\cdot\|_{Q, 2}, D_{1}^{2} h_{n}^{-2 \alpha} \delta\right) \leq\left(A_{1} / \delta\right)^{v_{1}}$ for all $0<\delta \leq 1$.

In what follows, we only prove 15 for $\ell=2$; the proofs for the other cases are completely analogous given the above bounds on the covering numbers for $\mathcal{K}_{n}$ and $\mathcal{K}_{n}^{2}$. Let $\mathcal{H}_{n}=\{y \mapsto$ 
$\left.\{y-g(x)\} / s_{n}(x): x \in I\right\}$, and observe that, since $\left\|1 / s_{n}\right\|_{I} \leq D_{2} h_{n}^{\alpha-1 / 2}$, there exist constants $A_{2}, v_{2} \geq e$ independent of $n$ such that $\sup _{Q} N\left(\mathcal{H}_{n},\|\cdot\|_{Q, 2}, \delta\left\|H_{n}\right\|_{Q, 2}\right) \leq\left(A_{2} / \delta\right)^{v_{2}}$ for all $0<\delta \leq 1$, where $H_{n}(y)=D_{2}\left(|y|+\|g\|_{I}\right) h_{n}^{\alpha-1 / 2}$ is an envelope function for $\mathcal{H}_{n}$. This can be verified by a direct calculation, or observing that $\mathcal{H}_{n}(\subset\{y \mapsto a y+b: a>0, b \in \mathbb{R}\})$ is a VC subgraph class with VC index at most 4 (cf. van der Vaart and Wellner, 1996, Lemma 2.6.15), and applying Theorem 2.6.7 in van der Vaart and Wellner (1996). Let $\mathcal{H}_{n} \mathcal{K}_{n}:=\left\{(y, w) \mapsto f_{1}(y) f_{2}(w): f_{1} \in \mathcal{H}_{n}, f_{2} \in \mathcal{K}_{n}\right\} \supset \mathcal{F}_{n}^{(2)}$, and note that $H_{n}(y) D_{1} h_{n}^{-\alpha}=F_{n}^{(2)}(y, w)$. From Corollary A.1 in Chernozhukov et al. (2014a), there exist constants $A_{3}, v_{3} \geq e$ independent of $n \operatorname{such}$ that $\sup _{Q} N\left(\mathcal{H}_{n} \mathcal{K}_{n},\|\cdot\|_{Q, 2}, \delta\left\|F_{n}^{(2)}\right\|_{Q, 2}\right) \leq\left(A_{3} / \delta\right)^{v_{3}}$ for all $0<\delta \leq 1$. Now, the desired result follows from the observation that $N\left(\mathcal{F}_{n}^{(2)},\|\cdot\|_{Q, 2}, 2 \delta\right) \leq$ $N\left(\mathcal{H}_{n} \mathcal{K}_{n},\|\cdot\|_{Q, 2}, \delta\right)$ for all $\delta>0$.

Lemma A.7. We have

$$
\begin{aligned}
& \left\|\widehat{f}_{X}^{*}(\cdot)-\mathrm{E}\left[\widehat{f}_{X}^{*}(\cdot)\right]\right\|_{\mathbb{R}}=O_{\mathrm{P}}\left\{h_{n}^{-\alpha}\left(n h_{n}\right)^{-1 / 2} \sqrt{\log \left(1 / h_{n}\right)}\right\}, \\
& \left\|\mathrm{E}\left[\widehat{f}_{X}^{*}(\cdot)\right]-f_{X}(\cdot)\right\|_{\mathbb{R}}=O\left(h_{n}^{\beta}\right)=o\left\{h_{n}^{-\alpha}\left(n h_{n} \log \left(1 / h_{n}\right)\right)^{-1 / 2}\right\},
\end{aligned}
$$

and $\left\|\widehat{\mu}^{*}(\cdot)-\mathrm{E}\left[\widehat{\mu}^{*}(\cdot)\right]\right\|_{\mathbb{R}}=O_{\mathrm{P}}\left\{h_{n}^{-\alpha}\left(n h_{n}\right)^{-1 / 2} \sqrt{\log \left(1 / h_{n}\right)}\right\}$.

Proof. The first two results are implicit in the proofs of Corollaries 1 and 2 in Kato and Sasaki (2016). To prove the last result, we shall apply Lemma A.1 to the class of functions $\mathcal{F}_{n}^{(1)}$. From Lemma A.4 (iii), we have that $\sup _{x \in \mathbb{R}} \mathrm{E}\left[Y^{2} K_{n}^{2}\left((x-W) / h_{n}\right)\right]=O\left(h_{n}^{-2 \alpha+1}\right)$. In view of the covering number bound for $\mathcal{F}_{n}^{(1)}$ given in Lemma A.6, we may apply Lemma A.1 to $\mathcal{F}_{n}^{(1)}$ to conclude that

$$
\begin{aligned}
& \left(n h_{n}\right) \mathrm{E}\left[\left\|\widehat{\mu}^{*}(\cdot)-\mathrm{E}\left[\widehat{\mu}^{*}(\cdot)\right]\right\|_{\mathbb{R}}\right]=\mathrm{E}\left[\left\|\sum_{j=1}^{n}\left\{f\left(Y_{j}, W_{j}\right)-\mathrm{E}[f(Y, W)]\right\}\right\|_{\mathcal{F}^{(1)}}\right] \\
& \lesssim h_{n}^{-\alpha} \sqrt{n h_{n} \log \left(1 / h_{n}\right)}+h_{n}^{-\alpha} \sqrt{\mathrm{E}\left[\max _{1 \leq j \leq n} Y_{j}^{2}\right]} \log \left(1 / h_{n}\right) .
\end{aligned}
$$

From Lemma A.2, we have $\mathrm{E}\left[\max _{1 \leq j \leq n} Y_{j}^{2}\right]=O\left(n^{1 / 2}\right)$, so that we have

$$
\left(n h_{n}\right) \mathrm{E}\left[\left\|\widehat{\mu}^{*}(\cdot)-\mathrm{E}\left[\widehat{\mu}^{*}(\cdot)\right]\right\|_{\mathbb{R}}\right] \lesssim h_{n}^{-\alpha} \sqrt{n h_{n} \log \left(1 / h_{n}\right)}+h_{n}^{-\alpha} n^{1 / 4} \log \left(1 / h_{n}\right),
$$

and the right hand side is $\lesssim h_{n}^{-\alpha} \sqrt{n h_{n} \log \left(1 / h_{n}\right)}$ from the first condition in 12 . This completes the proof.

We are now in position to prove Theorem 3.1 .

Proof of Theorem 3.1. We divide the proof into two steps.

Step 1. Let $r_{n}=h_{n}^{-\alpha}\left\{n h_{n} \log \left(1 / h_{n}\right)\right\}^{-1 / 2}$. We first prove that

$$
\widehat{g}(x)-g(x)=\frac{1}{f_{X}(x)} \frac{1}{n h_{n}} \sum_{j=1}^{n}\left[\left\{Y_{j}-g(x)\right\} K_{n}\left(\left(x-W_{j}\right) / h_{n}\right)-A_{n}(x)\right]+o_{\mathrm{P}}\left(r_{n}\right)
$$

uniformly in $x \in I$.

To this end, we shall show that $\left\|\widehat{\mu}-\widehat{\mu}^{*}\right\|_{\mathbb{R}}=o_{\mathrm{P}}\left(r_{n}\right)$. First, observe from Lemma A.5 that

$$
\inf _{|t| \leq h_{n}^{-1}}\left|\widehat{\varphi}_{U}(t)\right| \geq \inf _{|t| \leq h_{n}^{-1}}\left|\varphi_{U}(t)\right|-O_{\mathrm{P}}\left\{m^{-1 / 2} \log \left(1 / h_{n}\right)\right\} \gtrsim\left(1-o_{\mathrm{P}}(1)\right) h_{n}^{\alpha} .
$$


Let $\psi_{Y W}(t)=\mathrm{E}\left[Y e^{i t W}\right]=\mathrm{E}\left[\{g(X)+\epsilon\} e^{i t(X+U)}\right]=\psi_{X}(t) \varphi_{U}(t)$, and let $\widehat{\psi}_{Y W}(t)=n^{-1} \sum_{j=1}^{n} Y_{j} e^{i t W_{j}}$. Decomposing the integral over $\mathbb{R}$ into $\int_{\left\{\psi_{X} \neq 0\right\}}$ and $\int_{\left\{\psi_{X}=0\right\}}$, we obtain the following identity for $\widehat{\mu}(x)-\widehat{\mu}^{*}(x):$

$$
\begin{aligned}
& \widehat{\mu}(x)-\widehat{\mu}^{*}(x) \\
& =\frac{1}{2 \pi} \int_{\left\{\psi_{X} \neq 0\right\}} e^{-i t x} \varphi_{K}\left(t h_{n}\right)\left\{\frac{\widehat{\psi}_{Y W}(t)}{\psi_{Y W}(t)}-1\right\}\left\{\frac{\varphi_{U}(t)}{\widehat{\varphi}_{U}(t)}-1\right\} \psi_{X}(t) d t \\
& \quad+\frac{1}{2 \pi} \int_{\left\{\psi_{X}=0\right\}} e^{-i t x} \frac{\varphi_{K}\left(t h_{n}\right)}{\varphi_{U}(t)} \widehat{\psi}_{Y W}(t)\left\{\frac{\varphi_{U}(t)}{\widehat{\varphi}_{U}(t)}-1\right\} d t \\
& \quad+\frac{1}{2 \pi} \int_{\mathbb{R}} e^{-i t x} \varphi_{K}\left(t h_{n}\right)\left\{\frac{\varphi_{U}(t)}{\widehat{\varphi}_{U}(t)}-1\right\} \psi_{X}(t) d t .
\end{aligned}
$$

Hence the Cauchy-Schwarz inequality yields that

$$
\begin{aligned}
& \left|\widehat{\mu}(x)-\widehat{\mu}^{*}(x)\right|^{2} \\
& \lesssim\left\{\int_{\left\{\psi_{X} \neq 0\right\} \cap\left[-h_{n}^{-1}, h_{n}^{-1}\right]}\left|\frac{\widehat{\psi}_{Y W}(t)}{\psi_{Y W}(t)}-1\right|^{2}\left|\psi_{X}(t)\right|^{2} d t\right\}\left\{\int_{-h_{n}^{-1}}^{h_{n}^{-1}}\left|\frac{\varphi_{U}(t)}{\widehat{\varphi}_{U}(t)}-1\right|^{2} d t\right\} \\
& \quad+h_{n}^{-2 \alpha}\left\{\int_{\left\{\psi_{X}=0\right\} \cap\left[-h_{n}^{-1}, h_{n}^{-1}\right]}\left|\widehat{\psi}_{Y W}(t)\right|^{2} d t\right\}\left\{\int_{-h_{n}^{-1}}^{h_{n}^{-1}}\left|\frac{\varphi_{U}(t)}{\widehat{\varphi}_{U}(t)}-1\right|^{2} d t\right\} \\
& \quad+\int_{-h_{n}^{-1}}^{h_{n}^{-1}}\left|\frac{\varphi_{U}(t)}{\widehat{\varphi}_{U}(t)}-1\right|^{2}\left|\psi_{X}(t)\right| d t .
\end{aligned}
$$

We shall bound each term on the right hand side. Observe that

$$
\int_{-h_{n}^{-1}}^{h_{n}^{-1}}\left|\frac{\varphi_{U}(t)}{\widehat{\varphi}_{U}(t)}-1\right|^{2} d t \leq O_{\mathrm{P}}\left(h_{n}^{-2 \alpha}\right) \int_{-h_{n}^{-1}}^{h_{n}^{-1}}\left|\varphi_{U}(t)-\widehat{\varphi}_{U}(t)\right|^{2} d t
$$

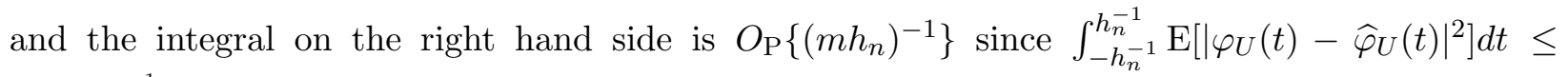
$m^{-1} \int_{-h_{n}^{-1}}^{h_{n}^{-1}} d t=2\left(m h_{n}\right)^{-1}$. Likewise, using the fact that $\psi_{X}$ is integrable, we have that the last term on the right hand side of $(16)$ is $O_{\mathrm{P}}\left(h_{n}^{-2 \alpha} m^{-1}\right)$. For any $t \in \mathbb{R}$ with $\psi_{X}(t) \neq 0$, we have $\mathrm{E}\left[\left|\widehat{\psi}_{Y W}(t) / \psi_{Y W}(t)-1\right|^{2}\right] \leq \mathrm{E}\left[Y^{2}\right] /\left\{n\left|\psi_{Y W}(t)\right|^{2}\right\}$, so that

$$
\begin{gathered}
\mathrm{E}\left[\int_{\left\{\psi_{X} \neq 0\right\} \cap\left[-h_{n}^{-1}, h_{n}^{-1}\right]}\left|\frac{\widehat{\psi}_{Y W}(t)}{\psi_{Y W}(t)}-1\right|^{2}\left|\psi_{X}(t)\right|^{2} d t\right] \\
\lesssim n^{-1} \int_{-h_{n}^{-1}}^{h_{n}^{-1}} \frac{1}{\left|\varphi_{U}(t)\right|^{2}} d t \lesssim h_{n}^{-2 \alpha}\left(n h_{n}\right)^{-1} .
\end{gathered}
$$

Finally, for any $t \in \mathbb{R}$ with $\psi_{X}(t)=0$, we have $\psi_{Y W}(t)=0$, so that

$$
\mathrm{E}\left[\int_{\left\{\psi_{X}=0\right\} \cap\left[-h_{n}^{-1}, h_{n}^{-1}\right]}\left|\widehat{\psi}_{Y W}(t)\right|^{2} d t\right] \lesssim\left(n h_{n}\right)^{-1} .
$$

Therefore, we have $\left\|\widehat{\mu}-\widehat{\mu}^{*}\right\|_{\mathbb{R}}^{2}=O_{\mathrm{P}}\left(h_{n}^{-4 \alpha-2} n^{-1} m^{-1}+h_{n}^{-2 \alpha} m^{-1}\right)=o_{\mathrm{P}}\left(r_{n}^{2}\right)$. 
From Step 2 in the proof of Theorem 1 of Kato and Sasaki $(2016)$, it follows that $\left\|\widehat{f}_{X}-\widehat{f}_{X}^{*}\right\|_{\mathbb{R}}=$ $o_{\mathrm{P}}\left(r_{n}\right)$, which in particular implies that $\left\|\widehat{f}_{X}-f_{X}\right\|_{I} \leq\left\|\widehat{f}_{X}-\widehat{f}_{X}^{*}\right\|_{I}+\left\|\widehat{f}_{X}^{*}-f_{X}\right\|_{I}=o_{P}(1)$ so that $\left\|1 / \widehat{f}_{X}\right\|_{I}=O_{\mathrm{P}}(1)$. Furthermore, $\left\|\widehat{\mu}^{*}\right\|_{I} \leq\left\|\mathrm{E}\left[\widehat{\mu}^{*}(\cdot)\right]\right\|_{I}+\left\|\widehat{\mu}^{*}(\cdot)-\mathrm{E}\left[\widehat{\mu}^{*}(\cdot)\right]\right\|_{I} \lesssim \int_{\mathbb{R}}\left|\psi_{X}(t)\right| d t+o_{\mathrm{P}}(1)=$ $O_{\mathrm{P}}(1)$. Therefore,

$$
\begin{aligned}
\left\|\widehat{g}-\widehat{g}^{*}\right\|_{I} & \leq\left\|1 / \widehat{f}_{X}\right\|_{I}\left\|\widehat{\mu}-\widehat{\mu}^{*}\right\|_{I}+\left\|\widehat{\mu}^{*}\right\|_{I}\left\|1 / \widehat{f}_{X}-1 / \widehat{f}_{X}^{*}\right\|_{I} \\
& \leq o_{\mathrm{P}}\left(r_{n}\right)+O_{\mathrm{P}}(1)\left\|\widehat{f}_{X}^{*}-\widehat{f}_{X}\right\|_{I}=o_{\mathrm{P}}\left(r_{n}\right) .
\end{aligned}
$$

Now, observe that $\widehat{g}^{*}(x)-g(x)=\frac{1}{\widehat{f}_{X}^{*}(x)} \frac{1}{n h_{n}} \sum_{j=1}^{n}\left\{Y_{j}-g(x)\right\} K_{n}\left(\left(x-W_{j}\right) / h_{n}\right)$. Since $\left\|A_{n}\right\|_{I}=$ $O\left(h_{n}^{\beta+1}\right)=o\left(h_{n} r_{n}\right)$, we have

$$
\widehat{g}^{*}(x)-g(x)=\frac{1}{\widehat{f}_{X}^{*}(x)} \underbrace{\frac{1}{n h_{n}} \sum_{j=1}^{n}\left[\left\{Y_{j}-g(x)\right\} K_{n}\left(\left(x-W_{j}\right) / h_{n}\right)-A_{n}(x)\right]}_{=:(\star)}+o_{\mathrm{P}}\left(r_{n}\right)
$$

uniformly in $x \in I$. Since $(\star)=\widehat{\mu}^{*}(x)-\mathrm{E}\left[\widehat{\mu}^{*}(x)\right]-g(x)\left\{\widehat{f}_{X}^{*}(x)-\mathrm{E}\left[\widehat{f}_{X}^{*}(x)\right]\right\}=O_{\mathrm{P}}\left\{h_{n}^{-\alpha}\left(n h_{n}\right)^{-1 / 2} \sqrt{\log \left(1 / h_{n}\right)}\right\}$ uniformly in $x \in I$, and

$$
\left\|1 / \widehat{f}_{X}^{*}-1 / f_{X}\right\|_{I} \leq O_{\mathrm{P}}(1)\left\|\widehat{f}_{X}^{*}-f_{X}\right\|_{I}=O_{\mathrm{P}}\left\{h_{n}^{-\alpha}\left(n h_{n}\right)^{-1 / 2} \sqrt{\log \left(1 / h_{n}\right)}\right\},
$$

we conclude that

$$
\widehat{g}^{*}(x)-g(x)=\frac{1}{f_{X}(x) n h_{n}} \sum_{j=1}^{n}\left[\left\{Y_{j}-g(x)\right\} K_{n}\left(\left(x-W_{j}\right) / h_{n}\right)-A_{n}(x)\right]+o_{\mathrm{P}}\left(r_{n}\right)
$$

uniformly in $x \in I$. This leads to the desired result of Step 1. Furthermore, the derivation so far yields that

$$
\|\widehat{g}-g\|_{I}=O_{\mathrm{P}}\left\{h_{n}^{-\alpha}\left(n h_{n}\right)^{-1 / 2} \sqrt{\log \left(1 / h_{n}\right)}\right\} .
$$

Step 2. Recall the process $Z_{n}^{*}$ defined in (3). By Step 1 together with the fact that $\inf _{x \in I} s_{n}(x) \gtrsim$ $h_{n}^{-\alpha+1 / 2}$, we have

$$
\widehat{Z}_{n}(x)=f_{X}(x) \sqrt{n} h_{n}(\widehat{g}(x)-g(x)) / s_{n}(x)=Z_{n}^{*}(x)+o_{\mathrm{P}}\left\{\left(\log \left(1 / h_{n}\right)\right)^{-1 / 2}\right\}
$$

uniformly in $x \in I$. Recall the class of functions $\mathcal{F}_{n}^{(2)}$ defined in 14), and consider the empirical process indexed by $\mathcal{F}_{n}^{(2)}: \nu_{n}(f)=\frac{1}{\sqrt{n}} \sum_{j=1}^{n}\left\{f\left(Y_{j}, W_{j}\right)-\mathrm{E}[f(Y, W)]\right\}$ for $f \in \mathcal{F}_{n}^{(2)}$. We apply Theorem 2.1 in Chernozhukov et al. (2016) to approximate $\left\|\nu_{n}\right\|_{\mathcal{F}_{n}^{(2)}}=\left\|Z_{n}^{*}\right\|_{I}$ by the supremum of a Gaussian process. To this end, we shall verify the conditions in Chernozhukov et al. (2016).

First, from the covering number bound for $\mathcal{F}_{n}^{(2)}$ given in Lemma A.6 and finiteness of the second moment of $F_{n}^{(2)}(Y, W)$, there exists a tight Gaussian process $G_{n}$ in $\ell^{\infty}\left(\mathcal{F}_{n}^{(2)}\right)$ with mean zero and the same covariance function as $\left\{\nu_{n}(f): f \in \mathcal{F}_{n}^{(2)}\right\}$. Extend $\nu_{n}$ linearly to $\mathcal{F}_{n}^{(2)} \cup\left(-\mathcal{F}_{n}^{(2)}\right)=\{f,-f$ : $\left.f \in \mathcal{F}_{n}^{(2)}\right\}$, and observe that $\left\|\nu_{n}\right\|_{\mathcal{F}_{n}^{(2)}}=\sup _{f \in \mathcal{F}_{n}^{(2)} \cup\left(-\mathcal{F}_{n}^{(2)}\right)} \nu_{n}(f)$. Note that from Theorem 3.7.28 in Giné and Nickl (2016), $G_{n}$ extends to the linear hull of $\mathcal{F}_{n}^{(2)}$ in such a way that $G_{n}$ has linear sample paths, so that $\left\|G_{n}\right\|_{\mathcal{F}_{n}^{(2)}}=\sup _{f \in \mathcal{F}_{n}^{(2)} \cup\left(-\mathcal{F}_{n}^{(2)}\right)} G_{n}(f)$, and in addition $G_{n}$ has uniformly continuous paths on the symmetric convex hull of $\mathcal{F}^{(2)}$ for the intrinsic $L^{2}$ pseudo-metric. It is not difficult to verify that the covering number of $\mathcal{F}_{n}^{(2)} \cup\left(-\mathcal{F}_{n}^{(2)}\right)$ is at most twice that of $\mathcal{F}_{n}^{(2)}$. In particular, 
$\left\{G_{n}(f): f \in \mathcal{F}_{n}^{(2)} \cup\left(-\mathcal{F}_{n}^{(2)}\right)\right\}$ is a tight Gaussian process in $\ell^{\infty}\left(\mathcal{F}_{n}^{(2)} \cup\left(-\mathcal{F}_{n}^{(2)}\right)\right)$ with mean zero and the same covariance function as $\left\{\nu_{n}(f): f \in \mathcal{F}_{n}^{(2)} \cup\left(-\mathcal{F}_{n}^{(2)}\right)\right\}$.

Next, from Lemma A.4.(iii), $\mathrm{E}\left[\left|Y K_{n}\left((x-W) / h_{n}\right)\right|^{2+\ell}\right] \lesssim h_{n}^{-(2+\ell) \alpha+1}$ for $\ell=0,1,2$, so that $\sup _{f \in \mathcal{F}_{n}^{(2)}} \mathrm{E}\left[|f(Y, W)|^{2+\ell}\right] \lesssim h_{n}^{-\ell / 2}$ for $\ell=0,1,2$. Furthermore, $\mathrm{E}\left[\left|F_{n}^{(2)}(Y, W)\right|^{4}\right] \lesssim h_{n}^{-2}\left(\mathrm{E}\left[Y^{4}\right]+\right.$ $\left.\|g\|_{I}^{4}\right) \lesssim h_{n}^{-2}$. Therefore, applying Theorem 2.1 in Chernozhukov et al. (2016) to $\mathcal{F}_{n}^{(2)} \cup\left(-\mathcal{F}_{n}^{(2)}\right)$ with $B(f) \equiv 0, q=4, A \lesssim 1, v \lesssim 1, b \lesssim h_{n}^{-1 / 2}, \sigma \lesssim 1$ and $\gamma \lesssim 1 / \log n$, yields that there exists a random variable $V_{n}$ having the same distribution as $\left\|G_{n}\right\|_{\mathcal{F}_{n}^{(2)}}$ such that

$$
\left|\left\|\nu_{n}\right\|_{\mathcal{F}_{n}^{(2)}}-V_{n}\right|=O_{\mathrm{P}}\left\{\frac{(\log n)^{5 / 4}}{n^{1 / 4} h_{n}^{1 / 2}}+\frac{\log n}{\left(n h_{n}\right)^{1 / 6}}\right\}=o_{\mathrm{P}}\left\{\left(\log \left(1 / h_{n}\right)\right)^{-1 / 2}\right\} .
$$

Now, for $f_{n, x}(y, w)=\{y-g(x)\} K_{n}\left((x-w) / h_{n}\right) / s_{n}(x)$, define $Z_{n}^{G}(x)=G_{n}\left(f_{n, x}\right)$ for $x \in I$, and observe that $Z_{n}^{G}$ is a tight Gaussian process in $\ell^{\infty}(I)$ with mean zero and the same covariance function as $Z_{n}^{*}$ such that $\left\|Z_{n}^{G}\right\|_{I}$ has the same distribution as $V_{n}$. Since $\left|\left\|\widehat{Z}_{n}\right\|_{I}-V_{n}\right| \leq \mid\left\|\widehat{Z}_{n}\right\|_{I}-$ $\left\|\mathrm{Z}_{n}^{*}\right\|_{I}|+||| \mathrm{Z}_{n}^{*} \|_{I}-V_{n} \mid=o_{\mathrm{P}}\left\{\left(\log \left(1 / h_{n}\right)\right)^{-1 / 2}\right\}$, there exists a sequence $\Delta_{n} \downarrow 0$ such that $\mathrm{P}\left\{\mid\left\|\widehat{Z}_{n}\right\|_{I}-\right.$ $\left.V_{n} \mid>\Delta_{n}\left(\log \left(1 / h_{n}\right)\right)^{-1 / 2}\right\} \leq \Delta_{n}$ (which follows from the fact that the Ky Fan metric metrizes convergence in probability; see Theorem 9.2.2 in Dudley (2002)). Observe that for any $z \in \mathbb{R}$,

$$
\mathrm{P}\left\{\left\|\widehat{Z}_{n}\right\|_{I} \leq z\right\} \leq \mathrm{P}\left\{\left\|Z_{n}^{G}\right\|_{I} \leq z+\Delta_{n}\left(\log \left(1 / h_{n}\right)\right)^{-1 / 2}\right\}+\Delta_{n} .
$$

The anti-concentration inequality for the supremum of a Gaussian process (LemmaA.3) then yields that

$$
\begin{aligned}
& \mathrm{P}\left\{\left\|\mathrm{Z}_{n}^{G}\right\|_{I} \leq z+\Delta_{n}\left(\log \left(1 / h_{n}\right)\right)^{-1 / 2}\right\} \\
& \quad \leq \mathrm{P}\left\{\left\|\mathrm{Z}_{n}^{G}\right\|_{I} \leq z\right\}+4 \Delta_{n}\left(\log \left(1 / h_{n}\right)\right)^{-1 / 2}\left\{1+\mathrm{E}\left[\left\|Z_{n}^{G}\right\|_{I}\right]\right\} .
\end{aligned}
$$

From the covering number bound for $\mathcal{F}_{n}^{(2)}$ given in Lemma A.6. together with the facts that $\mathrm{E}\left[F_{n}^{(2)}(Y, W)^{2}\right] \lesssim h_{n}^{-1}$ and $\operatorname{Var}\left(f_{n, x}(Y, W)\right)=1$ for all $x \in I$, Dudley's entropy integral bound (cf. van der Vaart and Wellner, 1996, Corollary 2.2.8) yields that

$$
\mathrm{E}\left[\left\|Z_{n}^{G}\right\|_{I}\right]=\mathrm{E}\left[\left\|G_{n}\right\|_{\mathcal{F}_{n}^{(2)}}\right] \lesssim \int_{0}^{1} \sqrt{1+\log \left(1 /\left(\delta \sqrt{h_{n}}\right)\right)} d \delta \lesssim \sqrt{\log \left(1 / h_{n}\right)}
$$

which implies that $\mathrm{P}\left\{\left\|\mathrm{Z}_{n}^{G}\right\|_{I} \leq z+\Delta_{n}\left(\log \left(1 / h_{n}\right)\right)^{-1 / 2}\right\} \leq \mathrm{P}\left\{\left\|\mathrm{Z}_{n}^{G}\right\|_{I} \leq z\right\}+o(1)$ uniformly in $z \in \mathbb{R}$. Likewise, we have $\mathrm{P}\left\{\left\|\widehat{Z}_{n}\right\|_{I} \leq z\right\} \geq \mathrm{P}\left\{\left\|Z_{n}^{G}\right\|_{I} \leq z\right\}-o(1)$ uniformly in $z \in \mathbb{R}$. This completes the proof.

A.3. Proof of Theorem 3.2. We first prove the following technical lemma.

Lemma A.8. $\left\|\widehat{s}_{n}^{2}(\cdot) / s_{n}^{2}(\cdot)-1\right\|_{I}=o_{\mathrm{P}}\left\{\left(\log \left(1 / h_{n}\right)\right)^{-1}\right\}$.

Proof. Observe that

$$
\begin{aligned}
& \left\{Y_{j}-\widehat{g}(x)\right\}^{2} \widehat{K}_{n}^{2}\left(\left(x-W_{j}\right) / h_{n}\right)=\left\{Y_{j}-g(x)\right\}^{2} K_{n}^{2}\left(\left(x-W_{j}\right) / h_{n}\right) \\
& \quad+\{g(x)-\widehat{g}(x)\}^{2} K_{n}^{2}\left(\left(x-W_{j}\right) / h_{n}\right) \\
& \quad+2\{g(x)-\widehat{g}(x)\}\left\{Y_{j}-g(x)\right\} K_{n}^{2}\left(\left(x-W_{j}\right) / h_{n}\right) \\
& \quad+\left\{Y_{j}-\widehat{g}(x)\right\}^{2}\left\{\widehat{K}_{n}^{2}\left(\left(x-W_{j}\right) / h_{n}\right)-K_{n}^{2}\left(\left(x-W_{j}\right) / h_{n}\right)\right\},
\end{aligned}
$$


so that

$$
\begin{aligned}
& \left\|\frac{1}{n} \sum_{j=1}^{n}\left\{Y_{j}-\widehat{g}(\cdot)\right\}^{2} \widehat{K}_{n}^{2}\left(\left(\cdot-W_{j}\right) / h_{n}\right)-\frac{1}{n} \sum_{j=1}^{n}\left\{Y_{j}-g(\cdot)\right\}^{2} K_{n}^{2}\left(\left(\cdot-W_{j}\right) / h_{n}\right)\right\|_{I} \\
& \leq O_{\mathrm{P}}\left(h_{n}^{-2 \alpha}\right)\|\widehat{g}-g\|_{I}^{2}+2\|\widehat{g}-g\|_{I}\left\|\frac{1}{n} \sum_{j=1}^{n}\left\{Y_{j}-g(\cdot)\right\} K_{n}^{2}\left(\left(\cdot-W_{j}\right) / h_{n}\right)\right\|_{I} \\
& \quad+\frac{2}{n} \sum_{j=1}^{n}\left(Y_{j}^{2}+\|\widehat{g}\|_{I}^{2}\right)\left\|\widehat{K}_{n}^{2}-K_{n}^{2}\right\|_{\mathbb{R}} .
\end{aligned}
$$

From (17), the first term on the right hand side of $(18)$ is

$$
O_{\mathrm{P}}\left\{h_{n}^{-4 \alpha}\left(n h_{n}\right)^{-1} \log \left(1 / h_{n}\right)\right\} .
$$

Since

$$
\begin{aligned}
\left\|\widehat{K}_{n}-K_{n}\right\|_{\mathbb{R}} & \lesssim \int_{\mathbb{R}}\left|\frac{1}{\widehat{\varphi}_{U}\left(t / h_{n}\right)}-\frac{1}{\varphi_{U}\left(t / h_{n}\right)}\right|\left|\varphi_{K}(t)\right| d t \\
& \leq O_{\mathrm{P}}\left(h_{n}^{-2 \alpha}\right) \int_{\mathbb{R}}\left|\widehat{\varphi}_{U}\left(t / h_{n}\right)-\varphi_{U}\left(t / h_{n}\right)\right|\left|\varphi_{K}(t)\right| d t \\
& =O_{\mathrm{P}}\left(h_{n}^{-2 \alpha} m^{-1 / 2}\right),
\end{aligned}
$$

we have that

$$
\left\|\widehat{K}_{n}^{2}-K_{n}^{2}\right\|_{\mathbb{R}} \leq\left\|\widehat{K}_{n}-K_{n}\right\|_{\mathbb{R}}\left\|\widehat{K}_{n}+K_{n}\right\|_{\mathbb{R}}=O_{\mathrm{P}}\left(h_{n}^{-3 \alpha} m^{-1 / 2}\right),
$$

which implies that the last term on the right hand side on 180 is $O_{\mathrm{P}}\left(h_{n}^{-3 \alpha} m^{-1 / 2}\right)$. To bound the second term, observe first that, since $\mathrm{E}[Y \mid W=w] f_{W}(w)=$ $\left(\left(g f_{X}\right) * f_{U}\right)(w)$ is bounded (in absolute value) by $\left\|g f_{X}\right\|_{\mathbb{R}}$,

$$
\begin{aligned}
& \left\|\mathrm{E}\left[\{Y-g(\cdot)\} K_{n}^{2}\left((\cdot-W) / h_{n}\right)\right]\right\|_{I} \\
& \quad \leq h_{n}\left(\left\|g f_{X}\right\|_{\mathbb{R}}+\|g\|_{I}\left\|f_{W}\right\|_{\mathbb{R}}\right) \int_{\mathbb{R}} K_{n}^{2}(w) d w \lesssim h_{n}^{-2 \alpha+1} .
\end{aligned}
$$

Hence,

$$
\begin{gathered}
\left\|\frac{1}{n} \sum_{j=1}^{n}\left\{Y_{j}-g(\cdot)\right\} K_{n}^{2}\left(\left(\cdot-W_{j}\right) / h_{n}\right)\right\|_{I} \leq \underbrace{\left\|\mathrm{E}\left[\{Y-g(\cdot)\} K_{n}^{2}\left((\cdot-W) / h_{n}\right)\right]\right\|_{I}}_{=O\left(h_{n}^{-2 \alpha+1}\right)} \\
+\left\|\frac{1}{n} \sum_{j=1}^{n}\left\{Y_{j}-g(\cdot)\right\} K_{n}^{2}\left(\left(\cdot-W_{j}\right) / h_{n}\right)-\mathrm{E}\left[\{Y-g(\cdot)\} K_{n}^{2}\left((\cdot-W) / h_{n}\right)\right]\right\|_{I} .
\end{gathered}
$$

The second term on the right hand side is identical to

$$
\left\|\frac{1}{n} \sum_{j=1}^{n}\left\{f\left(Y_{j}, W_{j}\right)-\mathrm{E}[f(Y, W)]\right\}\right\|_{\mathcal{F}_{n}^{(3)}} .
$$


In view of the covering number bound for $\mathcal{F}_{n}^{(3)}$ given in Lemma A.6, together with Theorem 2.14.1 in van der Vaart and Wellner (1996), the expectation of the last term is

$$
\lesssim n^{-1 / 2} \sqrt{\left.\mathrm{E}\left[\left\{F_{n}^{(3)}(Y, W)\right]\right\}^{2}\right]} \lesssim h_{n}^{-2 \alpha} n^{-1 / 2} .
$$

Therefore, the right hand side on $(18)$ is

$$
\begin{aligned}
& O_{\mathrm{P}}\left\{h_{n}^{-4 \alpha}\left(n h_{n}\right)^{-1} \log \left(1 / h_{n}\right)+h_{n}^{-3 \alpha} m^{-1 / 2}\right. \\
& \left.\quad+\left(n h_{n}\right)^{-1 / 2} \sqrt{\log \left(1 / h_{n}\right)}\left(h_{n}^{-2 \alpha+1}+h_{n}^{-2 \alpha} n^{-1 / 2}\right)\right\},
\end{aligned}
$$

which is $o_{\mathrm{P}}\left\{h_{n}^{-2 \alpha+1}\left(\log \left(1 / h_{n}\right)\right)^{-1}\right\}$. Hence, because $\inf _{x \in I} s_{n}^{2}(x) \gtrsim h_{n}^{-2 \alpha+1}$, we have

$$
\begin{aligned}
& \frac{1}{s_{n}^{2}(x) n} \sum_{j=1}^{n}\left\{Y_{j}-\widehat{g}(x)\right\}^{2} \widehat{K}_{n}^{2}\left(\left(x-W_{j}\right) / h_{n}\right) \\
& \quad=\frac{1}{s_{n}^{2}(x) n} \sum_{j=1}^{n}\left\{Y_{j}-g(x)\right\}^{2} K_{n}^{2}\left(\left(x-W_{j}\right) / h_{n}\right)+o_{\mathrm{P}}\left\{\left(\log \left(1 / h_{n}\right)\right)^{-1}\right\}
\end{aligned}
$$

uniformly in $x \in I$. Since $\left\|A_{n}^{2}(\cdot) / s_{n}^{2}(\cdot)\right\|_{I}=O\left(h_{n}^{2 \alpha+2 \beta+1}\right)$, it remains to prove that

$$
\begin{aligned}
& \| \frac{1}{s_{n}^{2}(\cdot) n} \sum_{j=1}^{n}\left\{Y_{j}-g(\cdot)\right\}^{2} K_{n}^{2}\left(\left(\cdot-W_{j}\right) / h_{n}\right) \\
& \left.\quad-\mathrm{E}\left[\frac{1}{s_{n}^{2}(\cdot)}\{Y-g(\cdot)\}^{2} K_{n}^{2}((\cdot-W)) / h_{n}\right)\right] \|_{I} \\
& =\left\|\frac{1}{n} \sum_{j=1}^{n}\left\{f\left(Y_{j}, W_{j}\right)-\mathrm{E}[f(Y, W)]\right\}\right\|_{\mathcal{F}_{n}^{(4)}}
\end{aligned}
$$

is $o_{\mathrm{P}}\left\{\left(\log \left(1 / h_{n}\right)\right)^{-1}\right\}$. In view of the covering number bound for $\mathcal{F}_{n}^{(4)}$ given in Lemma A.6. together with Theorem 2.14.1 in van der Vaart and Wellner (1996), the expectation of the last term is

$$
\lesssim n^{-1 / 2} \sqrt{\left.\mathrm{E}\left[\left\{F_{n}^{(4)}(Y, W)\right]\right\}^{2}\right]} \lesssim h_{n}^{-1} n^{-1 / 2}=o\left\{\left(\log \left(1 / h_{n}\right)\right)^{-1}\right\} .
$$

This completes the proof.

Proof of Theorem 3.2. We divide the proof into several steps.

Step 1. Define

$$
\begin{aligned}
\mathrm{Z}_{n}^{\xi}(x)=\frac{1}{s_{n}(x) \sqrt{n}} \sum_{j=1}^{n} \xi_{j}\left[\left\{Y_{j}\right.\right. & -g(x)\} K_{n}\left(\left(x-W_{j}\right) / h_{n}\right) \\
& \left.\quad-n^{-1} \sum_{j^{\prime}=1}^{n}\left\{Y_{j^{\prime}}-g(x)\right\} K_{n}\left(\left(x-W_{j^{\prime}}\right) / h_{n}\right)\right]
\end{aligned}
$$

for $x \in I$. We first prove that

$$
\sup _{z \in \mathbb{R}}\left|\mathrm{P}\left\{\left\|Z_{n}^{\xi}\right\|_{I} \leq z \mid \mathcal{D}_{n}\right\}-\mathrm{P}\left\{\left\|Z_{n}^{G}\right\|_{I} \leq z\right\}\right| \stackrel{\mathrm{P}}{\rightarrow} 0 .
$$


To this end, we shall apply Theorem 2.2 in Chernozhukov et al. $(2016)$ to $\mathcal{F}_{n}^{(2)} \cup\left(-\mathcal{F}_{n}^{(2)}\right)$. Let

$$
\nu_{n}^{\xi}(f)=\frac{1}{\sqrt{n}} \sum_{j=1}^{n} \xi_{j}\left\{f\left(Y_{j}, W_{j}\right)-n^{-1} \sum_{j^{\prime}=1}^{n} f\left(Y_{j^{\prime}}, W_{j^{\prime}}\right)\right\}, f \in \mathcal{F}_{n}^{(2)} .
$$

Then applying Theorem 2.2 in Chernozhukov et al. (2016) to $\mathcal{F}_{n}^{(2)} \cup\left(-\mathcal{F}_{n}^{(2)}\right)$ with $B(f) \equiv 0, q=$ $4, A \lesssim 1, v \lesssim 1, b \lesssim h_{n}^{-1 / 2}, \sigma \lesssim 1$ and $\gamma \lesssim 1 / \log n$, yields that there exists a random variable $V_{n}^{\xi}$ of which the conditional distribution given $\mathcal{D}_{n}$ is identical to the distribution of $\left\|G_{n}\right\|_{\mathcal{F}_{n}^{(2)}}\left(=\left\|Z_{n}^{G}\right\|_{I}\right)$, and such that

$$
\left|\left\|\nu_{n}^{\xi}\right\|_{\mathcal{F}_{n}^{(2)}}-V_{n}^{\xi}\right|=O_{\mathrm{P}}\left\{\frac{(\log n)^{9 / 4}}{n^{1 / 4} h_{n}^{1 / 2}}+\frac{(\log n)^{2}}{\left(n h_{n}\right)^{1 / 4}}\right\}=o_{\mathrm{P}}\left\{\left(\log \left(1 / h_{n}\right)\right)^{-1 / 2}\right\},
$$

which shows that there exists a sequence $\Delta_{n} \downarrow 0$ such that

$$
\mathrm{P}\left\{\left|\left\|\nu_{n}^{\xi}\right\|_{\mathcal{F}_{n}^{(2)}}-V_{n}^{\xi}\right|>\Delta_{n}\left(\log \left(1 / h_{n}\right)\right)^{-1 / 2} \mid \mathcal{D}_{n}\right\} \stackrel{\mathrm{P}}{\rightarrow} 0 .
$$

Since $\left\|\nu_{n}^{\xi}\right\|_{\mathcal{F}_{n}^{(2)}}=\left\|Z_{n}^{\xi}\right\|_{I}$, we have

$$
\begin{aligned}
\mathrm{P}\left\{\left\|Z_{n}^{\xi}\right\|_{I} \leq z \mid \mathcal{D}_{n}\right\} & \leq \mathrm{P}\left\{V_{n}^{\xi} \leq z+\Delta_{n}\left(\log \left(1 / h_{n}\right)\right)^{-1 / 2} \mid \mathcal{D}_{n}\right\}+o_{\mathrm{P}}(1) \\
& =\mathrm{P}\left\{\left\|Z_{n}^{G}\right\|_{I} \leq z+\Delta_{n}\left(\log \left(1 / h_{n}\right)\right)^{-1 / 2}\right\}+o_{\mathrm{P}}(1)
\end{aligned}
$$

uniformly in $z \in \mathbb{R}$, and the anti-concentration inequality for the supremum of a Gaussian process (Lemma A.3) yields that

$$
\mathrm{P}\left\{\left\|\mathrm{Z}_{n}^{G}\right\|_{I} \leq z+\Delta_{n}\left(\log \left(1 / h_{n}\right)\right)^{-1 / 2}\right\} \leq \mathrm{P}\left\{\left\|Z_{n}^{G}\right\|_{I} \leq z\right\}+o(1)
$$

uniformly in $z \in \mathbb{R}$. Likewise, we have $\mathrm{P}\left\{\left\|\mathrm{Z}_{n}^{\xi}\right\|_{I} \leq z \mid \mathcal{D}_{n}\right\} \geq \mathrm{P}\left\{\left\|\mathrm{Z}_{n}^{G}\right\|_{I} \leq z\right\}-o_{\mathrm{P}}(1)$ uniformly in $z \in \mathbb{R}$.

Step 2. In view of the proof of Step 1, in order to prove the result (10), it is enough to prove that $\left\|\widehat{Z}_{n}^{\xi}-Z_{n}^{\xi}\right\|_{I}=o_{\mathrm{P}}\left\{\left(\log \left(1 / h_{n}\right)\right)^{-1 / 2}\right\}$. To this end, define

$$
\widetilde{Z}_{n}^{\xi}(x)=\frac{1}{s_{n}(x) \sqrt{n}} \sum_{j=1}^{n} \xi_{j}\left\{Y_{j}-\widehat{g}(x)\right\} \widehat{K}_{n}\left(\left(x-W_{j}\right) / h_{n}\right)
$$

for $x \in I$, and we first prove that

$$
\left\|\widetilde{Z}_{n}^{\xi}-Z_{n}^{\xi}\right\|_{I}=o_{\mathrm{P}}\left\{\left(\log \left(1 / h_{n}\right)\right)^{-1 / 2}\right\} .
$$

We begin with noting that

$$
\begin{aligned}
& \frac{1}{n} \sum_{j=1}^{n}\left\{Y_{j}-g(x)\right\} K_{n}\left(\left(x-W_{j}\right) / h_{n}\right) \\
& =h_{n}\left\{\widehat{\mu}^{*}(x)-\mathrm{E}\left[\widehat{\mu}^{*}(x)\right]\right\}-h_{n} g(x)\left\{\widehat{f}_{X}^{*}(x)-\mathrm{E}\left[\widehat{f}_{X}^{*}(x)\right]\right\}+A_{n}(x) \\
& =O_{\mathrm{P}}\left\{h_{n}^{-\alpha+1}\left(n h_{n}\right)^{-1 / 2} \sqrt{\log \left(1 / h_{n}\right)}\right\}
\end{aligned}
$$


uniformly in $x \in I$, so that it suffices to verify that

$$
\begin{gathered}
\| \frac{1}{s_{n}(\cdot) \sqrt{n}}\left[\sum_{j=1}^{n} \xi_{j}\left\{Y_{j}-\widehat{g}(\cdot)\right\} \widehat{K}_{n}\left(\left(\cdot-W_{j}\right) / h_{n}\right)\right. \\
\left.-\sum_{j=1}^{n} \xi_{j}\left\{Y_{j}-g(\cdot)\right\} K_{n}\left(\left(\cdot-W_{j}\right) / h_{n}\right)\right] \|_{I}
\end{gathered}
$$

is $o_{\mathrm{P}}\left\{\left(\log \left(1 / h_{n}\right)\right)^{-1 / 2}\right\}$. Since $\left\|1 / s_{n}\right\|_{I} \lesssim h_{n}^{\alpha-1 / 2}$, the last term is

$$
\begin{aligned}
\lesssim & h_{n}^{\alpha-1 / 2} n^{-1 / 2}\left\{\left\|\sum_{j=1}^{n} \xi_{j} Y_{j}\left\{\widehat{K}_{n}\left(\left(\cdot-W_{j}\right) / h_{n}\right)-K_{n}\left(\left(\cdot-W_{j}\right) / h_{n}\right)\right\}\right\|_{I}\right. \\
& +\|\widehat{g}-g\|_{I}\left\|\sum_{j=1}^{n} \xi_{j} \widehat{K}_{n}\left(\left(\cdot-W_{j}\right) / h_{n}\right)\right\|_{I} \\
& \left.+\|g\|_{I}\left\|\sum_{j=1}^{n} \xi_{j}\left\{\widehat{K}_{n}\left(\left(\cdot-W_{j}\right) / h_{n}\right)-K_{n}\left(\left(x-W_{j}\right) / h_{n}\right)\right\}\right\|_{I}\right\} \\
= & : h_{n}^{\alpha-1 / 2} n^{-1 / 2}\left\{I_{n}+I I_{n}+I I I_{n}\right\} .
\end{aligned}
$$

Step 2 in the proof of Theorem 2 in Kato and Sasaki (2016) shows that $h_{n}^{\alpha-1 / 2} n^{-1 / 2} I I I_{n}=$ $o_{\mathrm{P}}\left\{\left(\log \left(1 / h_{n}\right)\right)^{-1 / 2}\right\}$. For the second term $I I_{n}$, observe that

$$
\begin{aligned}
I I_{n} & \lesssim\|\widehat{g}-g\|_{I} \int_{\mathbb{R}}\left|\sum_{j=1}^{n} \xi_{j} e^{i t W_{j} / h_{n}}\right|\left|\frac{\varphi_{K}(t)}{\widehat{\varphi}_{U}\left(t / h_{n}\right)}\right| d t \\
& \leq O_{\mathrm{P}}\left(h_{n}^{-\alpha}\right)\|\widehat{g}-g\|_{I} \int_{-1}^{1}\left|\sum_{j=1}^{n} \xi_{j} e^{i t Y_{j} / h_{n}}\right| d t \\
& =O_{\mathrm{P}}\left\{h_{n}^{-2 \alpha-1 / 2} \sqrt{\log \left(1 / h_{n}\right)}\right\},
\end{aligned}
$$

so that $h_{n}^{\alpha-1 / 2} n^{-1 / 2} I I_{n}=O_{\mathrm{P}}\left\{h_{n}^{-\alpha-1} n^{-1 / 2} \sqrt{\log \left(1 / h_{n}\right)}\right\}$, and the right hand side is $o_{\mathrm{P}}\left\{\left(\log \left(1 / h_{n}\right)\right)^{-1 / 2}\right\}$ under our assumption. For the first term $I_{n}$, observe that

$$
\begin{aligned}
I_{n} & \lesssim \int_{\mathbb{R}}\left|\sum_{j=1}^{n} \xi_{j} Y_{j} e^{i t W_{j} / h_{n}}\right|\left|\frac{1}{\widehat{\varphi}_{U}\left(t / h_{n}\right)}-\frac{1}{\varphi_{U}\left(t / h_{n}\right)}\right|\left|\varphi_{K}(t)\right| d t \\
& \leq\left\{\int_{-1}^{1}\left|\sum_{j=1}^{n} \xi_{j} Y_{j} e^{i t W_{j} / h_{n}}\right|^{2} d t\right\}\left\{\int_{-1}^{1}\left|\frac{1}{\widehat{\varphi}_{U}\left(t / h_{n}\right)}-\frac{1}{\varphi_{U}\left(t / h_{n}\right)}\right|^{2} d t\right\}^{1 / 2} \\
& =O_{\mathrm{P}}\left(n^{1 / 2} h_{n}^{-2 \alpha} m^{-1 / 2}\right),
\end{aligned}
$$

so that $h_{n}^{\alpha-1 / 2} n^{-1 / 2} I_{n}=o_{\mathrm{P}}\left\{\left(\log \left(1 / h_{n}\right)\right)^{-1}\right\}$. Hence we have proved 19. 
Note that the result of Step 1 and the fact that $\mathrm{E}\left[\left\|\mathrm{Z}_{n}^{G}\right\|_{I}\right]=O\left(\sqrt{\log \left(1 / h_{n}\right)}\right)$ imply that $\left\|\mathrm{Z}_{n}^{\xi}\right\|_{I}=$ $O_{\mathrm{P}}\left(\sqrt{\log \left(1 / h_{n}\right)}\right)$, which in turn implies that $\left\|\widetilde{Z}_{n}^{\xi}\right\|_{I}=O_{\mathrm{P}}\left(\sqrt{\log \left(1 / h_{n}\right)}\right)$. Hence

$$
\left\|\widehat{Z}_{n}^{\xi}-\widetilde{Z}_{n}^{\xi}\right\|_{I} \leq\left\|s_{n}(\cdot) / \widehat{s}_{n}(\cdot)-1\right\|_{I}\left\|\widetilde{Z}_{n}^{\xi}\right\|_{I}=o_{\mathrm{P}}\left\{\left(\log \left(1 / h_{n}\right)\right)^{-1 / 2}\right\}
$$

which leads to 10 .

Step 3. We shall prove the last two assertions of the theorem. Observe that

$$
\begin{aligned}
& \underbrace{\frac{\widehat{f}_{X}(x) \sqrt{n} h_{n}(\widehat{g}(x)-g(x))}{\widehat{s}_{n}(x)}-\widehat{Z}_{n}(x)}_{=: \widehat{Z}_{n}^{\dagger}(x)} \\
& =\left\{\widehat{f}_{X}(x)-f_{X}(x)\right\} \frac{\sqrt{n} h_{n}(\widehat{g}(x)-g(x))}{\widehat{s}_{n}(x)}+\left\{\frac{s_{n}(x)}{\widehat{s}_{n}(x)}-1\right\} \widehat{Z}_{n}(x),
\end{aligned}
$$

and the right hand side is $o_{\mathrm{P}}\left\{\left(\log \left(1 / h_{n}\right)\right)^{-1 / 2}\right\}$ uniformly in $x \in I$. To see this, since $\|\widehat{g}-g\|_{I}=$ $O_{\mathrm{P}}\left\{h_{n}^{-\alpha}\left(n h_{n}\right)^{-1 / 2} \sqrt{\log \left(1 / h_{n}\right)}\right\}$ and $\left\|\widehat{f}_{X}-f_{X}\right\|_{I}=O_{\mathrm{P}}\left\{h_{n}^{-\alpha}\left(n h_{n}\right)^{-1 / 2} \sqrt{\log \left(1 / h_{n}\right)}\right\}$ (which follows from Corollary 1 in Kato and Sasaki (2016)), the right hand side on the above displayed equation is

$$
\begin{aligned}
& O_{\mathrm{P}}\left\{h_{n}^{-\alpha}\left(n h_{n}\right)^{-1 / 2} \sqrt{\log \left(1 / h_{n}\right)}\right\} \times O_{\mathrm{P}}\left(\sqrt{\log \left(1 / h_{n}\right)}\right) \\
& \quad+o_{\mathrm{P}}\left\{\left(\log \left(1 / h_{n}\right)\right)^{-1}\right\} \times O_{\mathrm{P}}\left(\sqrt{\log \left(1 / h_{n}\right)}\right) \\
& =o_{\mathrm{P}}\left\{\left(\log \left(1 / h_{n}\right)\right)^{-1 / 2}\right\}
\end{aligned}
$$

uniformly in $x \in I$. Now, Theorem 3.1 and the anti-concentration inequality for the supremum of a Gaussian process (Lemma A.3) yield that

$$
\sup _{z \in \mathbb{R}}\left|\mathrm{P}\left\{\left\|\widehat{Z}_{n}^{\dagger}\right\|_{I} \leq z\right\}-\mathrm{P}\left\{\left\|\mathrm{Z}_{n}^{G}\right\|_{I} \leq z\right\}\right| \rightarrow 0 .
$$

We are to show that $\mathrm{P}\left\{\left\|\widehat{\mathrm{Z}}_{n}^{\dagger}\right\|_{I} \leq \widehat{c}_{n}(1-\tau)\right\} \rightarrow 1-\tau$. From the result 10$)$, there exists a sequence $\Delta_{n} \downarrow 0$ such that with probability greater than $1-\Delta_{n}$,

$$
\sup _{z \in \mathbb{R}}\left|\mathrm{P}\left\{\left\|\widehat{Z}^{\xi}\right\|_{I} \leq z \mid \mathcal{D}_{n}\right\}-\mathrm{P}\left\{\left\|Z_{n}^{G}\right\|_{I} \leq z\right\}\right| \leq \Delta_{n},
$$

and let $\mathcal{E}_{n}$ be the event that (20) holds. Taking $\Delta_{n} \downarrow 0$ more slowly if necessary, we have that $\sup _{z \in \mathbb{R}}\left|\mathrm{P}\left\{\left\|\widehat{Z}_{n}^{\dagger}\right\|_{I} \leq z\right\}-\mathrm{P}\left\{\left\|Z_{n}^{G}\right\|_{I} \leq z\right\}\right| \leq \Delta_{n}$. Recall that $c_{n}^{G}(1-\tau)$ is the $(1-\tau)$-quantile of $\left\|\mathrm{Z}_{n}^{G}\right\|_{I}$, and observe that on the event $\mathcal{E}_{n}$,

$$
\mathrm{P}\left\{\left\|\widehat{Z}_{n}^{\xi}\right\|_{I} \leq c_{n}^{G}\left(1-\tau+\Delta_{n}\right)\right\} \geq \mathrm{P}\left\{\left\|Z_{n}^{G}\right\|_{I} \leq c_{n}^{G}\left(1-\tau+\Delta_{n}\right)\right\}-\Delta_{n}=1-\tau,
$$

where the last equality holds since the distribution function of $\left\|\mathrm{Z}_{n}^{G}\right\|_{I}$ is continuous (which follows from Lemma A.3. Hence on the event $\mathcal{E}_{n}$, it holds that $\widehat{c}_{n}(1-\tau) \leq c_{n}^{G}\left(1-\tau+\Delta_{n}\right)$, so that

$$
\begin{gathered}
\mathrm{P}\left\{\left\|\widehat{Z}_{n}^{\dagger}\right\|_{I} \leq \widehat{c}_{n}(1-\tau)\right\} \leq \mathrm{P}\left\{\left\|\widehat{Z}_{n}^{\dagger}\right\|_{I} \leq c_{n}^{G}\left(1-\tau+\Delta_{n}\right)\right\}+\Delta_{n} \\
\quad \leq \mathrm{P}\left\{\left\|Z_{n}^{G}\right\|_{I} \leq c_{n}^{G}\left(1-\tau+\Delta_{n}\right)\right\}+2 \Delta_{n}=1-\tau+3 \Delta_{n} .
\end{gathered}
$$

Likewise, we have $\mathrm{P}\left\{\left\|\widehat{Z}_{n}^{\dagger}\right\|_{I} \leq \widehat{c}_{n}(1-\tau)\right\} \geq 1-\tau-3 \Delta_{n}$, which shows that $\mathrm{P}\left\{\left\|\widehat{Z}_{n}^{\dagger}\right\|_{I} \leq \widehat{c}_{n}(1-\tau)\right\} \rightarrow$ $1-\tau$ and thus (11) holds. 
Finally, the Borell-Sudakov-Tsirelson inequality van der Vaart and Wellner, 1996, Lemma A.2.2) yields that

$$
c_{n}^{G}\left(1-\tau+\Delta_{n}\right) \leq \mathrm{E}\left[\left\|Z_{n}^{G}\right\|_{I}\right]+\sqrt{2 \log \left(1 /\left(\tau-\Delta_{n}\right)\right)} \lesssim \sqrt{\log \left(1 / h_{n}\right)},
$$

which implies that $\widehat{c}_{n}(1-\tau)=O_{\mathrm{P}}\left(\sqrt{\log \left(1 / h_{n}\right)}\right)$. Furthermore,

$$
\sup _{x \in I} \widehat{s}_{n}(x) \leq \sup _{x \in I} s_{n}(x) \cdot \sup _{x \in I} \frac{\widehat{s}_{n}(x)}{s_{n}(x)}=O_{\mathrm{P}}\left(h_{n}^{-\alpha+1 / 2}\right) .
$$

Therefore, the supremum width of the band $\widehat{\mathcal{C}}_{1-\tau}$ is

$$
2 \sup _{x \in I} \frac{\widehat{s}_{n}(x)}{\sqrt{n} h_{n}} \widehat{c}_{n}(1-\tau)=O_{\mathrm{P}}\left\{h_{n}^{-\alpha}\left(n h_{n}\right)^{-1 / 2} \sqrt{\log \left(1 / h_{n}\right)}\right\} .
$$

This completes the proof.

A.4. Proof of Theorem 7.1. Let $\widehat{\varphi}_{W Z}(t)=n^{-1} \sum_{j=1}^{n} e^{i\left(t_{1} W_{j}+t_{-1}^{T} Z_{j}\right)}$ and $\widehat{\psi}_{Y W Z}(t)=n^{-1} \sum_{j=1}^{n} Y_{j} e^{i\left(t_{1} W_{j}+t_{-1}^{T} Z_{j}\right)}$. We first point out that $\widehat{\mu}(x, z)$ and $\widehat{f}_{X Z}(x, z)$ can be expressed as

$$
\begin{gathered}
\widehat{\mu}(x, z)=\frac{1}{(2 \pi)^{d+1}} \int_{\mathbb{R}^{d+1}} e^{-i\left(t_{1} x+t_{-1}^{T} z\right)} \widehat{\psi}_{Y W Z}(t) \frac{\varphi_{K}\left(t_{1} h_{n}\right) \varphi_{L}\left(t_{-1} h_{n}\right)}{\widehat{\varphi}_{U}\left(t_{1}\right)} d t \\
\widehat{f}_{X Z}(x, z)=\frac{1}{(2 \pi)^{d+1}} \int_{\mathbb{R}^{d+1}} e^{-i\left(t_{1} x+t_{-1}^{T} z\right)} \widehat{\varphi}_{W Z}(t) \frac{\varphi_{K}\left(t_{1} h_{n}\right) \varphi_{L}\left(t_{-1} h_{n}\right)}{\widehat{\varphi}_{U}\left(t_{1}\right)} d t
\end{gathered}
$$

Given these expressions, the proof of the theorem is almost analogous to the proofs of Theorems 3.1 and 3.2. Essentially we only have to care about the fact the Jacobian of the change of variables $(x, w) \rightarrow\left(h_{n} x, h_{n} z\right)$ is $h_{n}^{d+1}$. For instance, the conclusions of Lemma A.4 change to $\left\|A_{n}\right\|_{I \times J}=O\left(h_{n}^{\beta+d+1}\right), \inf _{(x, z) \in I \times J} s_{n}^{2}(x, z) \gtrsim h_{n}^{-2 \alpha+d+1}$ with $s_{n}^{2}(x, z)=\operatorname{Var}\left(\{Y-g(x, z)\} K_{n}((x-\right.$ $\left.\left.W) / h_{n}\right) L\left((z-Z) / h_{n}\right)\right)$, and $\sup _{(x, z) \in \mathbb{R} \times \mathbb{R}^{d}} \mathrm{E}\left[\left|Y K_{n}\left((x-W) / h_{n}\right) L\left((z-Z) / h_{n}\right)\right|^{2+\ell}\right] \lesssim h_{n}^{-(2+\ell) \alpha+d+1}$ for $\ell=0,1,2$. Let $\widehat{\mu}^{*}(x, z)$ and $\widehat{f}_{X Z}^{*}(x, z)$ be defined by replacing $\widehat{K}_{n}$ in $\widehat{\mu}(x, z)$ and $\widehat{f}_{X Z}(x, z)$, respectively, to $K_{n}$. Then the conclusions of Lemma A.7 change to

$$
\begin{aligned}
& \left\|\widehat{f}_{X Z}^{*}-\mathrm{E}\left[\widehat{f}_{X Z}^{*}\right]\right\|_{\mathbb{R} \times \mathbb{R}^{d}}=O P\left\{h_{n}^{-\alpha}\left(n h_{n}^{d+1}\right)^{-1 / 2} \sqrt{\log \left(1 / h_{n}\right)}\right\}, \\
& \left\|\mathrm{E}\left[\hat{f}_{X Z}^{*}\right]-f_{X Z}\right\|_{\mathbb{R} \times \mathbb{R}^{d}}=O\left(h_{n}^{\beta}\right)=o\left\{h_{n}^{-\alpha}\left(n h_{n}^{d+1} \log \left(1 / h_{n}\right)\right)^{-1 / 2}\right\},
\end{aligned}
$$

and $\left\|\widehat{\mu}^{*}-\mathrm{E}\left[\widehat{\mu}^{*}\right]\right\|_{\mathbb{R} \times \mathbb{R}^{d}}=O_{\mathrm{P}}\left\{h_{n}^{-\alpha}\left(n h_{n}^{d+1}\right)^{-1 / 2} \sqrt{\log \left(1 / h_{n}\right)}\right\}$. Using such estimates, we can show under our assumption that

$\widehat{g}(x, z)-g(x, z)=\frac{1}{f_{X Z}(x, z) n h_{n}^{d+1}} \sum_{j=1}^{n}\left[\left\{Y_{j}-g(x, z)\right\} K_{n}\left(\left(x-W_{j}\right) / h_{n}\right) L\left(\left(z-Z_{j}\right) / h_{n}\right)-A_{n}(x, z)\right]+o_{\mathrm{P}}\left(r_{n}\right)$

with $A_{n}(x, z)=\mathrm{E}\left[\{Y-g(x, z)\} K_{n}\left((x-W) / h_{n}\right) L\left((z-Z) / h_{n}\right)\right]$ and $r_{n}=h_{n}^{-\alpha}\left\{n h_{n}^{d+1} \log \left(1 / h_{n}\right)\right\}^{-1 / 2}$, where $o_{\mathrm{P}}\left(r_{n}\right)$ is uniform in $(x, z) \in I \times J$. Hence, arguing as in Step 2 of the proof of Theorem 3.1 . for the process

$$
\widehat{\mathrm{Z}}_{n}(x, z)=f_{X Z}(x, z) \sqrt{n} h_{n}(\widehat{g}(x, z)-g(x, z)) / s_{n}(x, z)
$$


we obtain the Gaussian approximation: there exists a tight Gaussian process $\left\{Z_{n}^{G}(x, z) ;(x, z) \in\right.$ $I \times J\}$ in $\ell^{\infty}(I \times J)$ with mean zero and covariance function

$\mathrm{E}\left[Z_{n}^{G}(x, z) Z_{n}^{G}(\bar{x}, \bar{z})\right]$

$$
=\frac{\operatorname{Cov}\left(\{Y-g(x, z)\} K_{n}\left((x-W) / h_{n}\right) L\left((z-Z) / h_{n}\right),\{Y-g(\bar{x}, \bar{z})\} K_{n}\left((\bar{x}-W) / h_{n}\right) L\left((\bar{z}-Z) / h_{n}\right)\right)}{s_{n}(x, z) s_{n}(\bar{x}, \bar{z})}
$$

such that

$$
\sup _{y \in \mathbb{R}}\left|\mathrm{P}\left\{\left\|\widehat{Z}_{n}\right\|_{I \times J} \leq y\right\}-\mathrm{P}\left\{\left\|Z_{n}^{G}\right\|_{I \times J} \leq y\right\}\right| \rightarrow 0 .
$$

Likewise, arguing as in the proof of Theorem 3.2 , we can show that

$$
\sup _{y \in \mathbb{R}}\left|\mathrm{P}\left\{\left\|\widehat{Z}_{n}^{\xi}\right\|_{I \times J} \leq y \mid \mathcal{D}_{n}\right\}-\mathrm{P}\left\{\left\|Z_{n}^{G}\right\|_{I \times J} \leq y\right\}\right| \stackrel{\mathrm{P}}{\rightarrow} 0 .
$$

This leads to the validity of the confidence band $\widehat{\mathcal{C}}_{1-\tau}$. The supremum width of the band follows from the fact that $\sup _{(x, z) \in I \times J} \widehat{s}_{n}(x, z)=O_{\mathrm{P}}\left(h_{n}^{-\alpha+(d+1) / 2}\right)$ and the $(1-\tau)$-quantile of $\left\|\mathrm{Z}_{n}^{G}\right\|_{I \times J}$ is $O\left(\sqrt{\log \left(1 / h_{n}\right)}\right)$.

A.5. Proof of Theorem 7.2. The proof is completely analogous to those of Theorems 3.1 and 3.2 , given the facts that $g(y, x)=\mathrm{E}[1(Y \leq y) \mid X=x]$ and the function class $\{1(\cdot \leq y): y \in J\}$ is a VC class. Hence, we omit the details for brevity.

A.6. Validity of the empirical bootstrap confidence band (7). In this section, we show that under the same assumption as Theorem 3.2 , the empirical bootstrap confidence band (7) is asymptotically valid, i.e.,

$$
\mathrm{P}\left\{g(x) \in \widehat{\mathcal{C}}_{1-\tau}^{E B}(x) \forall x \in I\right\}=1-\tau+o(1),
$$

and the supremum width of the band contracts at the rate $O_{\mathrm{P}}\left\{h_{n}^{-\alpha}\left(n h_{n}\right)^{-1 / 2} \sqrt{\log \left(1 / h_{n}\right)}\right\}$. The proof is analogous to that of Theorem 3.2 and so we only point out required modifications. In view of the proof of Theorem 3.2 , it suffices to prove that

$$
\sup _{z \in \mathbb{R}}\left|\mathrm{P}\left\{\left\|\widehat{Z}_{n}^{E B}\right\|_{I} \leq z \mid \mathcal{D}_{n}\right\}-\mathrm{P}\left\{\left\|Z_{n}^{G}\right\|_{I} \leq z\right\}\right| \stackrel{\mathrm{P}}{\rightarrow} 0 .
$$

As in Step 1 of the proof of Theorem 3.2, consider first the infeasible empirical bootstrap process $\mathrm{Z}_{n}^{E B}(x)=\frac{1}{s_{n}(x) \sqrt{n}} \sum_{j=1}^{n}\left[\left\{Y_{j}^{*}-g(x)\right\} K_{n}\left(\left(x-W_{j}^{*}\right) / h_{n}\right)-n^{-1} \sum_{j^{\prime}=1}^{n}\left\{Y_{j^{\prime}}-g(x)\right\} K_{n}\left(\left(x-W_{j^{\prime}}\right) / h_{n}\right)\right]$, which can be rewritten as

$$
\mathrm{Z}_{n}^{E B}(x)=\frac{1}{s_{n}(x) \sqrt{n}} \sum_{j=1}^{n}\left(M_{n j}-1\right)\left\{Y_{j}-g(x)\right\} K_{n}\left(\left(x-W_{j}\right) / h_{n}\right),
$$

where $M_{n j}$ is the number of times that $\left(Y_{j}, W_{j}\right)$ is "redrawn" in the bootstrap sample, and $\left(M_{n 1}, \ldots, M_{n n}\right)$ is multinomially distributed with parameters $n$ and (probabilities) $1 / n, \ldots, 1 / n$ : cf. Chapter 3.6 of van der Vaart and Wellner (1996). Then, instead of Theorem 2.2 in Chernozhukov et al. (2016), we can apply Theorem 2.3 in Chernozhukov et al. (2016) to deduce that

$$
\sup _{z \in \mathbb{R}}\left|\mathrm{P}\left\{\left\|\mathrm{Z}_{n}^{E B}\right\|_{I} \leq z \mid \mathcal{D}_{n}\right\}-\mathrm{P}\left\{\left\|Z_{n}^{G}\right\|_{I} \leq z\right\}\right| \stackrel{\mathrm{P}}{\rightarrow} 0 .
$$


The rest is to verify that $\left\|\widehat{Z}_{n}^{E B}-Z_{n}^{E B}\right\|_{I}=o_{\mathrm{P}}\left\{\left(\log \left(1 / h_{n}\right)\right)^{-1 / 2}\right\}$, but this is quite analogous to Step 2 of the proof of Theorem 3.2 given the expression

$$
\widehat{\mathrm{Z}}_{n}^{E B}(x)=\frac{1}{\widehat{s}_{n}(x) \sqrt{n}} \sum_{j=1}^{n}\left(M_{n j}-1\right)\left\{Y_{j}-g(x)\right\} \widehat{K}_{n}\left(\left(x-W_{j}\right) / h_{n}\right) .
$$

(Recall that $\sum_{j=1}^{n}\left\{Y_{j}-g(x)\right\} \widehat{K}_{n}\left(\left(x-W_{j}\right) / h_{n}\right)=0$.) Hence, we omit the details.

\section{Appendix B. AdDitional simulation Results}

The main text of the paper presents only a subset of simulation results. In this section, we present the remaining simulation results which are not presented in the main text. Table 3 in this appendix show simulation results for (A) $g(x)=x$, (B) $g(x)=x^{2}$, and (C) $g(x)=\cos (x)$. Each table contains results for each of Model 1 and Model 2, for each of the two sample sizes, $n=200$ and 400 , and for each of the two error variance ratios, $1 / 4$ and $1 / 3$. Simulated coverage probabilities are reported for each of the two nominal coverage probabilities, 0.900 and 0.950 .

Figure 4 in this appendix illustrates realizations of estimates and confidence bands for the functions $g(x)=x^{3}$ and $g(x)=\sin (x)$ in Model 2 for error variance ratios of $1 / 4$ and $1 / 3$. Figure 5 in this appendix illustrates realizations of estimates and confidence bands for the functions $g(x)=x$ and $g(x)=x^{2}$ in Model 1 for error variance ratios of $1 / 4$ and $1 / 3$. Similarly, Figure 6 in this appendix illustrates realizations of estimates and confidence bands for the functions $g(x)=x$ and $g(x)=x^{2}$ in Model 2 for error variance ratios of $1 / 4$ and $1 / 3$. Finally, Figure 7 in this appendix illustrates realizations of estimates and confidence bands for the function $g(x)=\cos (x)$ in Model 1 and Model 2 for error variance ratios of $1 / 4$ and $1 / 3$.

\section{Appendix C. Additional Details of Real Data Analysis}

C.1. The background of the empirical study. According to Centers for Disease Control and Prevention (CDC) of the US Department of Health and Human Services, more than one-third $(36.5 \%)$ of US adults have obesity (defined by body mass index or BMI > 30) in the period between 2011 and 2014 (Ogden et al. 2015). The estimated annual medical cost of obesity in the United States was 147 billion 2008 U.S. dollars, with the medical costs for people who are obese being $\$ 1,429$ higher than those of normal weight (Finkelstein et al., 2009). While there is an extensive body of literature on cost estimation of obesity, it is a limitation that commonly used data sets contain only self-reported body measures, and hence the values of BMI generated from them are prone to biases (Bound, et al., 2001). More recently, Cawley and Meyerhoefer (2012) use the instrumental variable approach to address this issue in cost estimation of obesity. In the main text of this paper as well as in the following subsection, we employ our data combination approach to treat the self-reporting errors, and draw confidence bands for nonparametric regressions of medical costs on BMI. We focus on costs measured by medical expenditures. With this said, we note that there are also indirect costs of obesity which we do not account for, e.g., the costs of obesity are

known to be passed on to obese workers with employer-sponsored health insurance in the form of lower cash wages and labor market discrimination against obese job seekers by insurance-providing 


\begin{tabular}{|c|c|c|c|c|c|c|}
\hline \multicolumn{3}{|c|}{ (A) Regression: $g(x)=x$} & \multicolumn{2}{|c|}{$\begin{array}{l}\text { Error Variance } \\
=1 / 4(25 \%)\end{array}$} & \multicolumn{2}{|c|}{$\begin{array}{l}\text { Error Variance } \\
\quad=1 / 3(33 \%)\end{array}$} \\
\hline Model & Probability & Size $(n)$ & Coverage & Length & Coverage & Length \\
\hline \multirow[t]{2}{*}{1} & 0.900 & 200 & 0.898 & 1.996 & 0.899 & 1.887 \\
\hline & & 400 & 0.932 & 1.503 & 0.927 & 1.339 \\
\hline \multirow[t]{2}{*}{1} & 0.950 & 200 & 0.932 & 2.215 & 0.939 & 2.092 \\
\hline & & 400 & 0.967 & 1.661 & 0.955 & 1.489 \\
\hline \multirow[t]{2}{*}{2} & 0.900 & 200 & 0.919 & 1.946 & 0.891 & 1.823 \\
\hline & & 400 & 0.934 & 1.364 & 0.900 & 1.290 \\
\hline \multirow[t]{2}{*}{2} & 0.950 & 200 & 0.951 & 2.156 & 0.929 & 2.021 \\
\hline & & 400 & 0.961 & 1.516 & 0.947 & 1.436 \\
\hline
\end{tabular}

\begin{tabular}{|c|c|c|c|c|c|c|}
\hline \multicolumn{3}{|c|}{ (B) Regression: $g(x)=x^{2}$} & \multirow{2}{*}{\multicolumn{2}{|c|}{$\begin{array}{l}\text { Error Variance } \\
=1 / 4(25 \%)\end{array}$}} & \multirow{2}{*}{\multicolumn{2}{|c|}{$\begin{array}{l}\text { Error Variance } \\
=1 / 3(33 \%)\end{array}$}} \\
\hline \multirow[b]{2}{*}{ Model } & \multirow{2}{*}{$\begin{array}{c}\text { Nominal } \\
\text { Probability }\end{array}$} & \multirow{2}{*}{$\begin{array}{l}\text { Sample } \\
\text { Size }(n)\end{array}$} & & & & \\
\hline & & & Coverage & Length & Coverage & Length \\
\hline \multirow[t]{2}{*}{1} & 0.900 & 200 & 0.881 & 3.601 & 0.897 & 2.950 \\
\hline & & 400 & 0.918 & 3.127 & 0.919 & 2.540 \\
\hline \multirow[t]{2}{*}{1} & 0.950 & 200 & 0.919 & 3.976 & 0.936 & 3.277 \\
\hline & & 400 & 0.954 & 3.464 & 0.954 & 2.828 \\
\hline \multirow[t]{2}{*}{2} & 0.900 & 200 & 0.915 & 3.721 & 0.919 & 3.061 \\
\hline & & 400 & 0.922 & 2.827 & 0.904 & 2.289 \\
\hline \multirow[t]{2}{*}{2} & 0.950 & 200 & 0.947 & 4.134 & 0.946 & 3.406 \\
\hline & & 400 & 0.956 & 3.128 & 0.943 & 2.551 \\
\hline
\end{tabular}

\begin{tabular}{|c|c|c|c|c|c|c|}
\hline \multicolumn{3}{|c|}{ (C) Regression: $g(x)=\cos (x)$} & \multirow{2}{*}{\multicolumn{2}{|c|}{$\begin{array}{l}\text { Error Variance } \\
=1 / 4(25 \%)\end{array}$}} & \multirow{2}{*}{\multicolumn{2}{|c|}{$\begin{array}{l}\text { Error Variance } \\
=1 / 3(33 \%)\end{array}$}} \\
\hline \multirow[b]{2}{*}{ Model } & \multirow{2}{*}{$\begin{array}{c}\text { Nominal } \\
\text { Probability }\end{array}$} & \multirow{2}{*}{$\begin{array}{l}\text { Sample } \\
\text { Size }(n)\end{array}$} & & & & \\
\hline & & & Coverage & Length & Coverage & Length \\
\hline 1 & 0.900 & 200 & 0.882 & 1.588 & 0.891 & 1.470 \\
\hline & & 400 & 0.907 & 1.242 & 0.886 & 1.088 \\
\hline 1 & 0.950 & 200 & 0.926 & 1.768 & 0.936 & 1.631 \\
\hline & & 400 & 0.940 & 1.367 & 0.932 & 1.207 \\
\hline 2 & 0.900 & 200 & 0.901 & 1.587 & 0.888 & 1.451 \\
\hline & & 400 & 0.897 & 1.169 & 0.887 & 1.020 \\
\hline 2 & 0.950 & 200 & 0.945 & 1.765 & 0.944 & 1.612 \\
\hline & & 400 & 0.933 & 1.299 & 0.933 & 1.137 \\
\hline
\end{tabular}

TABLE 3. Simulated uniform coverage probabilities of (A) $g(x)=x$, (B) $g(x)=x^{2}$, and (C) $g(x)=\cos (x)$ by estimated confidence bands in $I=\left[-\sigma_{X}, \sigma_{X}\right]$ under normally distributed $X$ and Laplace distributed $U$. Also reported are the medians of the average band lengths on $I$. The simulated probabilities and lengths are computed for each of the two nominal coverage probabilities, $90 \%$ and $95 \%$, based on 1,000 Monte Carlo iterations. 


$$
n=200
$$

$g(x)=x^{3}$

Model 2

$\mathrm{EV}=1 / 4(25 \%)$

$g(x)=x^{3}$

Model 2

$\mathrm{EV}=1 / 3(33 \%)$
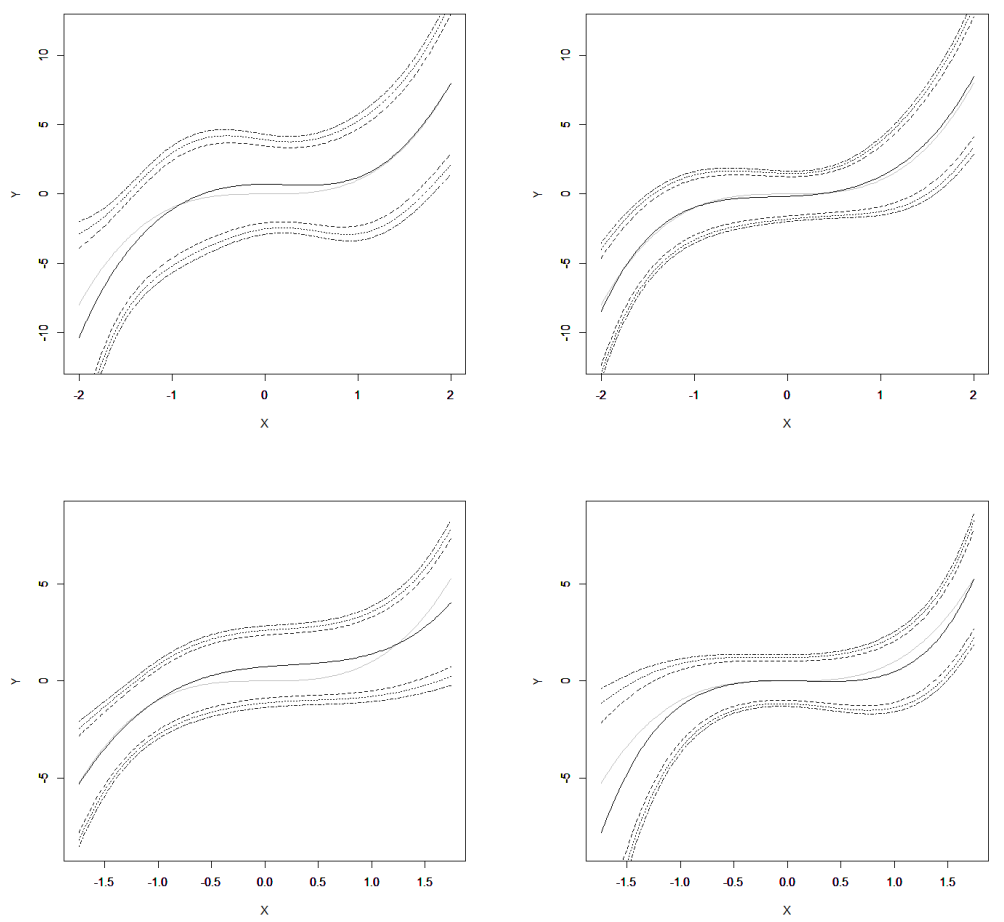

$g(x)=\sin (x)$

Model 2

$\mathrm{EV}=1 / 4(25 \%)$
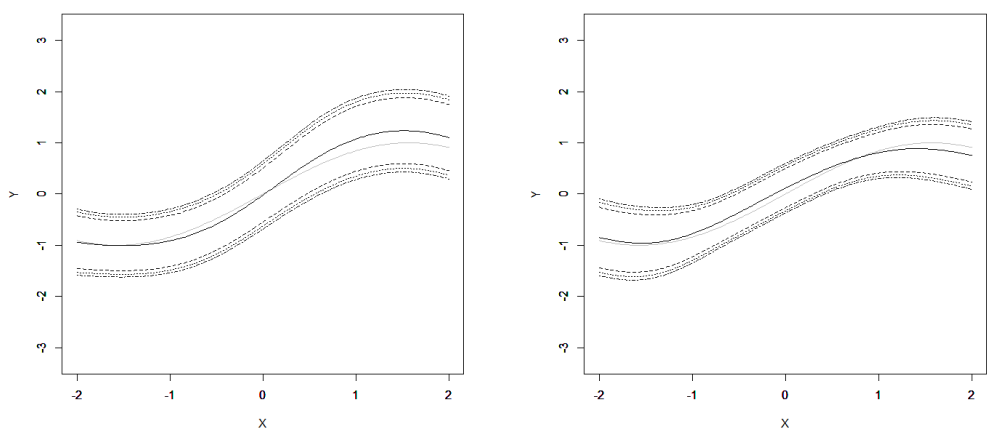

$g(x)=\sin (x)$

Model 2

$\mathrm{EV}=1 / 3(33 \%)$
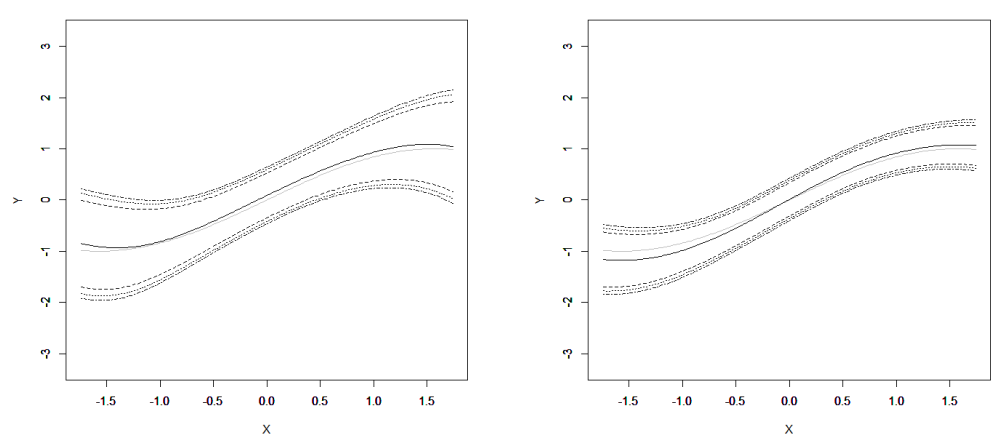

FiguRE 4. Confidence bands for $g(x)=\sin (x)$ and $g(x)=x^{3}$ in Model 2 for error variance ratios of $1 / 4$ and $1 / 3$. Gray curves indicate the true function, black solid curves indicate estimates, and dashed curves indicate the $80 \%, 90 \%$, and $95 \%$ confidence bands. 


$$
n=200
$$

$g(x)=x$

Model 1

$\mathrm{EV}=1 / 4(25 \%)$

$g(x)=x$

Model 1

$\mathrm{EV}=1 / 3(33 \%)$
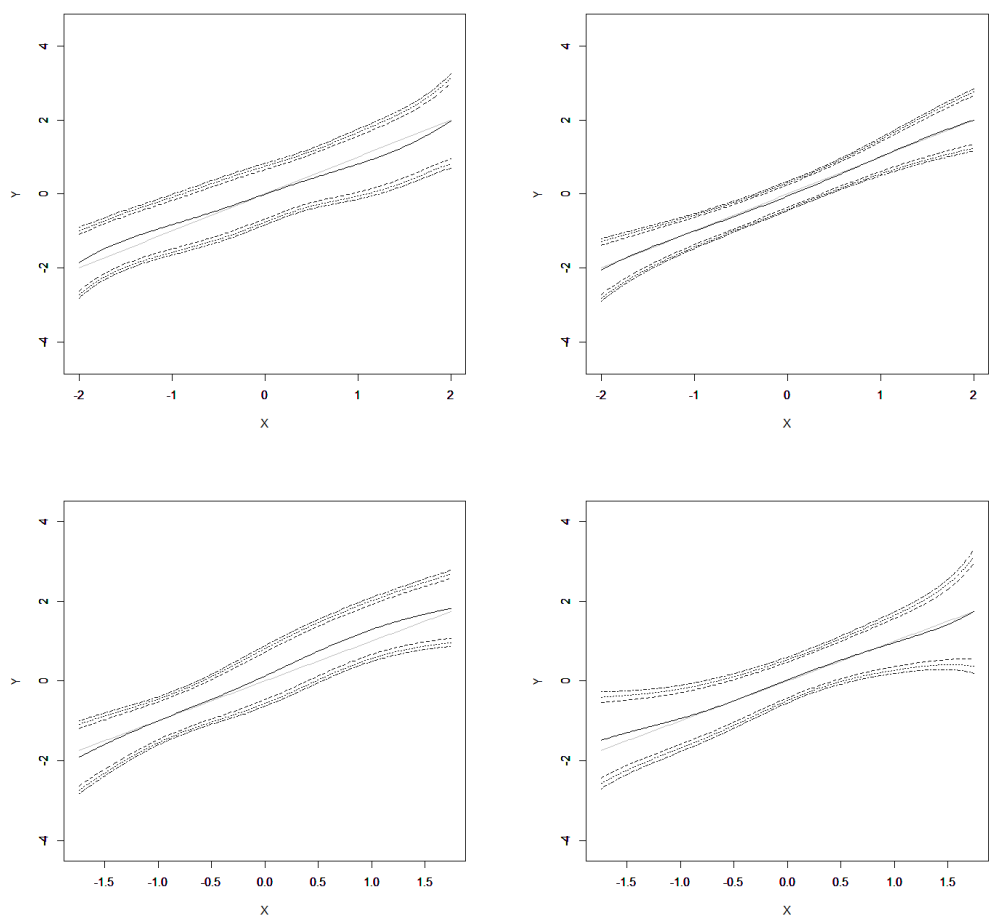

$g(x)=x^{2}$

Model 1

$\mathrm{EV}=1 / 4(25 \%)$
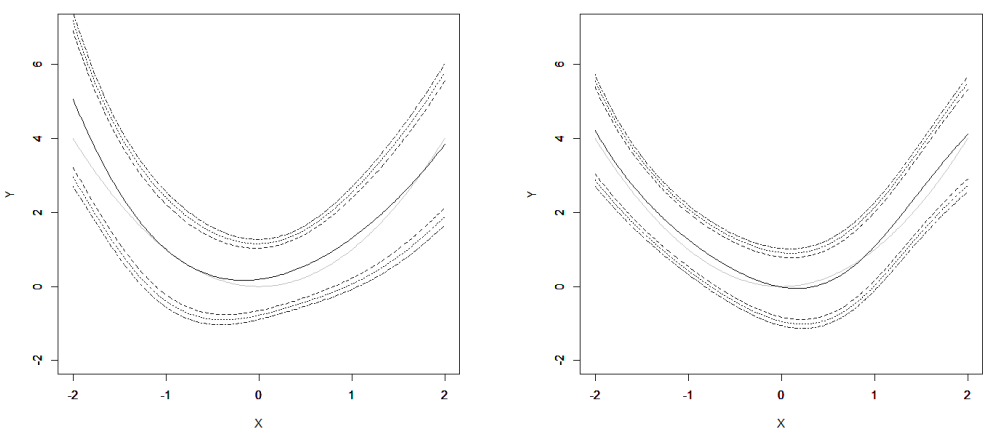

$g(x)=x^{2}$

Model 1

$\mathrm{EV}=1 / 3(33 \%)$
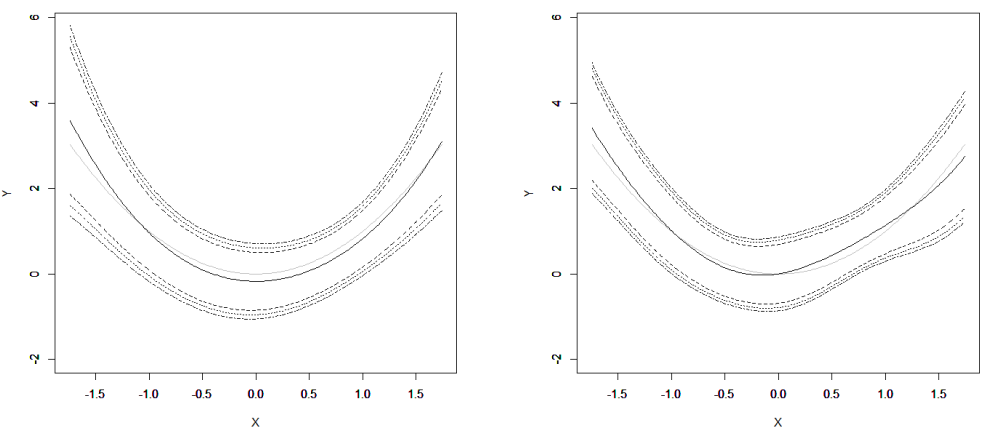

FiguRE 5. Confidence bands for $g(x)=x$ and $g(x)=x^{2}$ in Model 1 for error variance ratios of $1 / 4$ and $1 / 3$. Gray curves indicate the true function, black solid curves indicate estimates, and dashed curves indicate the $80 \%, 90 \%$, and $95 \%$ confidence bands. 


$$
n=200
$$

$g(x)=x$

Model 2

$\mathrm{EV}=1 / 4(25 \%)$

$g(x)=x$

Model 2

$\mathrm{EV}=1 / 3(33 \%)$
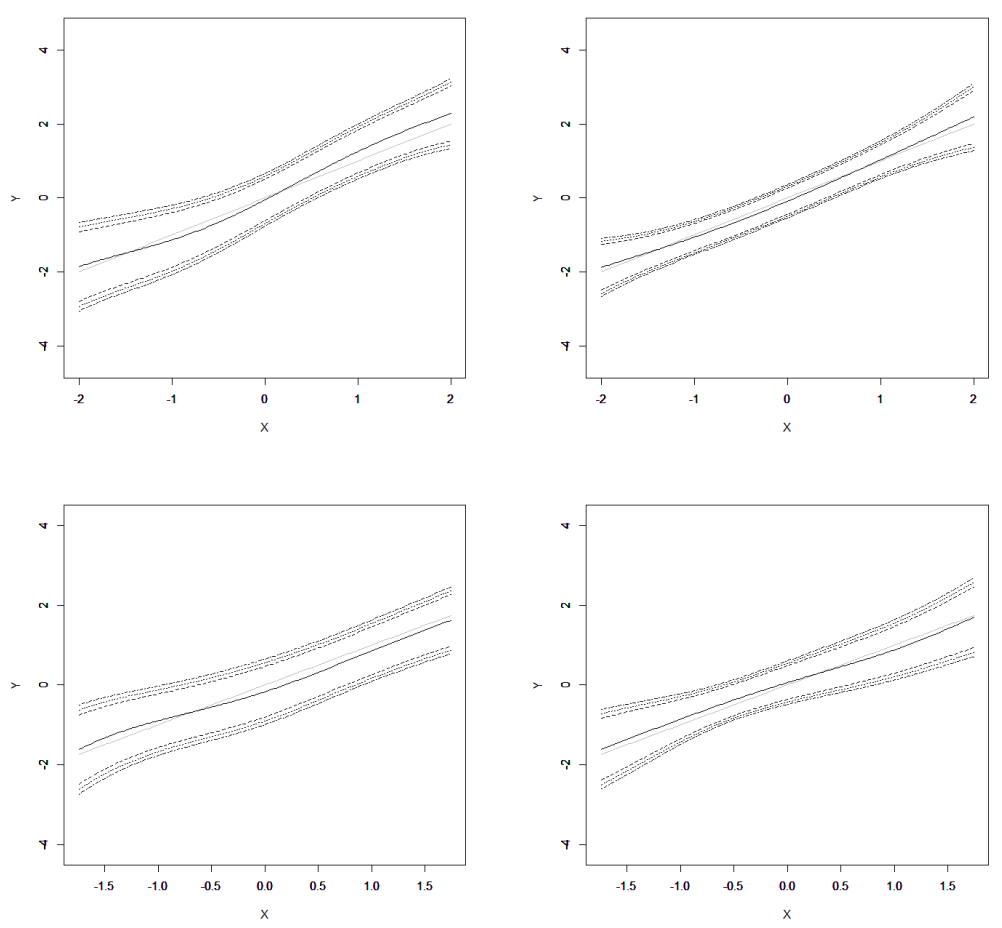

$g(x)=x^{2}$

Model 2

$\mathrm{EV}=1 / 4(25 \%)$
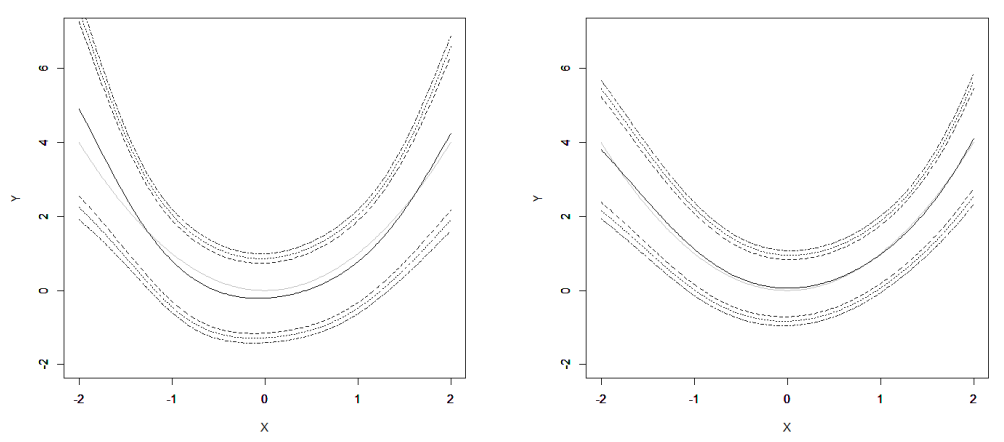

$g(x)=x^{2}$

Model 2

$\mathrm{EV}=1 / 3(33 \%)$
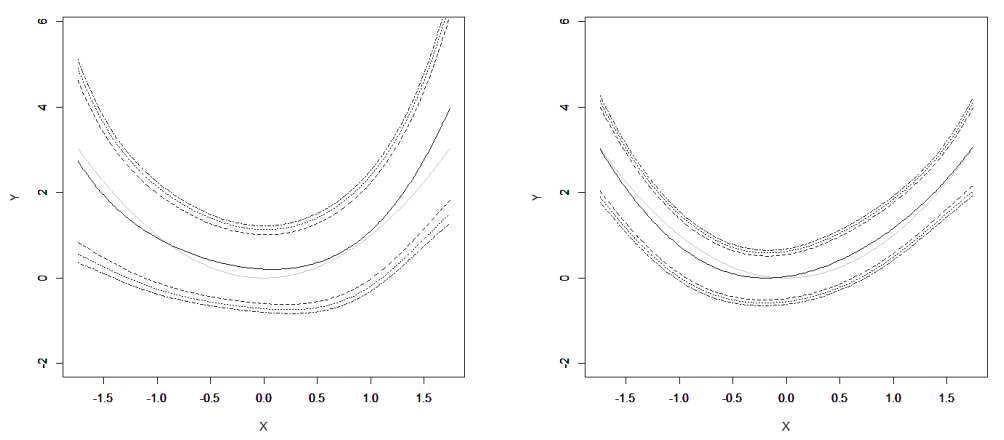

FiguRE 6. Confidence bands for $g(x)=x$ and $g(x)=x^{2}$ in Model 2 for error variance ratios of $1 / 4$ and $1 / 3$. Gray curves indicate the true function, black solid curves indicate estimates, and dashed curves indicate the $80 \%, 90 \%$, and $95 \%$ confidence bands. 


$$
n=200
$$

$g(x)=\cos (x)$

Model 1

$\mathrm{EV}=1 / 4(25 \%)$

$g(x)=\cos (x)$

Model 1

$\mathrm{EV}=1 / 3(33 \%)$
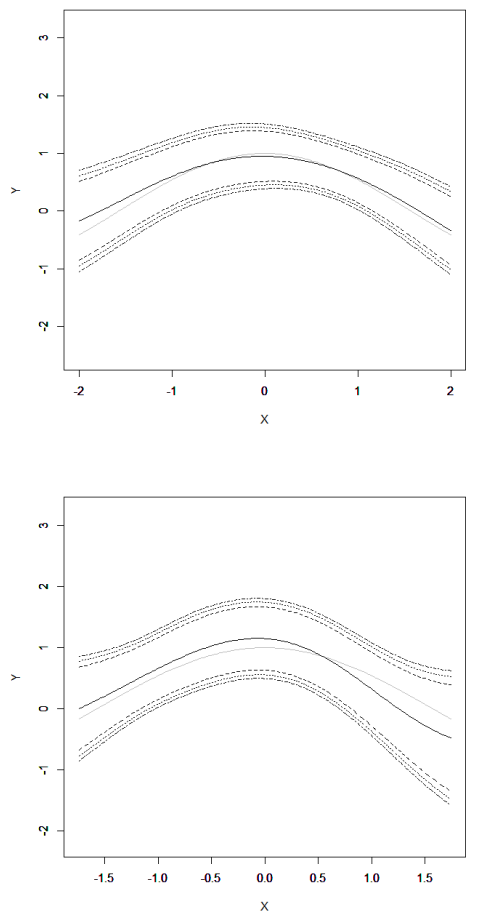

$g(x)=\cos (x)$

Model 2

$\mathrm{EV}=1 / 4(25 \%)$

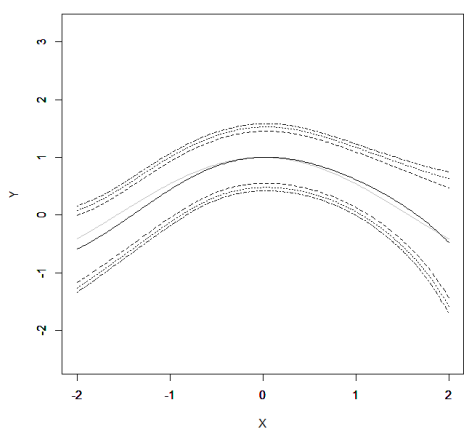

$g(x)=\cos (x)$

Model 2

$\mathrm{EV}=1 / 3(33 \%)$
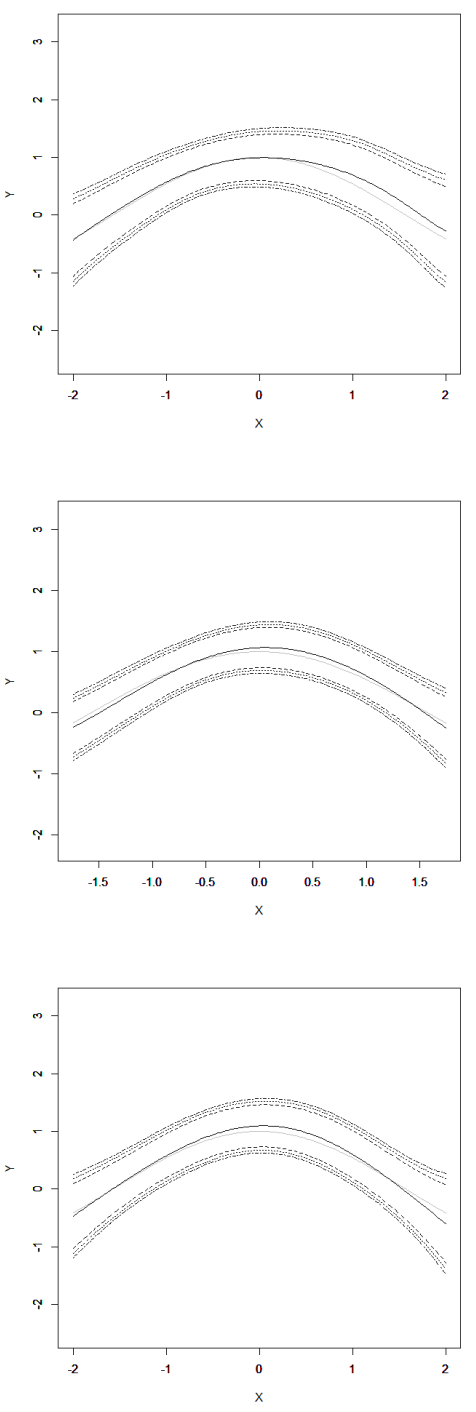

$n=400$

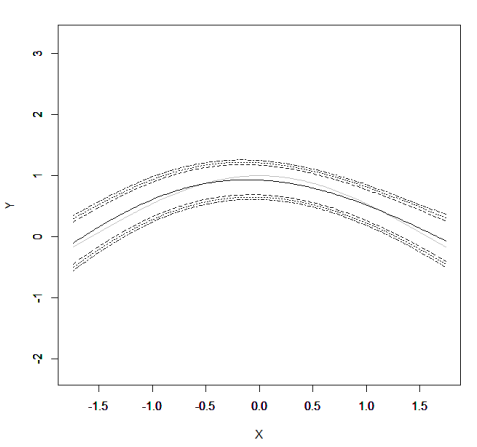

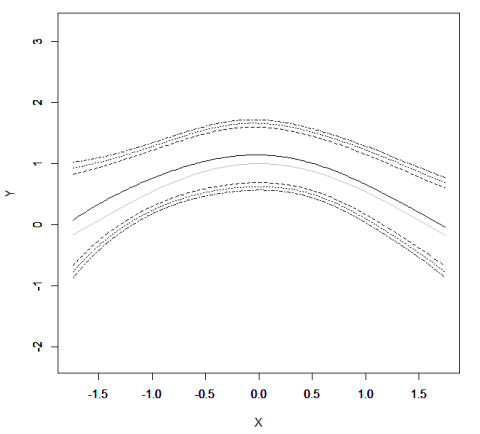

Figure 7. Confidence bands for $g(x)=\cos (x)$ in Model 1 and Model 2 for error variance ratios of $1 / 4$ and $1 / 3$. Gray curves indicate the true function, black solid curves indicate estimates, and dashed curves indicate the $80 \%, 90 \%$, and $95 \%$ confidence bands. 
employers (Bhattacharya and Bundorf, 2009) - see also Cawley $(2004)$. Details of the two data sets which we combine are as follows.

The National Health and Nutrition Examination Survey (NHANES) of CDC contains data of survey responses, medical examination results, and laboratory test results. The survey responses include demographic characteristics, such as gender and age. In addition to the demographic characteristics, the survey responses also contain self-reported body measures and self-reported health conditions. Among the self reported body measures are height in inches and weight in pounds. These two variables allow us to construct the BMI in $\mathrm{lbs} / \mathrm{in}^{2}$ as a generated variable. We convert this unit into the metric unit $\left(\mathrm{kg} / \mathrm{m}^{2}\right)$. The NHANES also contains medical examination results, including clinically measured BMI in $\mathrm{kg} / \mathrm{m}^{2}$. We treat the BMI constructed from the selfreported body measures as $W_{j}$, and the clinically measured BMI as $X_{j}$. From the NHANES as a validation data set of size $m$, we can compute $\eta_{j}=W_{j}-X_{j}$ for each $j=1, \ldots, m$.

The Panel Survey of Income Dynamics (PSID) is a longitudinal panel survey of American families conducted by the Survey Research Center at the University of Michigan. This data set contains a long list of variables including demographic characteristics, socio-economic attributes, expenses, and health conditions, among others. In particular, the PSID contains self-reported body measures of the household head, including height in inches and weight in pounds. These two variables allow us to construct the body mass index (BMI) in $\mathrm{lbs} / \mathrm{in}^{2}$ as a generated variable. Again, we convert this unit into the metric unit $\left(\mathrm{kg} / \mathrm{m}^{2}\right)$. The PSID also contains medical and prescription expenses. We treat the BMI constructed from the self-reported body measures as $W_{j}$, and the medical and prescription expenses as $Y_{j}$. We note that the information contained in the PSID are mostly at the household level, as opposed to the individual level, and thus $Y_{j}$ indicates the total medical and prescription expenses of household $j$. To focus on the individual medical and prescription expenses rather than household expenses, we only consider the sub-sample of the households of single men with no dependent family, for which the total medical and prescription expenses of the household equal to the individual medical and prescription expenses of the household head. Hence, the reported regression results concern these selected subpopulations.

After deleting observations with missing fields from the NHANES 2009-2010, we obtain the following sample sizes of these four subsamples: (a) $m=407$, (b) $m=435$, (c) $m=407$, and (d) $m=431$. After deleting observations with missing fields from the PSID 2009 for prescription expenses as the dependent variable $Y$, we obtain the following sample sizes of these four subsamples: (a) $n=528$, (b) $n=243$, (c) $n=247$, and (d) $n=106$.

C.2. Data sources. The data sets for NHANES are available at:

https://wwwn.cdc.gov/nchs/nhanes/Default.aspx

Clinical measurements are constructed from "Examination" data, while self reports constructed from from "Questionnaire" data. Unique keys are available for combining these two pieces of data sets. The data set for PSID is available at:

\section{https://simba.isr.umich.edu/default.aspx}

We are also happy to provide cleaned data files for immediate use for the sample used for our paper upon request. 
C.3. Additional empirical results. In the main text of the paper, we present empirical results of nonparametric regressions of prescription expenses on BMI. We also ran nonparametric regressions of total medical expenses on BMI. After deleting observations with missing fields from the PSID 2009 for total medical expenses as the dependent variable $Y$, we obtain the following sample sizes of the four subsamples: (a) $n=413$, (b) $n=181$, (c) $n=180$, and (d) $n=64$.

Figure 8 displays estimates and confidence bands for total medical expenses in 2009 US dollars as the dependent variable. The estimates are indicated by solid black curves. The areas shaded by gray-scaled colors indicate $80 \%, 90 \%$, and $95 \%$ confidence bands. The four parts of the figure represent (a) men aged from 20 to 34, (b) men aged from 35 to 49, (c) men aged from 50 to 64, and (d) men aged 65 or above. If we look at the $90 \%$ confidence band for the group (c) of men aged from 50 to 64 , annual average total medical expenses are approximately $\$ 0-\$ 15,561$ if $\mathrm{BMI}=20$, approximately $\$ 1,235-\$ 35,885$ if $\mathrm{BMI}=25$, and approximately $\$ 8,036-\$ 34,465$ if $\mathrm{BMI}=30$.

\section{ACKNOWLEDGMENTS}

We would like to thank Tatsushi Oka and Holger Dette for useful comments and suggestions. We also would like to thank the Editor Yacine Ait-Sahalia, an AE, and two anonymous referees for their constructive comments that helped improve the quality of the paper. 
(a) Men Aged from 20 to 34

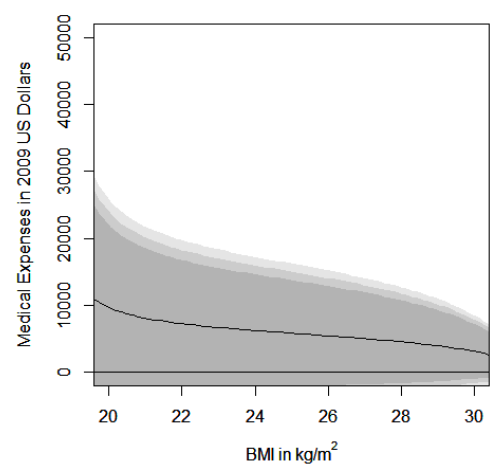

(c) Men Aged from 50 to 64

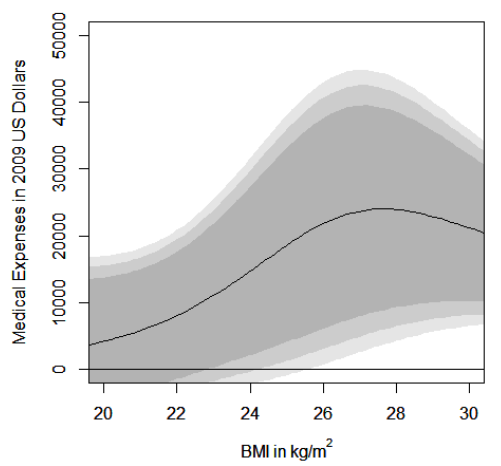

(b) Men Aged from 35 to 49

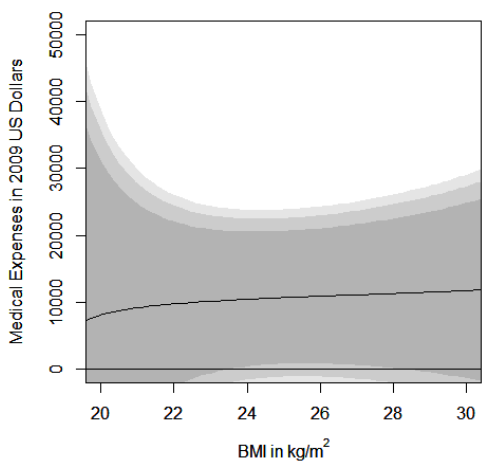

(d) Men Aged 65 or Above

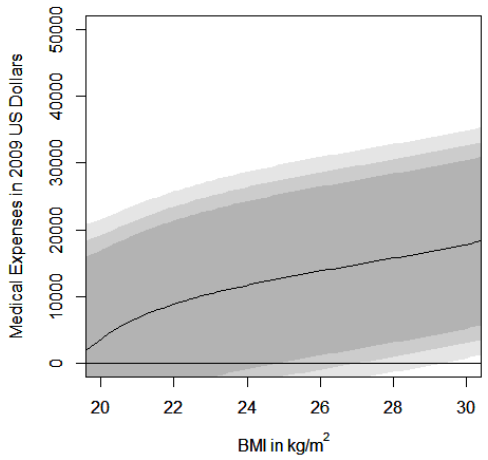

FigURE 8. Estimates and confidence bands for the nonparametric regression of medical expenses on BMI for (a) men aged from 20 to 34, (b) men aged from 35 to 49, (c) men aged from 50 to 64 , and (d) men aged 65 or above. The horizontal axes measure the BMI in $\mathrm{kg} / \mathrm{m}^{2}$. The vertical axes measure the medical expenses in 2009 US dollars. The estimates are indicated by solid black curves. The areas shaded by gray-scaled colors indicate $80 \%$, $90 \%$, and $95 \%$ confidence bands.

\section{REFERENCES}

Adusumilli, K., Otsu, T., and Whang Y.-J. (2016). Inference on distribution functions under measurement error. Preprint.

Armstrong, T. and Kolsár, M. (2018). A simple adjustment for bandwidth snooping. Rev. Econ. Stud. 85 732-765.

Babii, A. (2016). Honest confidence sets in nonparametric IV regression and other ill-posed models. arXiv:1611.03015.

Bhattacharya, J. and Bundorf, M.K. (2009). The incidence of the healthcare costs of obesity. J. Health Econ. 28 649-658. 
Bickel, P. and Rosenblatt, M. (1973). On some global measures of the deviations of density function estimates. Ann. Statist. 1 1071-1095. Correction (1975) 31370.

Birke, M., Bissantz, N., and Holzmann, H. (2010). Confidence bands for inverse regression models. Inverse Problems 26115020.

Bissantz, N., Dümbgen, L., Holzmann, H., and Munk, A. (2007). Non-parametric confidence bands in deconvolution density estimation. J. R. Stat. Soc. Ser. B. Stat. Methodol. 69 483-506.

Bissantz, N. and Holzmann, H. (2008). Statistical inference for inverse problems. Inverse Problems 24034009.

Bohnomme, S. and Robin, J.-M. (2010). Generalized nonparametric deconvolution with an application to earnings dynamics. Rev. Econ. Stud. 77 491-533.

Blundell, R., Chen, X., and Kristensen, D. (2007). Semi-nonparametric IV estimation of shapeinvariant Engel curves. Econometrica 75 1613-1669.

Bound, J., Brown, C., and Mathiowetz, N. (2001). Measurement error in survey data. In: Handbook of Econometrics Vol.5 (eds. J.J. Heckman and E.F. Leamer) Elsevier pp. f3705-3843.

Calonico, S., Cattaneo, M.D., and Farrell, M.H. (2018). On the effect of bias estimation on coverage accuracy in nonparametric inference. J. Amer. Stat. Assoc. 113 763-779.

Card, D., Dobkin, C., and Maestas, N. (2008) The impact of nearly universal insurance coverage on health care utilization: evidence from medicare. Amer. Econ. Rev. 98 2242-2258.

Carroll, R.J. and Hall, P. (1988). Optimal rates of convergence for deconvolving a density. J. Amer. Stat. Assoc. 83 1184-1186.

Carroll, R.J., Maca, J.D., and Ruppert, D. (1999). Nonparametric regression in the presence of measurement error. Biometrika 86 541-554.

Carroll, R.J., Ruppert, D., Stefanski, L.A., and Crainiceanu, C.M. (2006). Measurement Error in Nonlinear Models: A Modern Perspective (2nd Edition). Chapman \& Hall/CRC.

Cavalier, L. (2008). Nonparametric statistical inverse problems. Inverse Problems 24034004.

Cawley, J. (2004). The impact of obesity on wages. J. Hum. Resour. 39 451-474.

Cawley, J. and Meyerhoefer, C. (2012). The medical care costs of obesity: an instrumental variables approach. J. Health Econ. 31 219-230.

Chan, L. K. and Mak, T.K. (1985). On the polynomial functional relationship. J. Roy. Statist. Soc. Ser. B Stat. Methodol. 47 510-518.

Chen, X. and Christensen, T. (2018). Optimal sup-norm rates, adaptivity and inference in nonparametric instrumental variables estimation. Quantitative Economics 9 39-84.

Chen X, Hong, H, and Nekipelov, D. (2011). Nonlinear models of measurement errors. J. Econ. Lit. 49 901-937.

Chen, X., Hong, H., and Tamer, E. (2005). Measurement error models with auxiliary data. Rev. Econ. Stud. 72 343-366.

Chen, X. and Reiss, M. (2011) On rate optimality for ill-posed inverse problems in econometrics. Econometric Theory 27 497-521.

Cheng, C.-L. and Kukush, A.G. (2004) A goodness-of-fit test for a polynomial EIV model. Ukrainian Mathematical Journal 56 641-661. 
Cheng, C.-L. and Schneeweiss, H. (1998). Polynomial regression with errors in the variables. $J$. Roy. Stat. Soc. Ser. B Stat. Methodol. 60 189-199.

Chernozhukov, V., Chetverikov, D., and Kato, K. (2014a). Gaussian approximation of suprema of empirical processes. Ann. Statist. 42 1564-1597.

Chernozhukov, V., Chetverikov, D., and Kato, K. (2014b). Anti-concentration and honest, adaptive confidence bands. Ann. Statist. 42 1787-1818.

Chernozhukov, V., Chetverikov, D., and Kato, K. (2015). Comparison and anti-concentration bounds for maxima of Gaussian random vectors. Probab. Theory Related Fields 162 47-70.

Chernozhukov, V., Chetverikov, D., and Kato, K. (2016). Empirical and multiplier bootstraps for suprema of empirical processes of increasing complexity, and related Gaussian couplings. Stochastic Process. Appl. 126 3632-3651.

Comte, F. and Kappus, J. (2015). Density deconvolution from repeated measurements without symmetry assumption on the errors. J. Multivariate Anal. 140 21-46.

Comte, F. and Lacour, C. (2011). Data-driven density estimation in the presence of additive noise with unknown distribution. J. R. Stat. Soc. Ser. B. Stat. Methodol. 73 601-627.

Delaigle, A., Fan, J., and Carroll, R.J. (2009). A design-adaptive local polynomial estimator for the errors-in-variables problem. J. Amer. Stat. Assoc. 104 348-359.

Delaigle, A. and Hall, P. (2008). Using SIMEX for smoothing-parameter choice in errors-in-variables problems. J. Amer. Stat. Assoc. 103 280-287.

Delaigle, A. and Hall, P. (2016). Methodology for nonparametric deconvolution when the error distribution is unknown. J. R. Stat. Soc. Ser. B. Stat. Methodol. 78 231-252.

Delaigle, A., Hall, P., and Jamshidi, F. (2015). Confidence bands in nonparametric errors-invariables regression. J. R. Stat. Soc. Ser. B. Stat. Methodol. 77 149-169.

Delaigle, A., Hall, P., and Meister, A. (2008). On deconvolution with repeated measurements. Ann. Statist. 36 665-685.

Delaigle, A. and Meister, A. (2007). Nonparametric regression estimation in the heteroscedastic errors-in-variables problem. J. Amer. Stat. Assoc. 102 1416-1426.

Diggle, P.J. and Hall, P. (1993). A Fourier approach to nonparametric deconvolution of a density estimate. J. Roy. Stat. Soc. Ser. B. Stat. Methodol. 55 523-531.

Dudley, R.M. (2002). Real Analysis and Probability. Cambridge University Press.

Efromovich, S. (1997). Density estimation for the case of supersmooth measurement error. J. Amer. Stat. Assoc. 92 526-535.

Einav, L., Leibtag, E., and Nevo, A. (2010). Recording discrepancies in Nielsen Homescan data: Are they present and do they matter? Quant. Mark. Econ. 8 207-239.

van Es, B. and Gugushvili, S. (2008). Weak convergence of the supremum distance for supersmooth kernel deconvolution. Statist. Probab. Lett. 78 2932-2938.

van Es, B. and Uh, H.-W. (2005). Asymptotic normality of kernel-type deconvolution estimators. Scand. J. Statist. 32 467-483.

Eubank, R.L. and Speckman, P.L. (1993). Confidence bands in nonparametric regression. J. Amer. Stat. Assoc. 88 1287-1301. 
Fan, J. (1991a). On the optimal rates of convergence for nonparametric deconvolution problems. Ann. Statist. 19 1257-1272.

Fan, J. (1991b). Asymptotic normality for deconvolution kernel density estimators. Sankhya A 53 97-110.

Fan, J. and Masry, E. (1992). Multivariate regression with errors-in-variables: asymptotic normality for mixing processes. J. Multivariate Anal. 43 237-271.

Fan, J. and Truong, Y.K. (1993). Nonparametric regression with errors in variables. Ann. Statist. 21 1900-1925.

Finkelstein, E.A., Trogdon, J.G., Cohen, J.W., and Dietz, W. (2009). Annual medical spending attributable to obesity: payer- and service-specific estimates. Health Aff. 28 W822-W831.

Folland, G.B. (1999). Real Analysis (2nd Edition). Wiley.

Giné, E. and Nickl, R. (2016). Mathematical Foundations of Infinite-Dimensional Statistical Models. Cambridge University Press.

Hall, P. (1991). On convergence rates of suprema. Probab. Theory Related Fields 89 447-455.

Hall, P. and Horowitz, J.L. (2005). Nonparametric methods for inference in the presence of instrumental variables. Ann. Statist. 33 2904-2929.

Hall, P. and Horowitz, J.L. (2013). A simple bootstrap method for constructing nonparametric confidence bands for functions. Ann. Statist. 41 1892-1921.

Hall, P. and Ma, Y. (2007). Testing the suitability of polynomial models in error-in-variables problems. Ann. Statist. 35 2620-2638.

Hausman, J.A., Newey, W.K., Ichimura, H., and Powell, J.L. (1991). Identification and estimation of polynomial errors-in-variables models. J. Econometrics 50 273-295.

Horowitz, J.L. (2009). Semiparamtric and Nonparametric Methods in Econometrics. Springer.

Horowitz, J.L. (2011). Applied nonparametric instrumental variables estimation. Econometrica 79 347-394.

Horowitz, J. L. and Lee, S. (2012). Uniform confidence bands for functions estimated nonparametrically with instrumental variables. J. Econometrics 168 175-188.

Horowitz, J.L. and Markatou, M. (1996). Semiparametric estimation of regression models for panel data. Rev. Econ. Stud. 63 145-168.

$\mathrm{Hu}, \mathrm{Y}$. and Ridder, G. (2010). On deconvolution as a first stage nonparametric estimator. Econometric Reviews 29 365-396.

$\mathrm{Hu}$, Y. and Sasaki, Y. (2015). Closed-form estimation of nonparametric models with non-classical measurement errors. J. Econometrics 185 392-408.

Johannes, J. (2009). Deconvolution with unknown error distribution. Ann. Statist. 37 2301-2323.

Kato, K. and Sasaki, Y. (2016). Uniform confidence bands in deconvolution with unknown error distribution. arXiv:1608:02251.

Komlós, J., Major, P., and Tusnády, G. (1975). An approximation for partial sums of independent rv's and the sample df I. Z. Warhsch. Verw. Gabiete 32 111-131.

Kotlarski, I. (1967). On characterizing the gamma and the normal distribution. Pac. J. Math. 20 69-76. 
Li, T. (2002). Robust and consistent estimation of nonlinear errors-in-variables models. J. Econometrics 110 1-26.

Li, T. and Vuong, Q. (1998). Nonparametric estimation of the measurement error model using multiple indicators. J. Multivariate Anal. 65 139-165.

Lounici, K. and Nickl, R. (2011). Global uniform risk bounds for wavelet deconvolution estimators. Ann. Statist. 39 201-231.

McMurry, T.L. and Politis, D.N. (2004). Nonparametric regression with infinite order flat-top kernels. J. Nonparametric Statist. 16 549-562.

Meister, A. (2009). Deconvolution Problems in Nonparametric Statistics. Springer.

Neumann, M.H. (1997). On the effect of estimating the error density in nonparametric deconvolution. J. Nonparametric Statist. 7 307-330.

Neumann, M.H. (2007). Deconvolution from panel data with unknown error distribution. J. Multivariate Anal. 98 1955-1968.

Neumann, M.H. and Polzehl, J. (1998). Simultaneous bootstrap confidence bands in nonparametric regression. J. Nonparametric Statist. 9 307-333.

Neumann, M.H. and Reiß, M. (2009). Nonparametric estimation for Lévy processes from lowfrequency observations. Bernoulli 15 223-248.

Newey, W. and Powell, J. (2003). Instrumental variables estimation of non-parametric models. Econometrica 71 1565-1578.

Ogden, C.L., Carroll, M.D., Fryar, C.D., and Flegal, K.M. (2015). Prevalence of obesity among adults and youth: United States, 2011-2014. NCHS Data Brief 219. Hyattsville, MD: National Center for Health Statistics.

Otsu, T. and Taylor, L. (2016). Specification testing for errors-in-variables models. Preprint.

Proksh, K., Bissantz, N., and Dette, H. (2015). Confidence bands for multivariate and time dependent inverse regression models. Bernoulli 21 144-175.

Ridder, G. and Moffitt, R. (2007). The econometrics of data combination. Handbook of Econometrics 6B 5469-5547.

Rio, E. (1994). Local invariance principles and their application to density estimation. Probab. Theory Related Fields 98 21-45.

Robinson, P.M. (1988). Root-n-consistent semiparametric regression. Econometrica 56 931-954.

Schennach, S.M. (2004). Nonparametric regression in the presence of measurement error. Econometric Theory 20 1046-1093.

Schennach, S.M. (2013). Convolution without independence. Cemmap working paper.

Schennach, S.M. (2015). A bias bound approach to nonparametric inference. Cemmap working paper CWP71/15.

Schennach, S.M. (2016). Recent advances in the measurement error literature. In: Annual Review of Economics, Vol. 8, pp. 341-377.

Schennach, S.M. and Hu, Y. (2013). Nonparametric identification and semiparametric estimation of classical measurement error models without side information. J. Amer. Stat. Assoc. 108 177-186.

Schennach, S.M., White H, and Chalak, K. (2012). Local indirect least squares and average marginal effects in nonseparable structural systems. J. Econometrics 166 282-302. 
Schmidt-Hieber, J., Munk, A., and Dümbgen, L. (2013). Multiscale methods for shape constraints in deconvolution: confidence statements for qualitative features. Ann. Statist. 41 1299-1328.

Smirnov, N.V. (1950). On the construction of confidence regions for the density of distribution of random variables. Doklady Akad. Nauk SSSR 74 189-191 (Russian).

Song, W.-X. (2008) Model checking in errors-in-variables regression. J. Multivariate Anal. 99 24062443.

Stefanski, L. and Carroll, R.J. (1990). Deconvoluting kernel density estimators. Statistics 21 169184.

van der Vaart, A.W. and Wellner, J.A. (1996). Weak Convergence and Empirical Processes: With Applications to Statistics. Springer.

Wasserman, L. (2006). All of Nonparamertric Statistics. Springer.

Xia, Y. (1998). Bias-corrected confidence bands in nonparametric regression. J. R. Stat. Soc. Ser. B Stat. Methodol. 60 797-811.

(K. Kato) Department of Statistics and Data Science, Cornell University, 1194 Comstock Hall, IтHACA, NY 14853, USA.

E-mail address: kk976@cornell.edu

(Y. Sasaki) Department of Economics, Vanderbilt University, VU Station B, Box \#351819, 2301 Vanderbilt Place, Nashville, TN 37235, USA.

E-mail address: yuya.sasaki@vanderbilt.edu 